\title{
6d SCFTs, 5d dualities and Tao web diagrams
}

\author{
Hirotaka Hayashi, ${ }^{a, b}$ Sung-Soo Kim, ${ }^{c, e}$ Kimyeong Lee ${ }^{e}$ and Futoshi Yagi ${ }^{d, e}$ \\ ${ }^{a}$ Department of Physics, School of Science, Tokai University, \\ 4-1-1 Kitakaname, Hiratsuka-shi, Kanagawa 259-1292, Japan \\ ${ }^{b}$ Departamento de Física Teórica and Instituto de Física Teórica UAM/CSIC, \\ Universidad Autónoma de Madrid, \\ Cantoblanco, 28049 Madrid, Spain \\ ${ }^{c}$ School of Physics, University of Electronic Science and Technology of China, \\ No. 4, section 2, North Jianshe Road, Chengdu, Sichuan 610054, China \\ ${ }^{d}$ School of Mathematics, Southwest Jiaotong University, \\ West zone, high-tech district, Chengdu, Sichuan 611756, China \\ ${ }^{e}$ Korea Institute for Advanced Study, \\ 85 Hoegi-ro Dongdaemun-gu, Seoul, 02455, Korea \\ E-mail: h.hayashi@tokai.ac.jp, sungsoo.kim@uestc.edu.cn, \\ klee@kias.re.kr, futoshi_yagi@swjtu.edu.cn
}

ABSTRACT: We propose $5 \mathrm{~d}$ descriptions of $6 \mathrm{~d} \mathcal{N}=(1,0)$ superconformal field theories arising from Type IIA brane configurations with an $\mathrm{O}^{-}$-plane. We T-dualize the brane diagram along a compactification circle and obtain a 5-brane web diagram with two $O 7^{-}$planes. The gauge theory description of the resulting $5 \mathrm{~d}$ theory for a given $6 \mathrm{~d}$ superconformal field theory is not unique, and we argue that the non-uniqueness leads to various dual $5 \mathrm{~d}$ gauge theories. There are three sources which lead to the $5 \mathrm{~d}$ dualities. One type comes from either resolving both or one of the two $\mathrm{O}^{-}$-planes. The two situations give us two different ways to read off a $5 \mathrm{~d}$ gauge theory from essentially the same web diagram. The second type originates from different distributions of D5 or D7-branes, shifting the gauge group ranks of the $5 \mathrm{~d}$ quiver theory. The last one comes from the 90 or 45 degree rotations of the 5 -brane web diagram, which is a part of the $\mathrm{SL}(2, \mathbb{Z})$ duality of Type IIB string theory, leading to completely different group structure. These lead to a very rich class of dualities between $5 \mathrm{~d}$ gauge theories whose UV completion is the same $6 \mathrm{~d}$ superconformal field theory. We also explore Higgsing of the $6 \mathrm{~d}$ theories and their $5 \mathrm{~d}$ counterparts. Decoupling the same flavors from the dual $5 \mathrm{~d}$ theories gives rise to another dual $5 \mathrm{~d}$ theories whose UV completion is the same $5 \mathrm{~d}$ superconformal field theory. Finally we propose the $6 \mathrm{~d}$ description of $5 \mathrm{~d}$ theories which is obtained by a generalization of $5 \mathrm{~d} T_{N}$ theories with additional flavors, which turns out not to be in the class of Type IIA brane construction generically.

Keywords: Brane Dynamics in Gauge Theories, Field Theories in Higher Dimensions, Supersymmetry and Duality

ArXiv EPrint: 1509.03300 


\section{Contents}

1 Introduction and summary $\quad 2$

$26 \mathrm{~d} \operatorname{Sp}(N)$ gauge theory with $N_{f}=2 N+8$ and one tensor multiplet 5

$2.15 \mathrm{~d} \mathrm{SU}(N+2)_{0}$ theory with $N_{f}=2 N+8$ flavors $\quad 6$

$2.25 \mathrm{~d} \operatorname{Sp}(N+1)$ theory with $N_{f}=2 N+8$ flavors 9

2.3 Equivalence between $\mathrm{Sp}$ an $\mathrm{SU}$ theories and their flavor decoupling limits $\quad 9$

$\begin{array}{lll}2.4 \text { S-dualities } & 10\end{array}$

$36 \mathrm{~d} \mathrm{SU}(N)$ gauge theory with $N_{f}=N+8$ and $N_{a}=1 \quad 11$

$\begin{array}{lll}3.15 \mathrm{~d} \text { SU } \times \text { SU gauge theory with flavors } & 12\end{array}$

$3.25 \mathrm{~d} \mathrm{SU}(N+1)$ gauge theory with $N_{f}=N+7$ and $N_{a}=1 \quad 14$

$\begin{array}{lll}3.3 & \text { Equivalence of the two 5d descriptions and their flavor decoupling limits } & 15\end{array}$

$\begin{array}{lll}3.4 & \text { S-dualities and mass parameters } & 15\end{array}$

$\begin{array}{ll}3.5 & \text { Distribution duality } \\ 3.6 & \text { Chation }\end{array}$

3.6 Chain of duality and exotic example 20

4 5d dualities from 6d: generalization $\quad 22$

$\begin{array}{lll}4.16 \mathrm{~d} p-\prod \mathrm{SU} \text { quiver theories } & 22\end{array}$

4.1.1 5d SU quviers 24

$\begin{array}{lll}4.1 .2 & 5 \mathrm{~d} \mathrm{Sp}-\prod \mathrm{SU} \text { quivers } & 28\end{array}$

$\begin{array}{lll}4.1 .3 & 5 \mathrm{~d} \text { dualities } & 29\end{array}$

$\begin{array}{ll}\text { 4.1.4 Higgsed cases } & 29\end{array}$

$4.26 \mathrm{~d}$ SU quivers with an antisymmetric hypermultiplet 33

4.2.1 5d SU quivers 34

4.2.2 5d SU quivers with an antisymmetric hypermultiplet 36

$\begin{array}{lll}4.2 .3 & 5 \text { d dualities } & 37\end{array}$

$\begin{array}{lll}\text { 4.2.4 Higgsed cases } & 37\end{array}$

5 Special cases: the $6 \mathrm{~d}$ quiver gauge theories of $\mathrm{Or}^{-}+8 \mathrm{D} 8^{-}$'s configuration 38

$5.16 \mathrm{~d}\left[1_{A}, 8\right]-\mathrm{SU}(N)-\mathrm{SU}(N)-\cdots-[N]$ quiver 39

$5.26 \mathrm{~d}[8]-\mathrm{Sp}(N)-\mathrm{SU}(2 N)-\mathrm{SU}(2 N)-\cdots-\mathrm{SU}(2 N)-[2 N]$ quiver $\quad 45$

$5.36 \mathrm{~d}[8+m]-\mathrm{Sp}(N)_{\mathrm{T}}-\mathrm{SU}(2 N-m)_{\mathrm{T}}-\mathrm{SU}(2 N-2 m)_{\mathrm{T}}-\mathrm{SU}(2 N-3 m)_{\mathrm{T}}-\ldots$

quiver 46

6 6d description of $5 \mathrm{~d} T_{N}$ Tao theory $\quad 46$

$\begin{array}{lll}\text { 6.1 Global symmetry from 7-branes } & 47\end{array}$

$6.25 \mathrm{~d} T_{4}, T_{5}$ and $T_{6}$ Tao theories 49

6.3 Generalization 51

$\begin{array}{lll}7 & \text { Discussions } & 53\end{array}$

A A brief introduction of Tao diagram 


\section{Introduction and summary}

$6 \mathrm{~d} \mathcal{N}=(1,0)$ superconformal field theories (SCFTs) possess many interesting as well as mysterious features. For instance, they may not have an ultraviolet (UV) Lagrangian description. They may have tensionless self-dual strings as fundamental degrees of freedom. Still, they are expected to be well-defined local quantum field theories. Therefore, a better understanding of the 6d SCFTs will deepen our understandings of quantum field theories. Various aspects of $6 \mathrm{~d} \mathcal{N}=(1,0)$ SCFTs have been analyzed recently, for example, the classification from F-theory [1-6], the elliptic genus computation of the self-dual strings [713], the anomaly polynomials [14-20] and also their torus compactifications [21-23] as well as compactifications on other Riemann surfaces [24-26].

Another important way of analyzing a $6 \mathrm{~d} \mathcal{N}=(1,0)$ SCFT is to study a $5 \mathrm{~d} \mathcal{N}=1$ supersymmetric quantum field theory whose UV completion is the $6 \mathrm{~d}$ SCFT. Given a $6 \mathrm{~d}$ SCFT, we first move to a tensor branch where scalars in tensor multiplets acquire a vacuum expectation value (VEV), and then perform a circle compactification with gauge or flavor Wilson lines along the $S^{1}$. After the compactification, we obtain a certain $5 \mathrm{~d} \mathcal{N}=1$ supersymmetric quantum field theory. From this point of view, the Kaluza-Klein (KK) modes become instantons in the $5 \mathrm{~d}$ theory. In other words, the KK modes are dynamically generated in the $5 \mathrm{~d}$ theory, and they become massless at the UV fixed point, namely we recover the $6 \mathrm{~d}$ SCFT at the UV fixed point of the 5 d theory. Although this picture is useful for the analysis of $6 \mathrm{~d}$ SCFTs, it is typically difficult to identify what is a $5 \mathrm{~d}$ description of a $6 \mathrm{~d}$ SCFT on $S^{1}$.

A new way to see a direct connection between a $6 \mathrm{~d}$ SCFT and a $5 \mathrm{~d}$ theory was discovered in [27]. It starts from a brane configuration in Type IIA string theory developed in $[28,29]$, realizing a $6 \mathrm{~d} \operatorname{Sp}(N)$ gauge theory with $N_{f}=2 N+8$ hypermultiplets in the fundamental representation coupled to a tensor multiplet. The fixed point of the $6 \mathrm{~d}$ theory is the $\left(D_{N+4}, D_{N+4}\right)$ minimal conformal matter theory. One then compactifies the $6 \mathrm{~d}$ theory on a circle with Wilson lines and perform T-duality along the circle. Then the brane configuration in Type IIA string theory becomes a 5-brane web in Type IIB string theory. Moreover, it possible to see the 5-brane web diagram yields a $5 \mathrm{~d} \mathrm{SU}(N+2)$ gauge theory with $N_{f}=2 N+8$ hypermultiplets in the fundamental representation. The corresponding web diagram has a particular feature of an infinitely expanding spiral shape. Hence, it is in a class of so-called Tao web diagram introduced in [30], which has been conjectured to give a $5 \mathrm{~d}$ theory whose UV completion is a $6 \mathrm{~d}$ SCFT. This way gives us a direct connection between the $6 \mathrm{~d}\left(D_{N+4}, D_{N+4}\right)$ minimal conformal matter theory and the $5 \mathrm{~d} \mathrm{SU}(N+2)$ gauge theory with $N_{f}=2 N+8$ flavors. $^{1}$

The main aim of this paper is to generalize the connection between the 6d SCFT and the $5 \mathrm{~d}$ theory to a broader class of $6 \mathrm{~d}$ SCFTs realized by a brane configuration in Type IIA string theory constructed in [28, 29]. The system consists of NS5-branes, D6-

\footnotetext{
${ }^{1}$ The same claim that the UV completion of the $5 \mathrm{~d} \mathrm{SU}(N+2)$ gauge theory with $N_{f}=2 N+8$ flavors is the $6 \mathrm{~d}\left(D_{N+4}, D_{N+4}\right)$ minimal conformal matter was also obtained in [31] by the analysis of the instanton operator in the $5 \mathrm{~d}$ theory. The instanton operator analysis is also a useful way to explore $6 \mathrm{~d}$ SCFTs from $5 \mathrm{~d}$ theories [31-34]. The quantization under a one-instanton background can yield instanton states. One may construct a Dynkin diagram of an enhanced flavor symmetry from the instanton states. If the Dynkin diagram is affine, then the $5 \mathrm{~d}$ theory is conjectured to have a $6 \mathrm{~d} \mathrm{UV}$ completion.
} 
branes, D8-branes and an $\mathrm{O}^{-}$-plane. The D6-branes are divided into pieces by NS5-branes, and D8-branes introduce some hypermultiplets in the fundamental representation. The worldvolume theory on the D6-branes yield a $6 \mathrm{~d}$ theory on the tensor branch of a $6 \mathrm{~d}$ SCFT. It is possible to apply the new method [27] to various 6d SCFTs that can be constructed by the brane system with an $\mathrm{O}^{-}$-plane in Type IIA string theory. The $6 \mathrm{~d}$ theories on the tensor branch of the $6 \mathrm{~d}$ SCFTs we will consider are in the following two classes: ${ }^{2}$

$$
6 d \mathrm{Sp}(N)-\mathrm{SU}(2 N+8)-\mathrm{SU}(2 N+16)-\cdots-\mathrm{SU}(2 N+8(n-1))-[2 N+8 n],
$$

and

$$
6 d[1]_{A}-\mathrm{SU}(N)-\mathrm{SU}(N+8)-\mathrm{SU}(N+16)-\cdots-\mathrm{SU}(N+8(n-1))-[N+8 n],
$$

where $n$ is a positive integer and the subscript $A$ stands for the anti-symmetric representation. With the sufficient number of the flavors at the end node of the quiver theories, one may Higgs the theories as in [2]. The renormalization group (RG) flow triggered by the Higgs VEV yields a different 6d theory at low energies. The corresponding gauge theories may have different ranks, and fundamental hypermultiplets will be attached to various nodes, depending on the Higgsing. We will consider such two large families of $6 \mathrm{~d}$ SCFTs realized by Type IIA branes with an $O 8^{-}$-plane. As in [27], we find that a circle compactification of the system followed by T-duality along the $S^{1}$ always yields a Tao web diagram leading to a certain 5d theory which have the $6 \mathrm{~d}$ UV fixed point. Furthermore, the Tao web diagram enables one to read off a gauge theory description of the $5 \mathrm{~d}$ theory.

In the process of going down to $5 \mathrm{~d}$ from $6 \mathrm{~d}$, we find that there are several different choices which yields $5 \mathrm{~d}$ theories with different gauge groups and matter. We, however, claim that those $5 \mathrm{~d}$ theories are dual to each other in the sense that they have the same UV completion as a $6 \mathrm{~d}$ SCFT. We find that there are three different types of the origin for this $5 \mathrm{~d}$ duality.

(i) SU - Sp duality. The first type of the 5 d duality that we argue comes from the choice of whether we resolve two $O 7^{-}$-planes or only one $O 7^{-}$-plane after the T-duality. The starting $6 \mathrm{~d}$ brane setup includes an $O 8^{-}$-plane, and hence the T-duality induces two $O 7^{-}$-planes. An $\mathrm{O7}^{-}$-plane can be split into two 7-branes by the non-perturbative effects in the string coupling [35]. As we have two $O 7^{-}$-planes, we may split the both $O 7^{-}$planes or only one of them into a pair of 7 -branes. ${ }^{3}$ The two descriptions, in fact, express two different $5 \mathrm{~d}$ gauge theories, an $S U$ gauge theory for two resolved $8^{-}$-planes and an $S p$ gauge theory for an $O 8^{-}$-plane, and thus yield different 5-brane web diagrams. On the other hand, since these two web diagrams arise essentially from the same $6 \mathrm{~d}$ brane configuration, the two $5 \mathrm{~d}$ theories are dual to each other with the same $6 \mathrm{~d}$ UV completion. It is also worthy noting that given these dual $5 \mathrm{~d}$ theories which have the same $6 \mathrm{~d}$ UV fixed point, we can decouple flavors of one of the 5 d gauge theories, say $S U$ theories, one by one by sending the masses to $\pm \infty$, leading to the $5 \mathrm{~d}$ theory which has the $5 \mathrm{~d}$ UV fixed point.

\footnotetext{
${ }^{2}$ In our notation, $[k]_{R}$ means $k$ hypermultiplets in the representation $R$. When $R$ is the fundamental representation, we will omit it for simplicity.

${ }^{3}$ Keeping two $\mathrm{O}^{-}$-planes unresolved will not give a $5 \mathrm{~d}$ description due to the explicit existence of the $S^{1}$.
} 
In the same way, we decouple the flavors in the dual $5 \mathrm{~d}$ theories, $S p$ theories, and then the resulting two $5 \mathrm{~d}$ theories should be also dual to each and have the same $5 \mathrm{~d}$ UV fixed point. Hence, we obtain a $5 \mathrm{~d}$ duality between $5 \mathrm{~d} S U$ gauge theories and $S p$ gauge theories with the same flavors in the sense that the dual $5 \mathrm{~d}$ theories have the same $5 \mathrm{~d}$ UV fixed point. In particular, the $5 \mathrm{~d} S U-S p$ duality proposed in [34] which is an example in the class of dual pairs that we consider in this paper.

(ii) Distribution duality. The second type of the 5d duality arises from a choice of how we allocate D5-branes after the T-duality. In the Type IIA brane configuration with an $O 8^{-}$-plane, the fundamental region is either the left side or the right side of the $\mathrm{Or}^{-}$plane since the transverse space to the $\mathrm{O}^{-}$-plane is a real one-dimensional space. After the T-duality, the $\mathrm{O}^{-}$-plane becomes two $\mathrm{O}^{-}$-planes and the transverse space to the $\mathrm{O}^{-}$-planes is a real two-dimensional space. Each $\mathrm{O}^{-}$-plane induces a point-symmetric 5 -brane web configuration with respect to the point where the $O 7^{-}$-plane is located in the two-dimensional plane. Then, the fundamental region is a region bounded by two parallel infinite mirror lines with each line passing through a different $O 7^{-}$-plane. In this case, it is possible to allocate different numbers of D5-branes between the left side and the right side of a line passing through the two $O 7^{-}$-planes by introducing Wilson lines. In other words, along the circumference of the $S^{1}$ where two $O 7^{-}$-planes maximally apart, the position of each D5-brane along this circumference can be distributed by proper Wilson lines. After splitting the two $O 7^{-}$-planes, the different distributions give a different 5 d quiver theory with different ranks of the gauge groups. Again, the different $5 \mathrm{~d}$ theories have the same $6 \mathrm{~d}$ UV fixed point and thus they are dual to each other. It is, in fact, possible for one to show the duality explicitly at the level of the 5-brane web diagrams. For instance, these different distributions can be more naturally understood as certain mass deformations in their S-dual pictures. Again, decoupling flavors in the same way as (i) above yield another 5d dualities in the sense that the $5 \mathrm{~d}$ theories dual to each other have the same $5 \mathrm{~d}$ UV fixed point.

(iii) $\mathbf{S L}(\mathbf{2}, \mathbb{Z})$-duality. The third type of the $5 \mathrm{~d}$ dualities arises from utilizing the $\mathrm{SL}(2, \mathbb{Z})$ duality in Type IIB string theory. The rotation of a 5-brane web in a real twodimensional space called the $(p, q)$ plane is a part of the $\mathrm{SL}(2, \mathbb{Z})$ duality. In particular, S-duality corresponds to the rotation by 90 degrees. Therefore, the 90 degree rotation of any 5-brane webs obtained after the T-duality from the $6 \mathrm{~d}$ brane configuration leads to another dual picture. We also find that there is another dual frame corresponding to a rotation by 45 degrees or the TST-duality. After the 45 degree rotation in the $(p, q)$ plane, the 5-brane web may still admit a 5 d gauge theory description. In that case, this rotation gives another class of dual $5 \mathrm{~d}$ theories.

From the 6d quiver theories of (1.1) and (1.2) and their Higgsed theories, we find that combinations of the above three types of the $5 \mathrm{~d}$ dualities can give rise to various dual pairs of $5 \mathrm{~d}$ theories which have the same $6 \mathrm{~d}$ UV fixed point. Furthermore, it is interesting to note that we will find a sort of "exotic" examples after a chain of the dualities. Namely, those exotic $5 \mathrm{~d}$ theories do not show an enhancement of the flavor symmetry to an affine type when one applies the instanton operator analysis performed in [31]. However, this is not a contradiction since the analysis in [31] only includes instanton states whose total 
instanton number is 1 . Therefore, we argue that the exotic $5 \mathrm{~d}$ theories should also show the enhancement of the flavor symmetry to an affine type when we include instanton states with the higher instanton number.

Among the 6d theories (1.1) and (1.2) with their various Higgsings, we find that it is particularly interesting to focus on a case where the Type IIA brane configuration includes eight D8-branes with an $\mathrm{O}^{-}$-plane on top of each other after the Higgsing. An important point in this case is that the eight D8-branes with the $08^{-}$-plane become two sets of four D7-branes with an $O 7^{-}$-plane after the T-duality. The combination of four D7-branes with an $O 7^{-}$-plane is special in the sense that the S-dual of this combination gives the same 7-brane configuration. Namely, we can still interpret it as four D7-branes and an $\mathrm{OT}^{-}$plane even after the S-duality. Due to this feature, we find that the resulting S-dualized $5 \mathrm{~d}$ quiver may have an $S U$ gauge node with an anti-symmetric hypermultiplet or an $S p$ gauge node at either or both ends of the quiver, depending on the splitting type of an $\mathrm{Or}^{-}$-plane. Namely, these special $6 \mathrm{~d}$ theories yield yet another class of dual $5 \mathrm{~d}$ theories.

As described above, we can relate various brane configurations in Type IIA string theory to Tao web diagrams in Type IIB string theory. We also note that there exists another class of Tao web diagrams that do not arise from the T-duality from a brane system in Type IIA string theory. The $5 \mathrm{~d}$ theories from that class of the Tao web diagrams can be obtained by adding some flavors at the end nodes of the $5 \mathrm{~d}$ linear quiver theory realizing the $5 \mathrm{~d} T_{N}$ theory [30]. We will call the $5 \mathrm{~d}$ theory as $5 \mathrm{~d}$ " $T_{N}$ Tao" theory. Although the Tao web diagram for a general $5 \mathrm{~d} T_{N}$ Tao theory may not have a direct connection to a Type IIA brane system, we will propose a $6 \mathrm{~d}$ description of the $5 \mathrm{~d} T_{N}$ Tao theory by matching the global symmetry as well as the number of certain multiplets between the two theories.

The organization of this paper is as follows. In section 2, we first start the discussion of the $5 \mathrm{~d}$ dualities that arise from the simplest $6 \mathrm{~d}$ setup corresponding to (1.1) with $n=1$. We then move onto the next simplest example that is (1.2) with $n=1$ in section 3 . In this case, we will see the appearance of the three types of the $5 \mathrm{~d}$ dualities as well as an exotic example. In section 4, we extend our analysis to $5 \mathrm{~d}$ dualities for general $6 \mathrm{~d}$ quiver theories of (1.1) and (1.2) and also the ones after various Higgsings. In section 5, we focus on a special case where the Type IIA brane configuration has eight D8-branes and an $O 8^{-}$-plane on top of each other. In this case, we find that the dual $5 \mathrm{~d}$ theories can become another class that has not been obtained in the other sections. In section 6 , we propose a $6 \mathrm{~d}$ description of the $5 \mathrm{~d} T_{N}$ Tao theory and we find that it is not generically written by a Type IIA brane setup. In appendix A we give a brief introduction of Tao web diagrams.

While we are writing this paper, a related work [36] has appeared recently.

\section{$26 \mathrm{Sp}(N)$ gauge theory with $N_{f}=2 N+8$ and one tensor multiplet}

Various brane constructions in Type IIA string theory for $6 \mathrm{~d}$ anomaly free theories were done in $[28,29]$ in terms of an O8 orientifold, D8-branes, D6-branes, and NS5-branes. In this section, we analyze a circle compactification of a $6 \mathrm{~d} \operatorname{Sp}(N)$ theory with $2 N+8$ hypermultiplets in the fundamental representation and a tensor multiplet whose brane configuration has an $\mathrm{Or}^{-}$orientifold. The T-duality along the $S^{1}$ can give a $5 \mathrm{~d} \mathrm{SU}(N+2)$ 

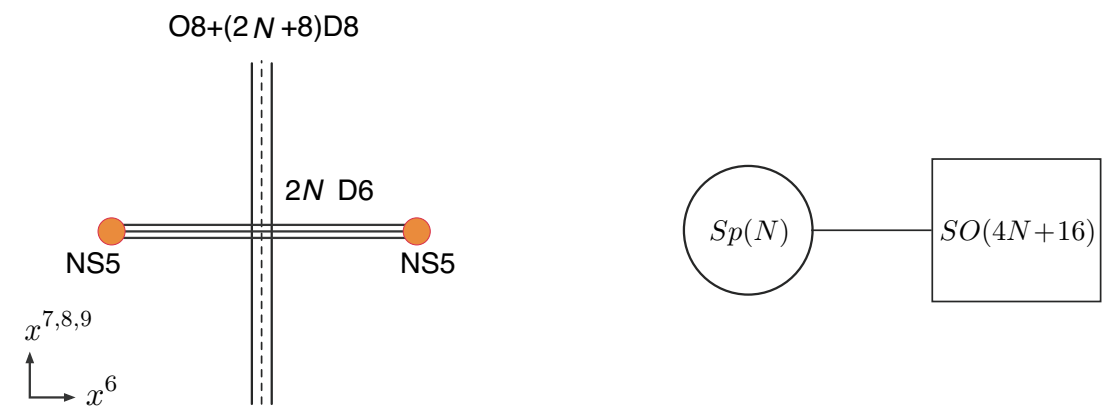

Figure 1. Left: type IIA brane realization of the $\left(D_{N+4}, D_{N+4}\right)$ minimal conformal matter in the tensor branch. Right: the quiver diagram of the $6 \mathrm{~d}$ theory.

with $2 N+8$ flavors as shown in [27], However, we claim that the same brane configuration also yields a $5 \mathrm{~d} S p$ gauge theory with the same rank and the same number of flavors. There was an interesting observation [34] that the superconformal indices for seemingly different 6d theories are equivalent: one is $5 \mathrm{~d} \mathrm{SU}(N+2)$ gauge theory with $N_{f}$ flavors and the Chern-Simons (CS) level $\kappa=N+4-\frac{N_{f}}{2}$, and the other is $5 \mathrm{~d} \operatorname{Sp}(N+1)$ gauge theory with $N_{f}$ flavors. Thus, it implies that the two theories are dual to each other. We here attempt to give an account for this equivalence from the perspective of different pattern of the resolution of $\mathrm{O}^{-}$orientifold planes in Type IIB string theory. As the decoupling limit of flavors yields another 5d theories of 5d UV fixed point, such a dual picture still holds for resulting $5 \mathrm{~d}$ theories with less flavors if one decouples exactly the same flavors in the both pictures. Depicted in the $(p, q)$ 5-brane web diagram, the resulting $5 \mathrm{~d}$ theories also have an S-dual picture.

Consider the $6 \mathrm{~d} \mathcal{N}=(1,0) \operatorname{Sp}(N)$ gauge theory with $N_{f}=2 N+8$ flavors and one tensor multiplet. The UV fixed point for this theory is known as $6 \mathrm{~d}\left(D_{N+4}, D_{N+4}\right)$ minimal conformal matter theory. We compactify this theory on a circle to obtain $5 \mathrm{~d}$ theories. Depending on the choice of Wilson lines, we either get $5 \mathrm{~d} \mathrm{SU}(N+2)_{0}$ theory with $N_{f}=$ $2 N+8$ flavors and the zero CS level, ${ }^{4}$ or $5 \mathrm{~d} \operatorname{Sp}(N+1)$ theory with the same number of flavors. Although the introduction of the Wilson line breaks the global symmetry, in the symmetric phase, both have the same global symmetry as that of its $6 \mathrm{~d}$ "mother" theory, $\mathrm{SO}(4 N+16)$. We note that, as discussed in [27], the $5 \mathrm{~d}$ global symmetry has an additional $\mathrm{U}(1)_{I}$ factor associated to the KK modes. We discuss the two 5 d theories using $(p, q)$ 5-brane web diagrams in details.

\section{$2.15 \mathrm{~d} \mathrm{SU}(N+2)_{0}$ theory with $N_{f}=2 N+8$ flavors}

In Type IIA description, $6 \mathrm{~d}$ brane configuration $[28,29]$ for $\mathcal{N}=(1,0) \operatorname{Sp}(N)$ gauge theory with $N_{f}=2 N+8$ flavors and a tensor multiplet consists of $2 N$ D6 branes (its mirror images included) suspended between a NS5 brane and its mirror NS5 brane through an orientifold plane $O 8^{-}$together with $(2 N+8)$ D8 branes on top of $O 8^{-}$, as depicted in

\footnotetext{
${ }^{4}$ From here on, we use the following short hand notation for denoting the CS level of the SU $(n)$ theory: $\mathrm{SU}(n)_{\kappa}$ is an $\mathrm{SU}(n)$ theory of the CS level $\kappa$.
} 


\begin{tabular}{|c|llllll|l|lll|}
\hline & 0 & 1 & 2 & 3 & 4 & 5 & 6 & 7 & 8 & 9 \\
\hline D6-brane & $\times$ & $\times$ & $\times$ & $\times$ & $\times$ & $\times$ & $\times$ & & \\
NS5-brane & $\times$ & $\times$ & $\times$ & $\times$ & $\times$ & $\times$ & & & \\
D8-brane & $\times$ & $\times$ & $\times$ & $\times$ & $\times$ & $\times$ & & $\times$ & $\times$ & $\times$ \\
O8-plane & $\times$ & $\times$ & $\times$ & $\times$ & $\times$ & $\times$ & & $\times$ & $\times$ & $\times$ \\
\hline
\end{tabular}

Table 1. The brane configuration that yields a $6 \mathrm{~d}$ theory on a tensor branch of a $6 \mathrm{~d}$ SCFT in Type IIA string theory.
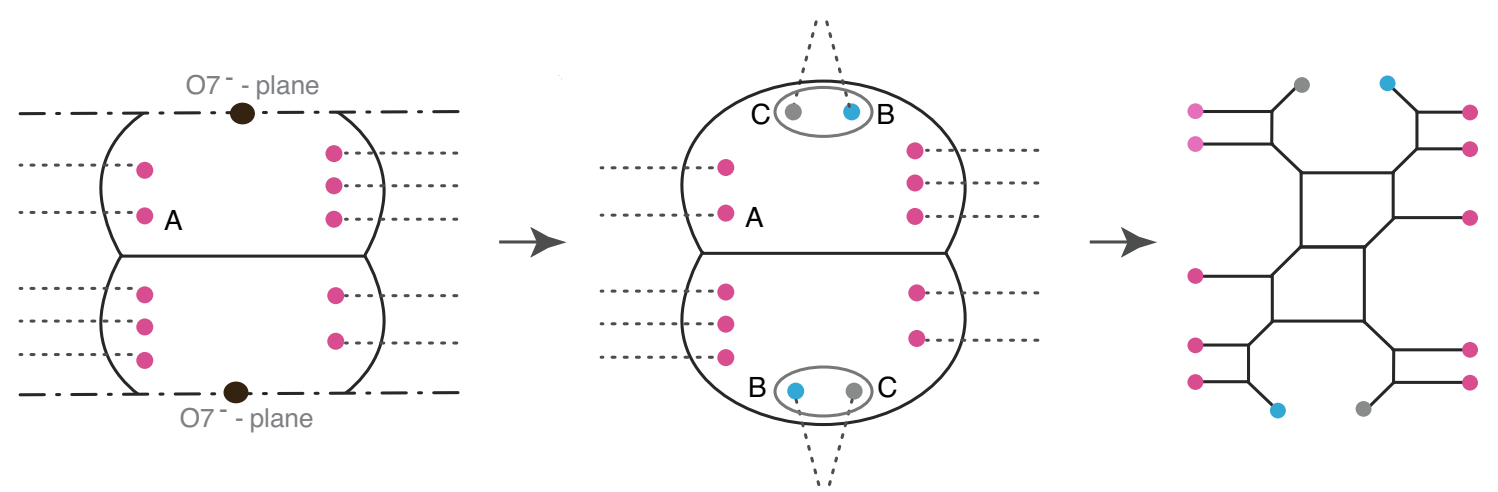

Figure 2. Type IIB brane descriptions for $6 \mathrm{~d} \mathcal{N}=(1,0) \operatorname{Sp}(N)$ gauge theory with one tensor multiplet and $2 N+8$ flavors in the fundamental representation, which yields $\mathcal{N}=1 \mathrm{SU}(N+2)$ gauge theory with the number of flavors. The horizontal direction is $x^{6}$ and the vertical direction is the T-dualized direction. For simplicity, $N=1$. Left: the brane configuration with two $\mathrm{O}^{-}$ planes. A dashed line denotes the branch cut of 7-brane, while a dash-dot line denotes the mirror reflection cut of an $O 7^{-}$plane. Middle: the brane configuration with two $O 7^{-}$planes which are non-perturbatively resolved. Here we denote $\mathbf{A}$ for a D7 brane, $\mathbf{B}$ for a $[1,-1]$ 7-brane, and $\mathbf{C}$ for a $[1,1]$ 7-brane. Right: pulling out 7-branes gives rise to a web configuration for $\mathrm{SU}(3)$ gauge theory with $N_{f}=10$ flavors and zero CS level.

figure 1. The brane configuration in the ten-dimensional spacetime in Type IIA string theory is summarized in table 1.

To have the $5 \mathrm{~d}$ picture, we compactify one of the worldvolume direction of the NS5brane that are parallel to the worldvolume of D6-branes, for example $x^{5}$, on $S^{1}$ with a Wilson line, and then take T-dual along the compactified direction. The resulting 5-brane configuration involves $N$ D5-branes, two NS5-branes, the $(2 N+8)$ D7-branes and two $O 7^{-}$-planes which are maximally separated apart along the T-dualized circle. The Wilson line putting $N$ D5-branes away from the $O 7^{-}$-planes may break the $6 \mathrm{~d} \operatorname{Sp}(N)$ gauge group to $5 \mathrm{~d} \mathrm{U}(N)$. The non-perturbative effect in string theory can resolve the $O 7^{-}$-planes into a pair of two $[p, q]$ 7-branes subject to the monodromy condition that the monodromy of the $O 7^{-}$-plane with four D7-branes is the minus of the identity [35]. For instance, $\mathrm{O}^{-}$can be resolved into a pair of $[1,1]$ and $[1,-1] 7$-branes. As one resolves the $O 7^{-}$planes, the 

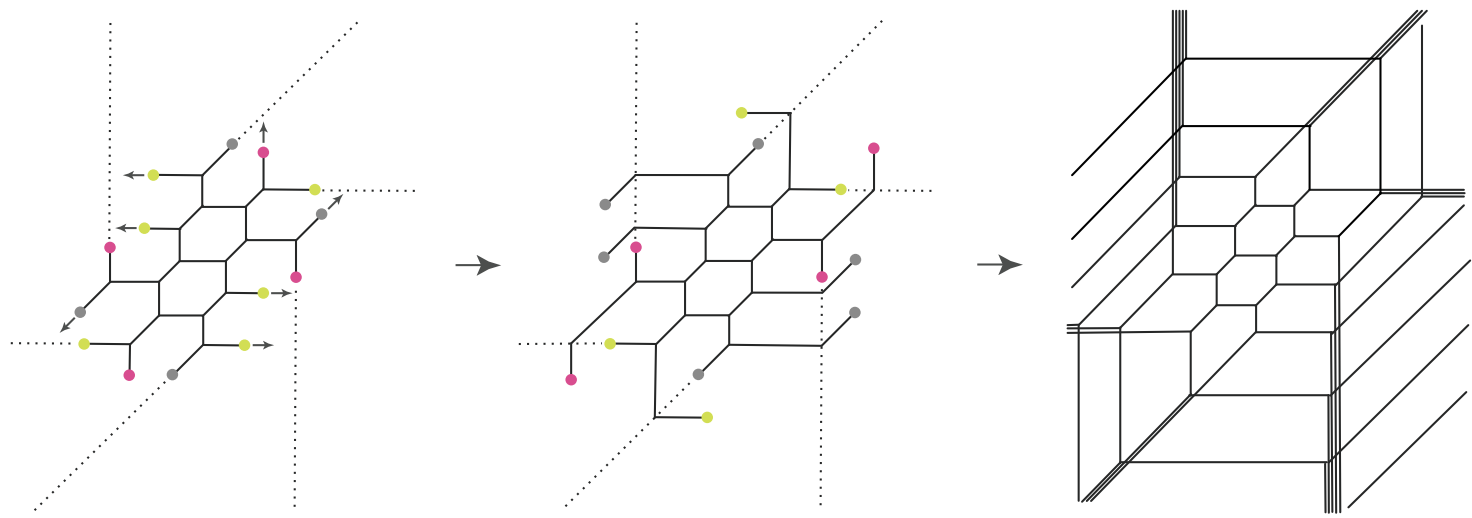

Figure 3. Tao diagram for $5 \mathrm{~d} \mathcal{N}=1 \mathrm{SU}(3)$ gauge theory with $N_{f}=10$ flavors which is a circle compactification of $6 \mathrm{~d} \mathcal{N}=(1,0) \operatorname{Sp}(1)$ gauge theory with one tensor multiplet and 10 flavors. The rightmost brane configuration on figure 2 can be reorganized to be the leftmost web configuration given here, using 7-brane monodromies. Pulling out 7-branes passing through the branch cut of other 7-branes gives a Tao wed diagram on the rightmost.

fundamental domain of the 5-brane web diagram that is confined by "the mirror reflection lines" is expanded to the two-dimensional plane and the boundary made by NS5 branes with the mirror reflection lines forms " 5 -brane loops" [37]. (See figure 2.)

Given such a $(p, q)$ 5-brane configuration with 7-branes and 5-brane loops, one has a 5 -dimensional gauge theory description by pulling all the 7-branes to infinity, while keeping the gauge coupling finite as pulling out all the 7-branes. 5-branes are naturally generated as a result of the Hanany-Witten effect such that a 7-brane is attached to the end of an external 5-brane. Hence, $2 N+8$ D7-branes give rise to semi-infinite $2 N+8$ D5-branes yielding the flavor branes of the theory. Due to the charge conservation at a junction of the web diagram, 5-brane loops induce two more color D5-branes. This means that the resulting $5 \mathrm{~d}$ gauge theory in this process has $N+2$ color branes, implying that the rank of the resulting $5 \mathrm{~d}$ gauge theory is increased by one. For this, one can also argue that the $6 \mathrm{~d}$ tensor multiplet contributes the $5 \mathrm{~d}$ vector multiplet giving one more rank to the $5 \mathrm{~d}$ gauge group. It is worth noting that the obtained $5 \mathrm{~d}$ theory has zero CS level. As the CS level can be measured as the angle difference of two vertically inclined 5-branes playing a role of NS5-brane in the web configuration, the angle between the two upper external 5-branes and the angle between the two lower external 5-branes are the same as the one between as shown in figure 2, confirming the zero CS level of the $5 \mathrm{~d}$ theory. Therefore, the resultant 5 d theory is $\mathcal{N}=1 \mathrm{SU}(N+2)_{0}$ gauge theory with $N_{f}=2 N+8$ flavors.

The web diagram for this case was already studied by the authors in [27] and is named as a Tao web diagram. Such Tao diagram obtained by pulling all the 7-branes outside the 5-brane loops has the necessary structures to be interpreted as a circle compactification of the $6 \mathrm{~d}$ theory. It is of the shape of an infinitely repeated spiral web with a constant period, as given in figure 3 . This period can be expressed in terms of the parameter of the $5 \mathrm{~d}$ theory, the instanton factor, which is an expected property for a circle compactification of $6 \mathrm{~d}$ theory. The spectrum coming from infinitely expanding 5-branes are naturally identified as the KK spectrum of the compactification. For a short introduction, see appendix A or [27, 30]. 


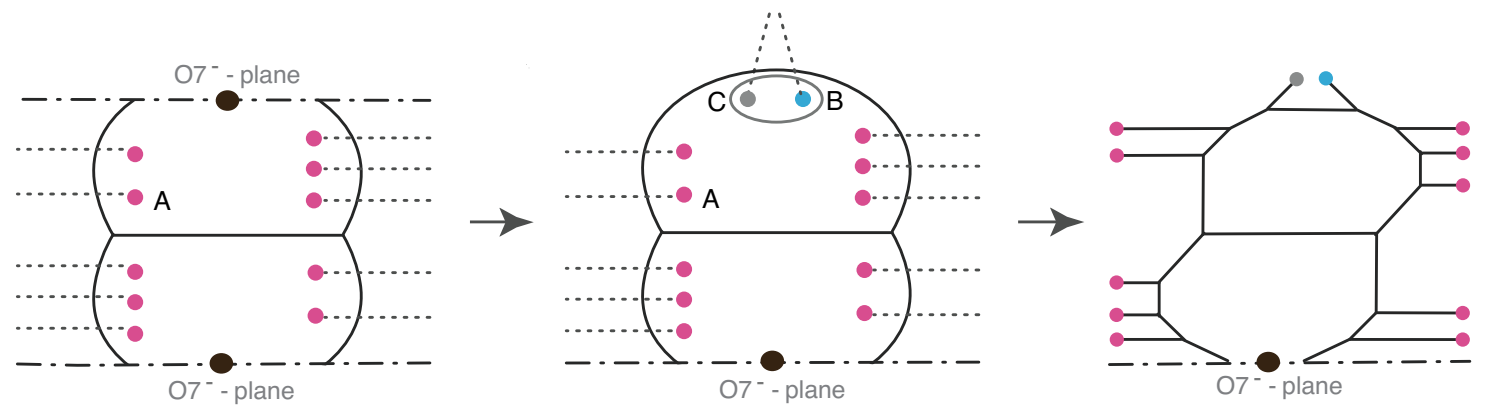

Figure 4. Type IIB brane descriptions for $6 \mathrm{~d} \mathcal{N}=(1,0) \operatorname{Sp}(N)$ gauge theory with one tensor multiplet and $2 N+8$ flavors in the fundamental representation, which yields $\mathcal{N}=1 \operatorname{Sp}(N+1)$ gauge theory with the number of flavors. For simplicity, $N=1$. Left: the brane configuration with two $\mathrm{O7}^{-}$planes. Middle: the brane configuration with only one of two $\mathrm{O7}^{-}$planes is resolved. Right: the resulting $\operatorname{Sp}(2)$ gauge theory with $N_{f}=10$ flavors.

\section{$2.25 \mathrm{~d} \operatorname{Sp}(N+1)$ theory with $N_{f}=2 N+8$ flavors}

While the quantum resolution of both $O 7^{-}$planes leads to the $5 \mathrm{~d} \mathrm{SU}(N+2)$ gauge theory with $2 N+8$ flavors, one may resolve only one of the two $O 7^{-}$planes which arise from T-duality of the $08^{-}$plane in Type IIA brane picture of $6 \mathrm{~d} \operatorname{Sp}(N)$ theory. In this process of reducing the $6 \mathrm{~d}$ theory to $5 \mathrm{~d}$, the Wilson line is introduced such that all the D5-branes are located above the unresolved $O 7^{-}$plane, to retain an $S p$ gauge group. As discussed earlier, a resolution of the $\mathrm{O}^{-}$plane into a pair of two 7-branes induces an extra color D5 brane when 7 -branes are pulled out to infinity. Hence, the resulting $5 \mathrm{~d}$ theory is $\mathcal{N}=1$ $\operatorname{Sp}(N+1)$ gauge theory with the same number of flavors, $N_{f}=2 N+8$. The increase of the rank of the gauge group can be again due to the contribution of one $6 \mathrm{~d}$ tensor multiplet. A brane setup for the $5 \mathrm{~d} \operatorname{Sp}(N+1)$ gauge theory in the Coulomb branch is given in figure 4 .

As $\pi_{4}(\operatorname{Sp}(n))=\mathbb{Z}_{2}$, there is a discrete theta parameter for $\operatorname{Sp}(n)$ gauge theory. This parameter can be interpreted as two inequivalent resolutions of the $O 7^{-}$plane if the $\operatorname{Sp}(n)$ gauge theory has no flavors. When the $\operatorname{Sp}(n)$ gauge theory has flavors, the effect of the discrete theta parameter can be absorbed by a redefinition of mass parameters and the difference is not physical [38]. Therefore, one can choose whatever splitting of the $O 7^{-}$plane. For example, one can resolve the $O 7^{-}$plane with a pair of $[1,1]$ and $[1,-1] 7$-branes as chosen earlier, and one can also resolve it with a pair of $[2,1]$ and $[0,1] 7$-branes.

\subsection{Equivalence between $\mathrm{Sp}$ an $\mathrm{SU}$ theories and their flavor decoupling limits}

In the preceding subsections, we have discussed a circle compactification of $6 \mathrm{~d} \mathcal{N}=(1,0)$ $\operatorname{Sp}(N)$ gauge theory with $N_{f}=2 N+8$ flavor and one tensor multiplet in two ways with respect to the resolution of the $O 7^{-}$-planes as well as the Wilson lines: one of which leads to the $5 \mathrm{~d} \mathrm{SU}(N+2)$ theory involving the resolution of two $O 7^{-}$-planes, and the other leads to the $5 \mathrm{~d} \operatorname{Sp}(N+1)$ theory involving the resolution of only one $O 7^{-}$-plane. Both have the same number of flavors as the 6d theory, as the flavors are associated with D7-branes which come from a T-dual of D8-branes in the brane picture of the $6 \mathrm{~d}$ theory. Note that 

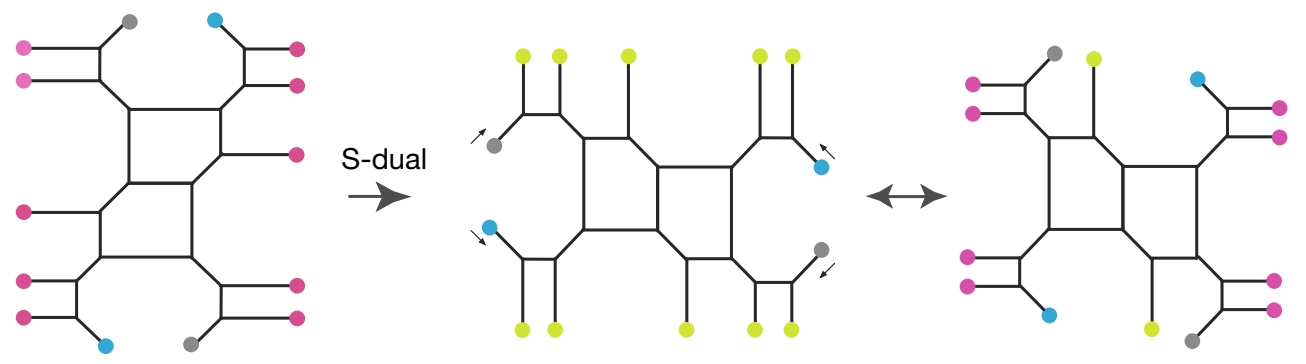

Figure 5. The S-dual of $\mathrm{SU}(3)$ theory with $N_{f}=10$ flavor is $\mathrm{SU}(2) \times \mathrm{SU}(2)$ quiver theory with $N_{f}=4$ flavors to each $\mathrm{SU}(2)$ theory of the quiver.

global symmetry for both theories is $\mathrm{SO}(4 N+16) \times \mathrm{U}(1)_{I}$ where $\mathrm{SO}(4 N+16)$ is the global symmetry of $6 \mathrm{~d}$ theory and $\mathrm{U}(1)_{I}$ comes from the circle compactification.

As both have the same UV completion as the $6 \mathrm{~d}$ SCFT, $\left(D_{N+4}, D_{N+4}\right)$ minimal conformal matter theory, and thus the same global symmetry, we claim that these two theories are dual to each other at the UV. Hence, some physical observables like index functions would be equivalent. For instance, it would be interesting to see the partition functions, or elliptic genera, of both theories do agree or not.

Interestingly, the superconformal index for the $5 \mathrm{~d} \mathrm{SU}(N+2)$ theory and the $\mathrm{Sp}(N+1)$ theory with less flavors $N_{f} \leq 2 N+7$ which have the $5 \mathrm{~d}$ UV fixed point, was computed [34], showing the equivalence of the two indices. In the web diagram, this equivalence with the flavors $N_{f} \leq 2 N+7$ can be easily explained if one considers the flavor decoupling of the $5 \mathrm{~d}$ theories from the critical flavors. Namely, one can take the mass decoupling limit by deforming the mass of a flavor to an extreme value of either $\infty$ or $-\infty$.

We note that in order to hold the $5 \mathrm{~d}$ dualities for the less flavor cases, the CS level of $\mathrm{SU}(N+2)$ theory should be either maximum or minimum. This comes from the fact that we need to decouple the flavors exactly in the same way between the $5 \mathrm{~d} \mathrm{SU}(N+2)$ gauge theory and the $5 \mathrm{~d} \operatorname{Sp}(N+1)$ gauge theory. For example, from the figure 4, we can take the mass of the flavors only to $+\infty$ for the $\operatorname{Sp}(N+2)$ gauge theory. Therefore, we should also take the same flavor decoupling limit for the $5 \mathrm{~d} \mathrm{SU}(N+2)$ gauge theory.

\subsection{S-dualities}

In the $(p, q) 5$-brane web diagram, the S-duality structure of the theory is manifest as one rotates the $(p, q) 5$-brane web by 90 degrees, namely, D5-branes become NS5-branes and NS5-branes become D5-branes. A simple example would be the S-duality of the SU(3) theory which yields the quiver theory of $\mathrm{SU}(2) \times \mathrm{SU}(2)$. For instance, the brane configuration on the right of figure 2 describes a $5 \mathrm{~d} \mathrm{SU}(3)$ theory with $N_{f}=10$ flavors, and then its S-dual theory is the [4] $-\mathrm{SU}(2) \times \mathrm{SU}(2)-[4]$ quiver theory which has four flavors ${ }^{5}$ (denoted in the square bracket "[ ]") on each gauge group as in figure 5. In such Tao

\footnotetext{
${ }^{5}$ The middle configuration of figure 5 has two "SU(1)"s linked to each $\mathrm{SU}(2)$ instead of four flavors. Such SU(1) has the instanton factor which can be used to express the period of the Tao web diagram. On the other hand, using 7-brane monodromy analysis, it can be shown that such SU(1) factor together with a bi-fundamental hypermultiplet connecting between the $\mathrm{SU}(1)$ and $\mathrm{SU}(2)$ also represents two flavors.
} 


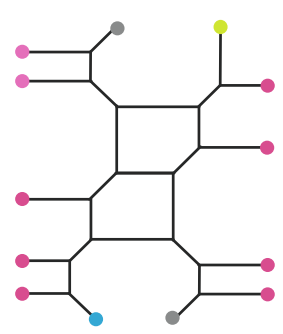

(a)

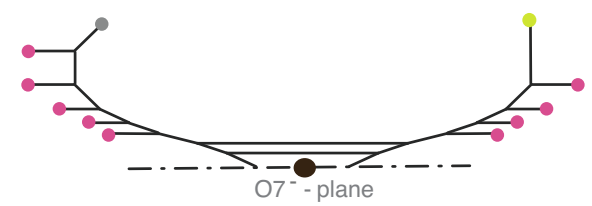

(b)

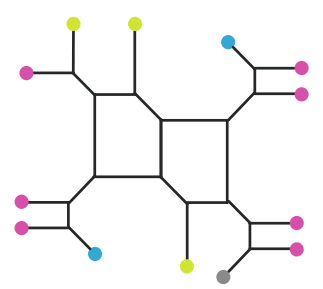

(c)

Figure 6. Mass decoupling limit. (a) $\mathrm{SU}(3)_{\frac{1}{2}}$ theory with $N_{f}=9$ flavors and the CS level $\frac{1}{2}$; (b) $\mathrm{Sp}(2)$ theory with $N_{f}=9$ flavors and $k=0 ;$ (c) [3] $-\mathrm{SU}(2)-\mathrm{SU}(2)-[4]$ quiver theory.

diagrams of figure 2, as discussed, the constant period of spiral shape is expressed in terms of the instanton factor of the gauge theory. It holds for the S-dual picture, the constant period is expressed in terms of instantons factors of the quiver theory [27]. This S-duality generalizes to higher rank cases so that the $\mathrm{S}$-dual of the $5 \mathrm{~d} \mathrm{SU}(N+2)$ gauge theory with $N_{f}=2 N+8$ flavors is given by the $\mathrm{SU}(2)$ quiver theory

$$
[4]-\underbrace{\mathrm{SU}(2)-\cdots-\mathrm{SU}(2)}_{N+1 \text { nodes }}-[4] .
$$

The period for these Tao diagram is expressed in terms of a product of the instanton factors of each gauge node.

We summarize the $5 \mathrm{~d}$ theories obtained by compactifying the $6 \mathrm{~d} \operatorname{Sp}(N)$ gauge theory with $N_{f}=2 N+8$ and one tensor multiplet, on a circle:

(i) $\mathrm{SU}(N+2)-[2 N+8], \mathrm{SU}(N+2)$ gauge theory with $N_{f}=2 N+8$ flavors,

(ii) $\operatorname{Sp}(N+1)-[2 N+8], \operatorname{Sp}(N+1)$ gauge theory with $N_{f}=2 N+8$ flavors,

(iii) $[4]-\mathrm{SU}(2)-\cdots-\mathrm{SU}(2)-[4]$, a quiver theory with $N+1 \mathrm{SU}(2)$ gauge nodes and 4 flavors are attached to each boundary node.

The (i) and (ii) are obtained by resolving either both two $O 7^{-}$planes or only one $O 7^{-}$ plane when reducing to $5 \mathrm{~d}$, and (iii) is S-dual of (i). Therefore, (iii) is also (S-)dual to (ii).

Decoupling flavors. As discussed in [27], one can decouple flavors of a given Tao diagram, which yields 5d supersymmetric gauge theory which has the $5 \mathrm{~d}$ UV fixed point. In $\operatorname{Sp}(N+1)$ or $\mathrm{SU}(N+2)$ gauge theories, we can decouple the same number of flavors. The same idea applies to the $\mathrm{SU}(2) \times \mathrm{SU}(2)$ quiver theory. The resulting flavor decoupled theories from $\mathrm{SU}(N+1), \mathrm{Sp}(N+1)$, and the $\mathrm{SU}(2) \times \mathrm{SU}(2)$ quiver theories are all dual to each other, and are expected to have the same $5 \mathrm{~d}$ UV fixed point.

\section{$36 \mathrm{SU}(N)$ gauge theory with $N_{f}=N+8$ and $N_{a}=1$}

In this section, we consider six-dimensional $\mathrm{SU}(N)$ gauge theory with $N_{f}=N+8$ flavors and $N_{a}=1$ hypermultiplet in antisymmetric representation together with one tensor multiplet. This theory has $\mathrm{SU}(N+8) \times \mathrm{U}(1)$ anomaly free global symmetry. 

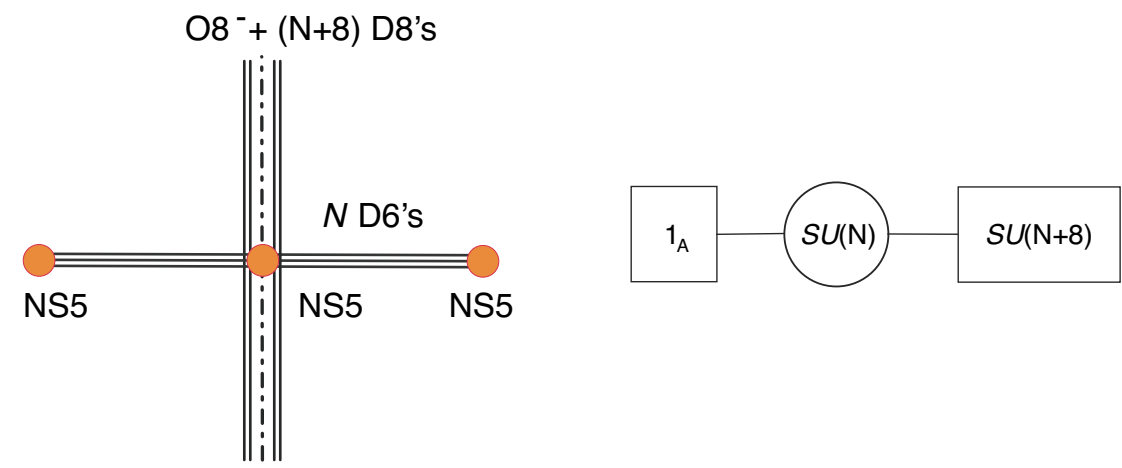

Figure 7. Left: type IIA brane set up for $6 \mathrm{~d} \mathrm{SU}(N)$ gauge theory with $N_{f}=N+8$ and $N_{a}=1$. Right: corresponding quiver diagram.

When $N$ is small, there can be the enhancement. When $N=3$, the anti-symmetric representation is equivalent to the (anti-)fundamental representation. Therefore, this theory is interpreted as $\mathrm{SU}(3)$ gauge theory with 12 flavors. In this case, the global symmetry $\mathrm{SU}(11) \times \mathrm{U}(1)$ actually enhances to $\mathrm{SU}(12)$. When $N=4$, anti-symmetric representation is the real representation. Therefore, we can consider the $\mathrm{SU}(2)$ global symmetry acting on two half hypermultiplets. In this case, the global symmetry $\mathrm{SU}(12) \times \mathrm{U}(1)$ actually enhances to $\mathrm{SU}(12) \times \mathrm{SU}(2)$. The discussion below is similar to that in section 2. Depending on resolving both or only one $O 7^{-}$-plane(s), we obtain two different $5 \mathrm{~d}$ descriptions. We also discuss various $5 \mathrm{~d}$ dual descriptions corresponding to this $6 \mathrm{~d}$ theory.

\section{$3.15 \mathrm{~d}$ SU $\times$ SU gauge theory with flavors}

$6 \mathrm{~d} \mathrm{SU}(N)$ gauge theory with $N_{f}=N+8, N_{a}=1$ and with one tensor multiplet is realized by the brane set up depicted in figure 7 . Compared to figure 1, there is an additional NS5-brane on top of the $\mathrm{O}^{-}$plane. Moreover, odd number of D6-branes are also allowed in this case.

We compactify one of the directions which NS5-brane extends and take T-duality along this direction. Then, we obtain the IIB brane setup depicted in figure 8 , where we chose the fundamental region to be the half of the compactified $S^{1}$ direction. Compared to 2, we have additional $(p, q)$ 5-brane connecting two $O 7^{-}$planes, where $n$ D5-branes are placed on the left hand side and $(N-n)$ D5-branes are place on the right hand side of this 5 -brane. We note that this "distribution" labeled by $n$ depends on the Wilson line of the $\mathrm{SU}(N)$ gauge group which we introduce when we compactify on $S^{1}$. Accordingly, the charge of the 5-branes attached to the $O 7^{-}$planes depends on this distribution. Likewise, the D7branes which are T-dual of the D8-branes are also distributed in analogous way into $n^{\prime}$ and $N+8-n^{\prime}$.

Here, we would like to consider the quantum resolution for one of the $O 7^{-}$planes based on [35]. The decomposition in this case is, however, more non-trivial than that on discussed in section 2, because the $O 7^{-}$planes here are attached to the 5-branes. As discussed in [39], one needs to properly choose the charge of the resolved two 7-branes in such a way that one of the 7 -branes should be attached to the 5 -brane that is attached to the $\mathrm{O}^{-}$-plane 


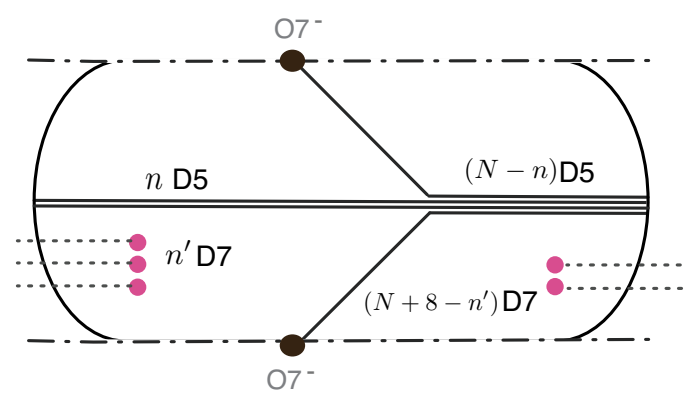

Figure 8. A T-dual description of figure 7.

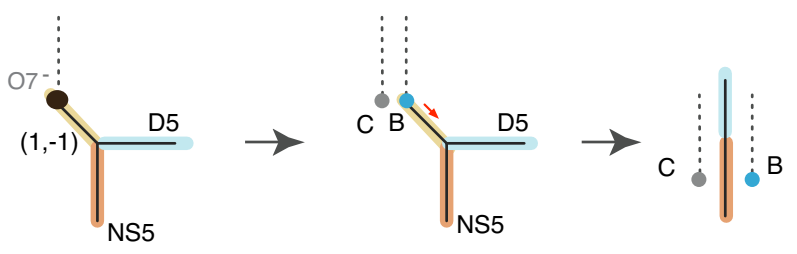

Figure 9. Resolution of the $O 7^{-}$plane attached to $(1,-1)$ 5-brane. A $[1,-1]$ 7-brane is denoted by $\mathbf{B}$ and a $[1,1]$ 7-brane is denoted by $\mathbf{C}$.

before the resolution. For example, when an $O 7^{-}$plane is attached to $(1,-1) 5$-brane as depicted on the left of figure 9 , the $O 7^{-}$plane can be decomposed into $\mathbf{B}=[1,-1] 7$-brane and $\mathbf{C}=[1,1] 7$-brane so that $\mathbf{B} 7$-brane is attached to the $(1,-1) 5$-brane. ${ }^{6}$ Once the $O 7^{-}$-plane is resolved, 7 -branes induce new configurations. Imagine, for example, a 5-brane junction composed of an NS5-brane, a D5-brane and a $(1,-1)$ 5-brane which is connected to $\mathbf{B}$ 7-brane as in the middle of figure 9. By moving $\mathbf{B}$ 7-brane along the direction of the $(1,-1)$ 5-brane using Hanany-Witten transition, one can transform the configuration into a brane configuration where the $\mathbf{B}$ 7-brane becomes fee so that it is not attached to the 5-brane. As a result, $\mathbf{B}$ and $\mathbf{C}$ 7-branes are separated by the NS5-brane as depicted on the right of figure 9. This kind of transforming procedure is useful in obtaining various five-dimensional (5d) field theory descriptions after resolving $O 7^{-}$-planes.

As discussed in section 2, the quantum resolution of $\mathrm{O}^{-}$-planes generates new 5-branes to make a closed 5-brane loop. The resulting brane configuration is depicted on the left side of figure 10, where two $O 7^{-}$-planes are resolved into two 7-branes and one of which is attached to a 5-brane is moved along the direction of the charge of the 5-brane to be a free 7-brane as described. To obtain a $5 \mathrm{~d}$ gauge theory description for this brane configuration, one can move around one of the D7-branes passing through D5-branes as well as 7-branes toward outside the 5-brane loop. (see the arrows attached to D7-branes in figure 10.) Then it leads to a 5 -brane web diagram depicted on the right side of figure 10 . We note that a $5 \mathrm{~d}$ field theory description is possible only when the newly generated 5-branes are D5-brane,

\footnotetext{
${ }^{6} \mathrm{It}$ is also possible for one to resolve the $O 7^{-}$-plane into $[3,-1]$ and $[1,-1]$ 7-branes as the total monodromy is the same as that of an $O 7^{-}$-plane. In this case, however, one can rearrange the 7-branes so as to yield the same configuration with $\mathbf{B}$ and $\mathbf{C}$ 7-branes.
} 

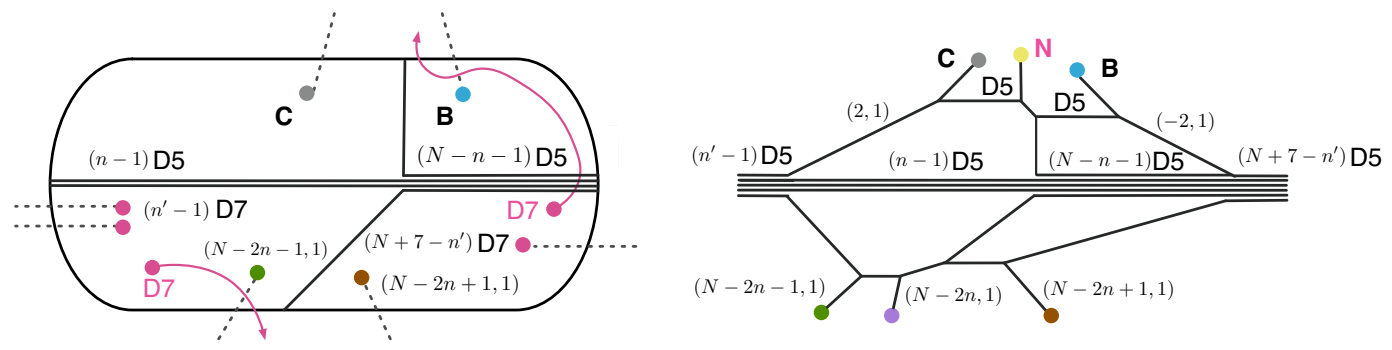

Figure 10. Left: the two $O 7^{-}$planes in figure 8 is resolved. Right: web-diagram obtained by moving all the 7-branes outside.

provided that the two distribution parameters, the number of the color D5-branes $n$ and the number of the flavor D7-branes $n^{\prime}$, are constrained by $n^{\prime}=3 n+4-N$. With a suitable Wilson line for the $\mathrm{SU}\left(N_{f}\right)$ flavor group, the brane configuration on the right of figure 10 gives rise to the $5 \mathrm{~d}$ quiver gauge theory

$$
[3 n+3-N]-\mathrm{SU}(n+1)_{0}-\mathrm{SU}(N+1-n)_{0}-[2 N+3-3 n]
$$

where as the distribution parameter, the integer $n$ should be chosen in such a way that all the flavors and ranks of the gauge groups of this theory should be positive. With such $n$, we claim that any resulting $5 \mathrm{~d}$ quiver gauge theory is a possible $5 \mathrm{~d}$ description for $6 \mathrm{~d}$ $\mathrm{SU}(N)$ gauge theory with $N_{f}=N+8, N_{a}=1$ and with one tensor multiplet compactified on $S^{1}$. In other words, the theories with different $n$ should have the identical $6 \mathrm{~d}$ UV fixed point. We denote this equivalence as "distribution duality." We will discuss this distribution duality more in detail in section 3.5.

\section{$3.25 \mathrm{~d} \mathrm{SU}(N+1)$ gauge theory with $N_{f}=N+7$ and $N_{a}=1$}

Analogous to section 2.2, we can decompose only one $O 7^{-}$-plane out of the two $O 7^{-}$-planes in figure 8. Suppose we resolve the upper $\mathrm{O}^{-}$-plane from 8 , then we get the configuration on the left of figure 11. As discussed in the previous subsection, we can take a D7-brane through to locate between $\mathbf{B}$ and $\mathbf{C}$ 7-branes, which convert the $[p, q]$ charge of the D7brane to be $\mathbf{N}=[0,1]$. This leads to the configuration on the right of figure 11 . This configuration corresponds to adding the flavor branes to the one discussed in [39].

The resulting $5 \mathrm{~d}$ description is a $5 \mathrm{~d} \mathrm{SU}(N+1)_{0}$ gauge theory with $N_{f}=N+7$ flavor and $N_{a}=1$ antisymmetric hypermultiplet coming from the 5-brane connected to unresolved $\mathrm{O}^{-}$-plane. Notice that the number of fundamental hypermultiplet is reduced by one, since we used up a D7-brane to convert to a $[0,1] 7$-brane which is essential to have a brane configuration with an antisymmetric hypermultiplet. Therefore, we conclude that $6 \mathrm{~d} \mathrm{SU}(N)$ gauge theory with $N_{f}=N+8, N_{a}=1$ and with one tensor multiplet compactified on $S^{1}$ is also described by $5 \mathrm{~d} \mathrm{SU}(N+1)_{0}$ gauge theory with $N_{f}=N+7$ flavors and $N_{a}=1$. 

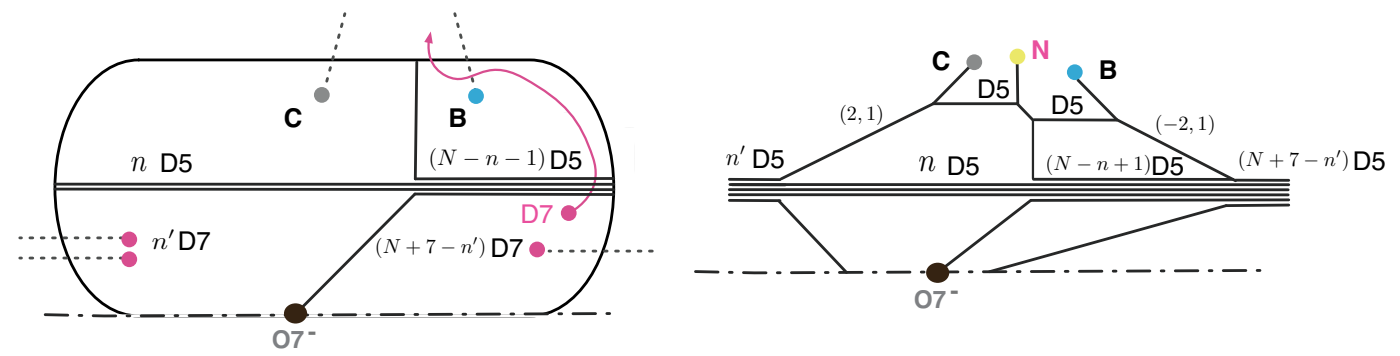

Figure 11. $5 \mathrm{~d} \mathrm{SU}(N+1)_{0}$ gauge theory with $N_{f}=N+7$ and $N_{a}=1$

\subsection{Equivalence of the two $5 \mathrm{~d}$ descriptions and their flavor decoupling limits}

In the previous subsections, we discussed two different $5 \mathrm{~d}$ descriptions for the $6 \mathrm{~d} \mathrm{SU}(N)$ gauge theory with $N_{f}=N+8, N_{a}=1$ and one tensor multiplet, which are summarized as

(i) $5 \mathrm{~d}[3 n+3-N]-\mathrm{SU}(n+1)_{0}-\mathrm{SU}(N+1-n)_{0}-[2 N+3-3 n]$;

(ii) $5 \mathrm{~d} \mathrm{SU}(N+1)_{0}$ gauge theory with $N_{f}=N+7$ and $N_{a}=1$.

These two descriptions are dual in the sense that they have the same $6 \mathrm{~d}$ UV fixed point. Furthermore, by considering the flavor decoupling limit by taking some of the mass parameters to be infinity, we will again obtain the dual theories, which then have again the identical $5 \mathrm{~d}$ fixed point.

For instance, in figure 10, let us take $n_{L}$ flavors out of the left flavors of the quiver theory (i), $[3 n+3-N]$, and $n_{R}$ flavors out of the right flavors of (i), $[2 N+3-3 n]$. By giving heavy masses to $n_{L}$ and $n_{R}$ flavors, corresponding to moving these $n_{L}$ and $n_{R}$ flavor branes upward in figure 10, we can decouple $n_{L}$ and $n_{R}$ flavors from the brane configuration, which reduces the left and right flavors to be $3 n+3-N-n_{L}$ and $2 N+3-3 n-n_{R}$, respectively. This also alter the CS levels of $\mathrm{SU}(n+1) \times \mathrm{SU}(N+1-n)$ to be shifted by $n_{L}$ and $n_{R}$ respectively. After the flavor decoupling, we get

$$
5 \mathrm{~d}\left[3 n+3-N-n_{L}\right]-\mathrm{SU}(n+1)_{n_{L}}-\mathrm{SU}(N+1-n)_{n_{R}}-\left[2 N+3-3 n-n_{R}\right] .
$$

In a similar fashion, we can decouple some flavors of (ii). Say, we give the positive infinite masses to $n_{L}$ flavors from the left $n^{\prime}=3 n+4-N$ flavors and $n_{R}$ flavors from the right $N+7-n^{\prime}$ flavors of the brane configuration on the right of figure 11. This also shifts the CS level of $\mathrm{SU}(N+1)$ to be $n_{L}-n_{R}$ and hence reduces the theory to be

$$
5 \mathrm{~d} \quad \mathrm{SU}(N+1)_{n_{L}-n_{R}} \text { with } N_{f}=N+7-\left(n_{L}+n_{R}\right) \text { and } N_{a}=1 .
$$

As the way we decouple the flavors from (i) and (ii) are same, we expect that two $5 \mathrm{~d}$ theories (3.2) and (3.3) obtained from the flavor decoupling should have the identical $5 \mathrm{~d}$ UV fixed point, giving rise to another non-trivial 5 d duality.

\subsection{S-dualities and mass parameters}

By using S-duality, we can obtain more 5d descriptions which have the identical UV fixed point. In this subsection, we consider S-dual description for the quiver theory discussed in 

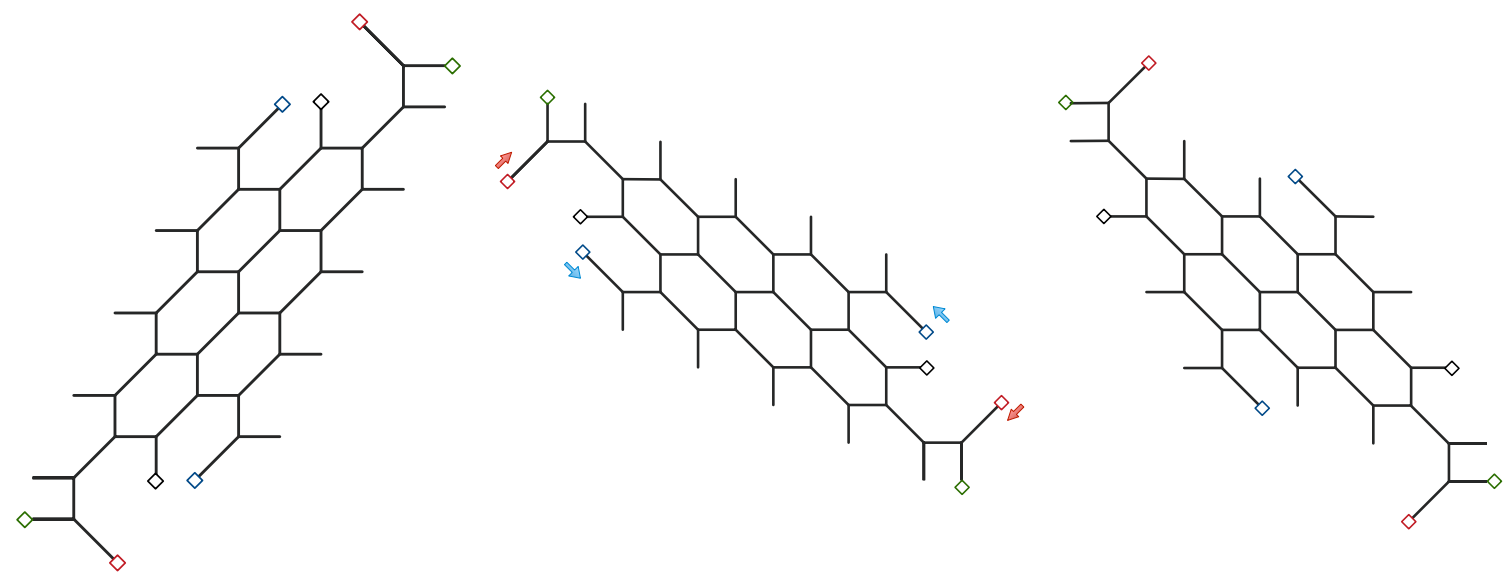

Figure 12. S-transformation of the 5 d quiver gauge theory $[6]-\mathrm{SU}(4)_{0}-\mathrm{SU}(4)_{0}-[6]$.

section 3.1. We discuss the dual descriptions with a specific example, and generalization is then straightforward. Take $N=6$ and $m=3$, then the 5 d quiver gauge theory (3.1) becomes

$$
[6]-\mathrm{SU}(4)_{0}-\mathrm{SU}(4)_{0}-[6]
$$

The corresponding web diagram is depicted on the left diagram of figure 12 .

Parallel to section 2.4, we first consider the S-duality transformation exchanging D5branes and NS5-branes to each other, which corresponds to a 90-degree rotation of the diagram. We denote this type of S-duality as "S-transformation". Acting the S-transformation to the left diagram on figure 12 , we obtain the middle diagram of figure 12 . In this middle diagram, we move $[1,1]$-branes and $[1,-1]$ 7-branes along with the Hanany-Witten transition, then we obtain the following $5 \mathrm{~d}$ quiver gauge theory

$$
[5]-\mathrm{SU}(3)_{0}-\mathrm{SU}(3)_{0}-\mathrm{SU}(3)_{0}-[5]
$$

where the corresponding web diagram depicted on the right of figure 12 .

Here, we can consider another type of S-duality, which transforms D5 branes, NS5 branes, and $(1,1) 5$-branes into $(1,-1) 5$-branes, NS5 branes, and D5 branes, respectively. We denote this as "STS transformation", which roughly corresponds to a 45-degree rotation of the diagram. We start from the same theory (3.4), but for convenience we consider the web diagram on the left of figure 13, which is related to the left diagram of figure 12 by a simple Hanany-Witten transition to move the right top and left bottom D7-branes to the NS5 brane inward. After taking the STS transformation and then moving [0,1] 7-branes upward or downward, we obtain the 5 d quiver gauge theory

$$
\begin{array}{cc}
{[1]} & {[1]} \\
{[3]-\mathrm{SU}(2)-\mathrm{SU}(3)_{0}-\mathrm{SU}(3)_{0}-\mathrm{SU}(2)-[3],}
\end{array}
$$

which is different from the usual S-daulized theory (3.5). In this way, we can obtain several $5 \mathrm{~d}$ descriptions by different types of S-duality transformation. 

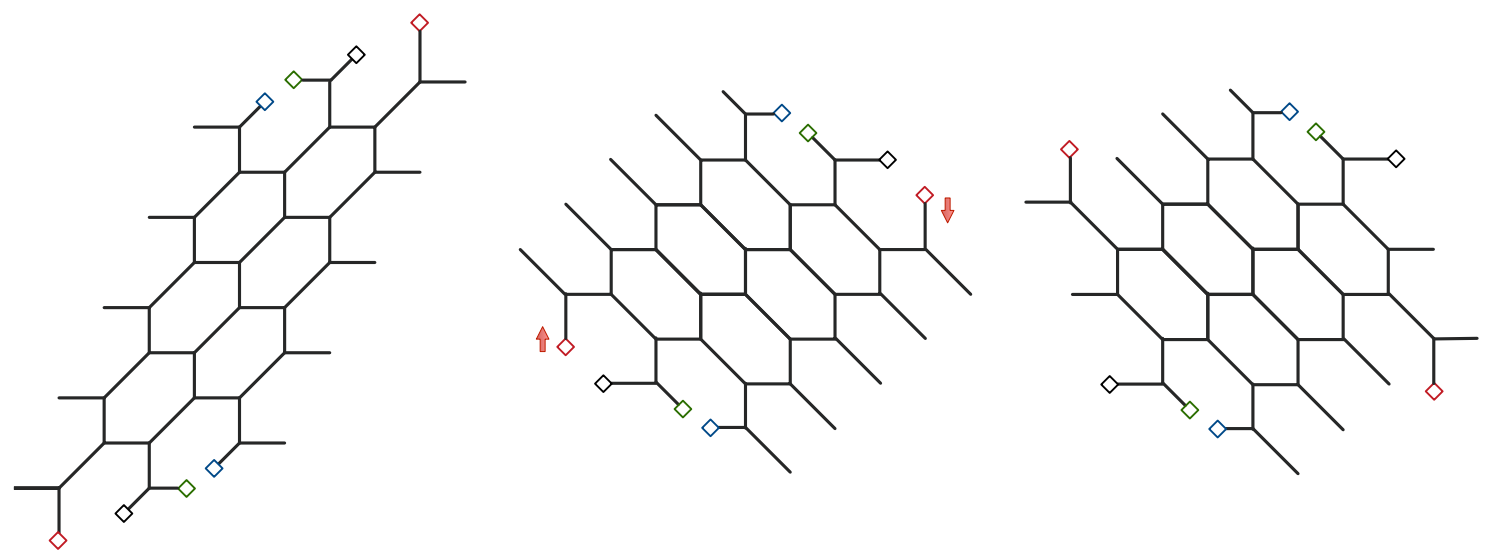

Figure 13. STS-transformation of the 5 d quiver gauge theory $[6]-\mathrm{SU}(4)_{0}-\mathrm{SU}(4)_{0}-[6]$.

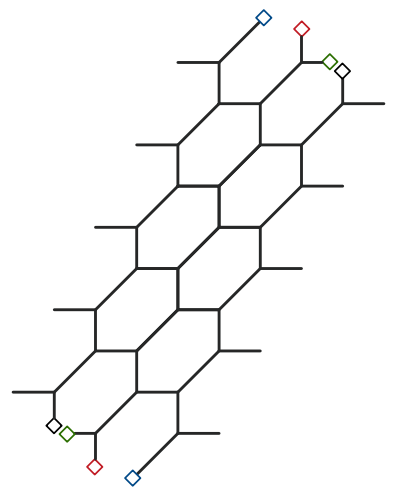

Figure 14. Same theory with different mass parameter

We remark here that a given gauge theory may have web diagrams withe different ranges of mass parameters. For example, instead of starting from the left of figure 12 or figure 13, we could have started from the diagram on figure 14, which describe the same theory but with different mass parameters. The existence of different web configuration leads to an interesting yet another dual description. Namely, although the original $5 \mathrm{~d}$ description is identical up to the value of the mass parameters, one may obtain different results under the S-duality transformation. For instance, it is straightforward to see that the S-transformation yields 5d quiver gauge theory (3.6) rather than (3.5). Analogously, the STS-transformation gives the theory (3.5) rather than 3.6. It is worth emphasizing that depending on the value of the mass parameters of the flavors, the same $5 \mathrm{~d}$ gauge theory can be mapped to different gauge theories under the identical S-duality transformation.

\subsection{Distribution duality}

In section 3.1, we discussed that $5 \mathrm{~d}$ quiver gauge theories (3.1) with different values of $n$ have an identical $6 \mathrm{~d}$ UV fixed point, referred to the distribution duality. We devote ourselves in this subsection to discuss the distribution duality in more details from the viewpoint of type IIB 5-brane web diagram. 


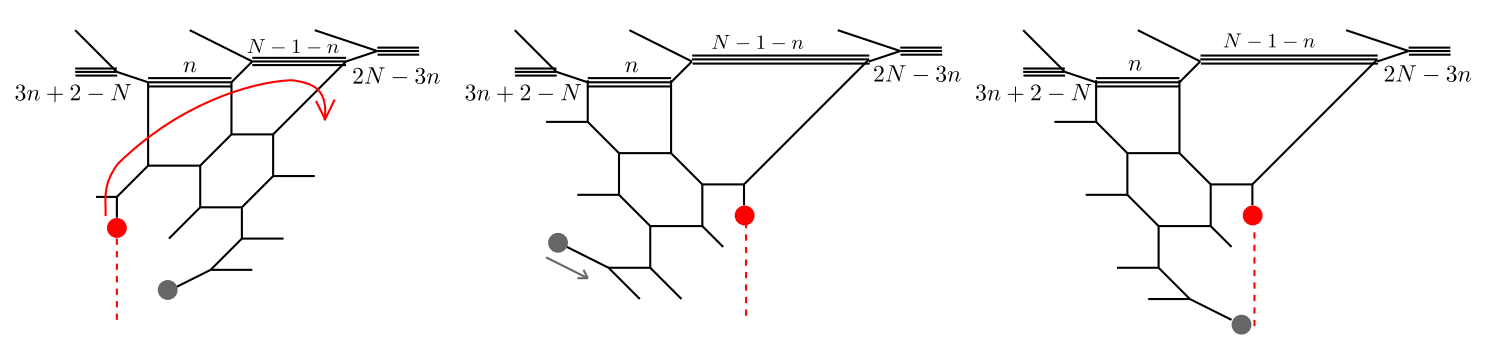

Figure 15. Derivation of the distribution duality.

We start from the left diagram in figure 15, which describes the $5 \mathrm{~d}$ quiver gauge theory (3.1). Then, we move the $[0,1] 7$-brane painted in red along the arrow in the leftmost diagram in figure 15. In this process, the charges of 5-branes in the lower part of the diagram are modified because they went across the monodromy cut generated by the $[0,1] 7$-brane. This changes the structure of the web diagram so that the resulting diagram gives rise to the following $5 \mathrm{~d}$ quiver gauge theory

$$
[3 n+6-N]-\mathrm{SU}(n+2)_{0}-\mathrm{SU}(N-n)_{0}-[2 N-3 n] .
$$

We find that this theory is the one obtained by shifting $n$ by 1 in the quiver gauge theory (3.1). The repetition of this procedure yields that the quiver gauge theories (3.1) and (3.7) with an arbitrary $n$ are dual to each other as long as all the numbers of flavor and the rank of the gauge groups are positive.

By considering the flavor decoupling limit discussed in section 3.3, it is also straightforward to show that this distribution duality holds also for the theories with 5 dimensional UV fixed points. Since the process depicted in figure 15 does not depend on the upper part of the diagram, the discussion is parallel even after we decouple $n_{1}$ and $n_{2}$ flavors coupling to the two gauge groups. We see that the following two theories are again related by the analogous procedure

- $5 \mathrm{~d}\left[3 n+3-N-n_{1}\right]-\mathrm{SU}(n+1)_{n_{1}}-\mathrm{SU}(N+1-n)_{n_{2}}-\left[2 N-3 n+3-n_{2}\right]$

- $5 \mathrm{~d}\left[3 n+6-N-n_{1}\right]-\mathrm{SU}(n+2)_{n_{1}}-\mathrm{SU}(N-n)_{n_{2}}-\left[2 N-3 n-n_{2}\right]$

We can further consider moving the flavor branes upward. Thus, this class of theories with all the possible value $n$ are related by the distribution duality.

Up to here, we have discussed the flavor decoupling limit, which corresponds to moving the flavor branes upward in figure 15. We can also consider another type of flavor decoupling limit corresponding to moving flavor branes downward.

As an example, suppose that we move one of the flavor branes coupled to $\mathrm{SU}(N+1-n)$ downward in the left of figure 15 to downward. Then, we obtain the left diagram in figure 16. By considering the analogous procedure, we find the following two are related

- $5 \mathrm{~d}[3 n+3-N]-\mathrm{SU}(n+1)_{0}-\mathrm{SU}(N+1-n)_{-\frac{1}{2}}-[2 N-3 n+2]$

- $5 \mathrm{~d}[3 n+5-N]-\mathrm{SU}(n+2)_{-\frac{1}{2}}-\mathrm{SU}(N-n)_{0}-[2 N-3 n]$ 

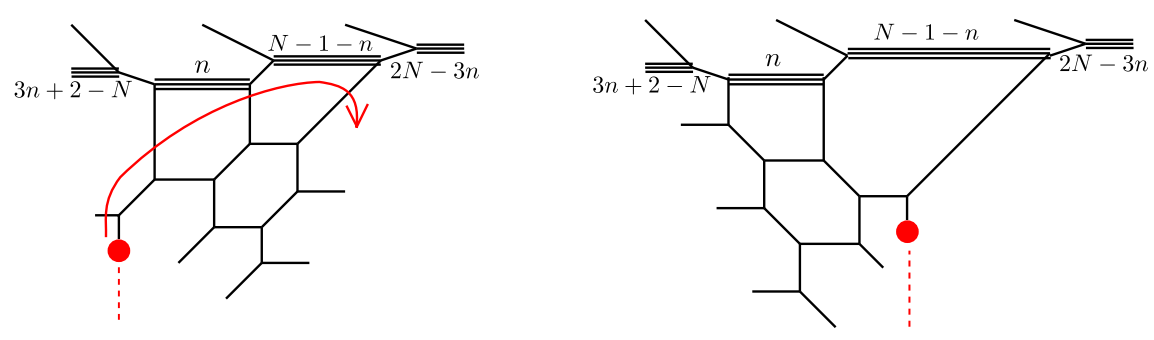

Figure 16. Derivation of the distribution duality 2.
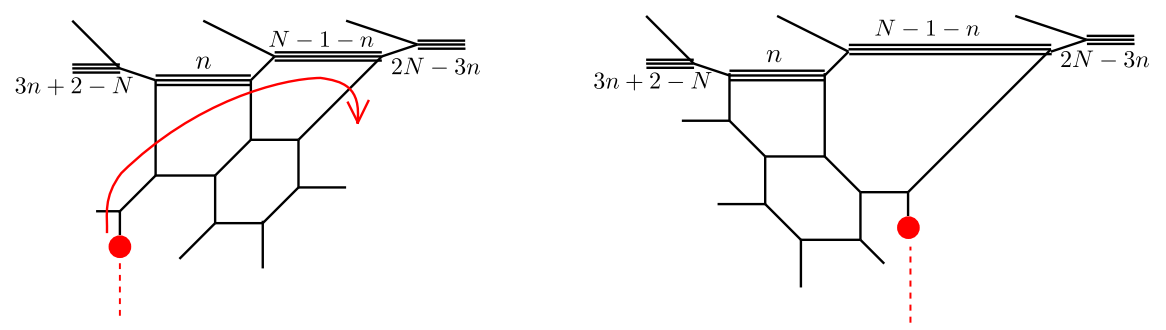

Figure 17. Derivation of the distribution duality 3 .

Contrary to the previous case, they are not related by shifting $n$ any more. However, this duality keeps the total number of flavors, total number of gauge ranks, and total number of CS levels. In this sense, this is also the analogue of the distribution duality.

It would be also straightforward to see the following duality from figure 17:

- $5 \mathrm{~d}[3 n+3-N]-\mathrm{SU}(n+1)_{0}-\mathrm{SU}(N+1-n)_{-1}-[2 N-3 n+1]$

- $5 \mathrm{~d}[3 n+4-N]-\mathrm{SU}(n+2)_{-1}-\mathrm{SU}(N-n)_{0}-[2 N-3 n]$

It would be also possible to combine them with the flavor decoupling limit to move $n_{1}$ and $n_{2}$ flavor branes upward. Therefore, the discussion here can be summarized as the following duality:

- $5 \mathrm{~d}\left[3 n+3-N-n_{1}\right]-\mathrm{SU}(n+1)_{n_{1}}-\mathrm{SU}(N+1-n)_{n_{2}-\frac{k}{2}}-\left[2 N-3 n+3-k-n_{2}\right]$

- $5 \mathrm{~d}\left[3 n+6-N-k-n_{1}\right]-\mathrm{SU}(n+2)_{n_{1}-\frac{k}{2}}-\mathrm{SU}(N-n)_{n_{2}}-\left[2 N-3 n-n_{2}\right]$

for $k=0,1,2$.

Although the processes to move the [0,1] 7-brane as in figure 15, 16, or 17 may look exotic, it looks often quite natural when we see this in the S-dual frame. Take figure 17 as an example and consider the S-transformation for both. Then, we observe that both diagrams correspond to the identical $5 \mathrm{~d}$ theory in the form

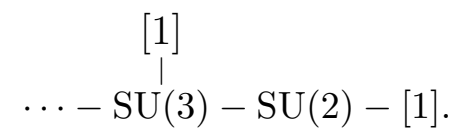

The deformation of moving the [0,1] 7-brane in figure 17 is translated to moving the D7-brane. This D7-brane corresponds to the flavor charged under the SU(3) gauge group 

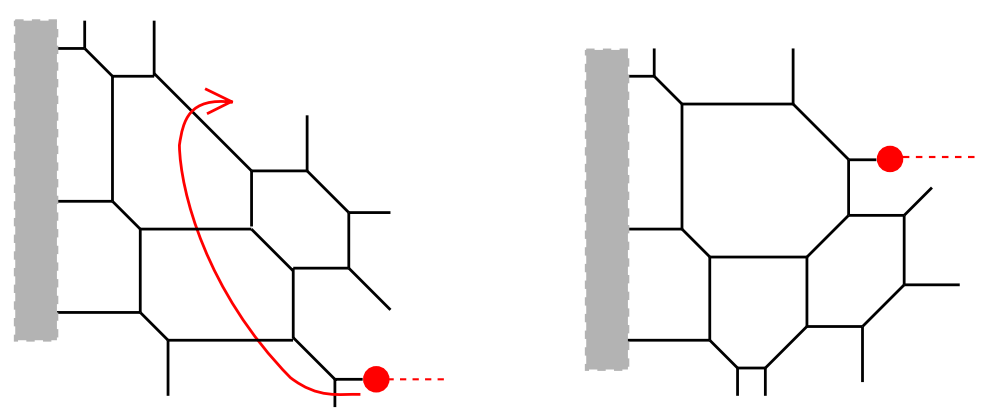

Figure 18. Distribution duality in the S-dual frame

and moving it simply corresponds to changing the mass of this flavor. Therefore, the "distribution duality", which change the distribution of the rank of the gauge group in the original frame, is actually just the mass deformation in the S-dual frame. This is essentially the same observation seen in section 3.4 that depending on the value of the mass parameters of the flavors, the same $5 \mathrm{~d}$ gauge theory can be mapped to different gauge theories under the identical S-duality transformation.

\subsection{Chain of duality and exotic example}

Combining what we studied in section 3.4 and section 3.5 , we demonstrate that varous $5 \mathrm{~d}$ quiver gauge theories have identical $6 \mathrm{~d}$ UV fixed point.

We again consider the $5 \mathrm{~d}$ theories corresponding to $6 \mathrm{~d} \mathrm{SU}(6)$ with $N_{f}=14$ and $N_{a}=1$ and with a tensor multiplet. In figure 19, we write various web diagrams, which are related to each other either by mass deformation or the distribution duality, which is sometimes interpreted also as the mass deformation in the S-dual frame.

In the original frame, we see that

$$
\begin{aligned}
\text { I, II, III }:[6]-\mathrm{SU}(4)_{0}-\mathrm{SU}(4)_{0}-[6] \\
\text { IV, V, VI }:[9]-\mathrm{SU}(5)_{0}-\mathrm{SU}(3)_{0}-[3] \\
\text { VII, VIII, IX, X }:[12]-\mathrm{SU}(6)_{0}-\mathrm{SU}(2) \\
\text { XI, XII }:[12]-\mathrm{SU}(7)_{\frac{3}{2}} ?-" \mathrm{SU}(1)^{\prime \prime}
\end{aligned}
$$

The last one includes "SU(1)" factor and thus, does not have the standard field theory description and moreover, does not look fit with the distribution duality discussed previously. However, by using the analogous process to move one of the [0,1] 7-brane as in figure 15,16 , or 17 , we can show that diagram $X$ and diagram $X I$ are actually connected by such process. We note that II $\leftrightarrow$ IV and $\mathrm{VI} \leftrightarrow \mathrm{VIII}$ are also related by the distribution dualities. 

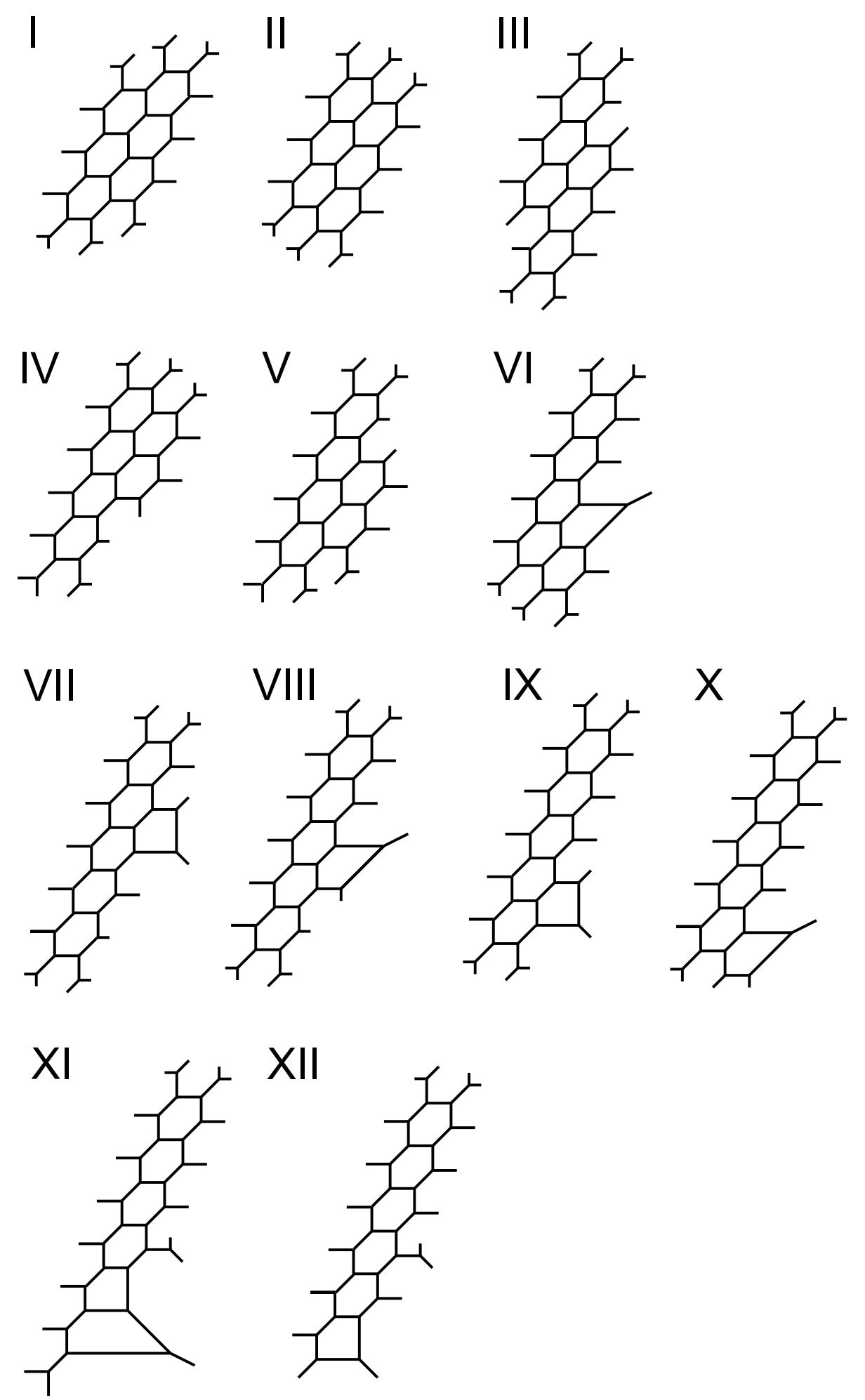

Figure 19. Chain of dualities. 
In the S-dual frame, the gauge theory description is possible only for the following:

$$
\begin{gathered}
\text { I }:[5]-\mathrm{SU}(3)_{0}-\mathrm{SU}(3)_{0}-\mathrm{SU}(3)_{0}-[5] \\
{[1] \quad[1]} \\
\text { II, IV }:[3]-\mathrm{SU}(2)-\mathrm{SU}(3)_{0}-\mathrm{SU}(3)_{0}-\mathrm{SU}(2)-[3] \\
\text { III }:[3]-\mathrm{SU}(2)-\mathrm{SU}(2)-\mathrm{SU}(3)_{0}-\mathrm{SU}(2)-\mathrm{SU}(2)-[3] \\
\text { V }:[3]-\mathrm{SU}(2)-\mathrm{SU}(2)-\mathrm{SU}(3)_{\frac{1}{2}}-\mathrm{SU}(3)_{0}-[5] \\
\text { VII }:[3]-\mathrm{SU}(2)-\mathrm{SU}(2)-\mathrm{SU}(3)_{1}-\mathrm{SU}(2)-\mathrm{SU}(2)-[3] \\
\text { VIII }:[3]-\mathrm{SU}(2)-\mathrm{SU}(2)-\mathrm{SU}(2)-\mathrm{SU}(2)-\mathrm{SU}(3)_{1}-[3] \\
\quad[1] \\
\text { XII }:[3]-\mathrm{SU}(2)-\mathrm{SU}(2)-\mathrm{SU}(2)-\mathrm{SU}(2)-\mathrm{SU}(2)-\mathrm{SU}(2)
\end{gathered}
$$

We omitted the case where the diagram does not have clear field theory interpretation. In this way, we obtain various $5 \mathrm{~d}$ quiver gauge theories which have identical $6 \mathrm{~d}$ UV fixed point.

Here, we comment on the global symmetry. These theories are all expected to have SU(14) global symmetry at UV fixed point. Indeed, we can explicitly check that 7-brane monodromy analysis for these diagrams all show this expected global symmetry. When we apply the method [31] using instanton operators, some of these nicely shows affine SU(14) symmetry and thus, consistent with the claim that these $5 \mathrm{~d}$ theories have $6 \mathrm{~d}$ UV fixed point with SU(14) global symmetry. However, strange to say, application of [31] to some of these $5 \mathrm{~d}$ quiver gauge theories fails to reproduce the expected affine structure. For example, the S-dual description of diagram III, the central gauge node SU(3) has flavor $N_{f}<2 N$ and thus, does not show affine symmetry. We claim that one-instanton analysis is not enough in this case. It would be interesting to generalize the method with instanton operators to include the higher instanton contribution for these case to check the expected affine global symmetry is obtained.

\section{$45 \mathrm{~d}$ dualities from 6d: generalization}

In this section, we consider a general 6d quiver theory constructed by NS5-branes, D6branes, D8-branes an $08^{-}$-plane introduced in $[28,29]$. It is straightforward to repeat essentially the same analysis done in section 2 and 3 for the general $6 \mathrm{~d}$ quiver theories. We will see that the $5 \mathrm{~d}$ reduction yields various $5 \mathrm{~d}$ dualities.

\section{$4.1 \quad 6 \mathrm{~d} \mathrm{Sp}-\prod \mathrm{SU}$ quiver theories}

We first focus on $6 \mathrm{~d}$ quiver theories with one $S p$ gauge node, which is a generalization of the $6 \mathrm{~d}$ quiver considered in section 2 . The simple generalization of the setup in section 2 realizes a $6 \mathrm{~d}$ linear quiver

$$
6 d \mathrm{Sp}(N)-\mathrm{SU}(2 N+8)-\mathrm{SU}(2 N+16)-\cdots-\mathrm{SU}(2 N+8(n-1))-[2 N+8 n],
$$

and its Type IIA brane configuration is given in figure 20. The case with $n=1$ falls in to the case in section 2 . Hence, we concentrate on the cases with $n>1$. The global symmetry 


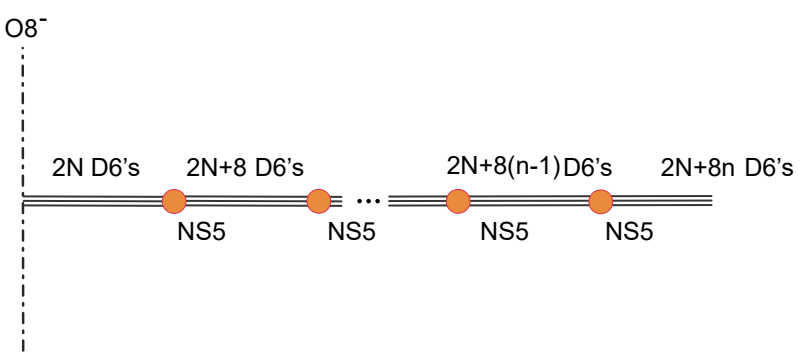

Figure 20. Type IIA brane configuration for the $6 \mathrm{~d}$ linear quiver theory (4.1).

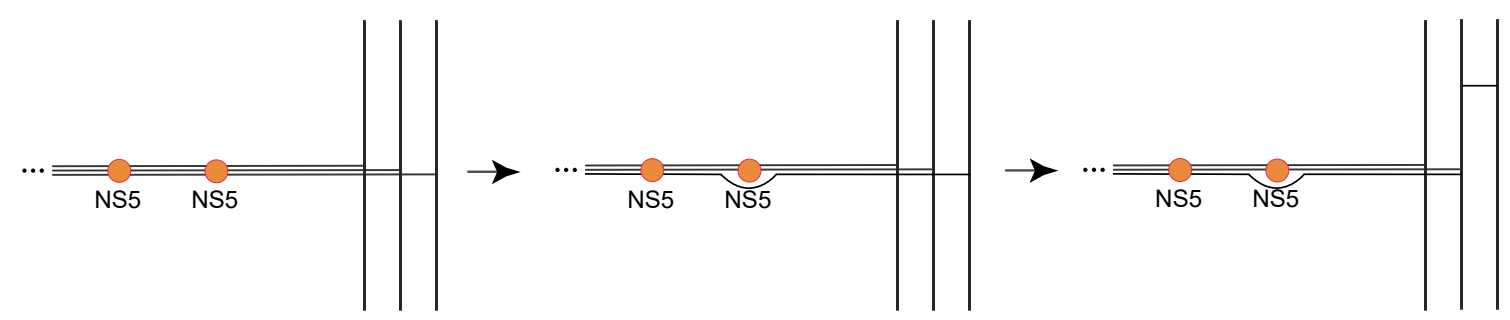

Figure 21. A process of the 6d Higgsing.

is generically $\mathrm{SU}(2 N+8 n) \times \mathrm{U}(1)$. The number of the tensor multiplets is $n$ and the total rank of the gauge groups is $N(2 n-1)+4 n^{2}-5 n+1$.

We can further generalize the $6 \mathrm{~d}$ quiver theory by Higgsing. The flavor symmetry of the brane construction of the canonical $6 \mathrm{~d}$ quiver (4.1) is associated to a symmetry on semi-infinite $2 N+8 n$ D6-branes at the end. It is also possible to let one semi-infinite D6brane become finite and end on one D8-brane. Since each D6-brane end on one D8-brane, we have $2 N+8 \mathrm{D} 8$-branes in total. In this picture, each D6-brane at the end connect an NS5-brane with a D8-brane. A Higgs branch of the 6d theory arises by attaching one D6brane on two or more D8-branes. Since the condition of preserving supersymmetry implies that only one D6-brane can connect an NS5-brane with a D8-brane [40], the D6-brane should connect to other D6-brane between NS5-branes by jumping some NS5-branes as in figure 21. Then, there appears fractionated D6-branes between D8-branes. The motion of the D6-branes between D8-branes describes the vev of the corresponding hypermultiplets. After sending the fractionated D6-branes into infinity, we have decoupled D8-branes and hence the flavor symmetry is reduced. Due to the appearance of the jumping of D6-branes over NS5-branes, the ranks of some gauge groups are also reduced.

In general, such a Higgsing is classified by a Young diagram as in [2]. When one represents a Young diagram by a vector with non-increasing numbers, each component represent the number of D6-branes ending on D8-brane. The total number of the boxes in the Young diagram is $2 N+8 n$. Therefore, the brane configuration in figure 20 corresponds to a Young diagram $[1, \cdots, 1]$ with $2 N+8 n$ entries of 1's. A general Higgsing corresponds to a Young diagram $[n, \cdots, n, n-1, \cdots, n-1, \cdots, 2, \cdots, 2,1, \cdots, 1]$ where the number of $l$ is $k_{l}$ with a condition $\sum_{l=1}^{n} l \cdot k_{l}=2 N+8 n$. The 6 d gauge theory content can be read off by moving D8-branes to the left in the brane configuration until no D6-branes end on 


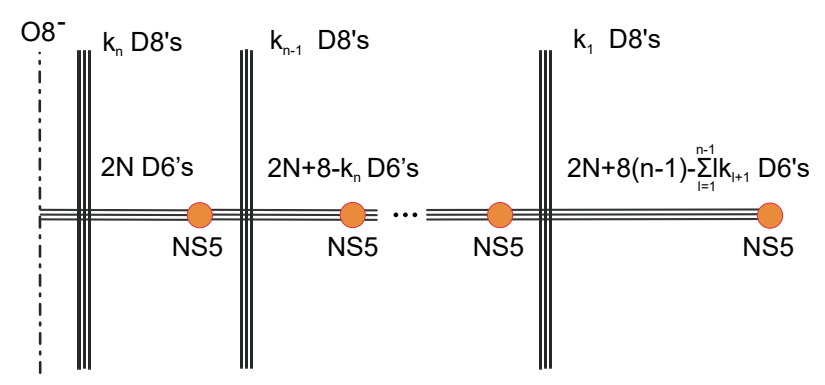

Figure 22. Type IIA brane configuration for the $6 \mathrm{~d}$ linear quiver theory (4.2).

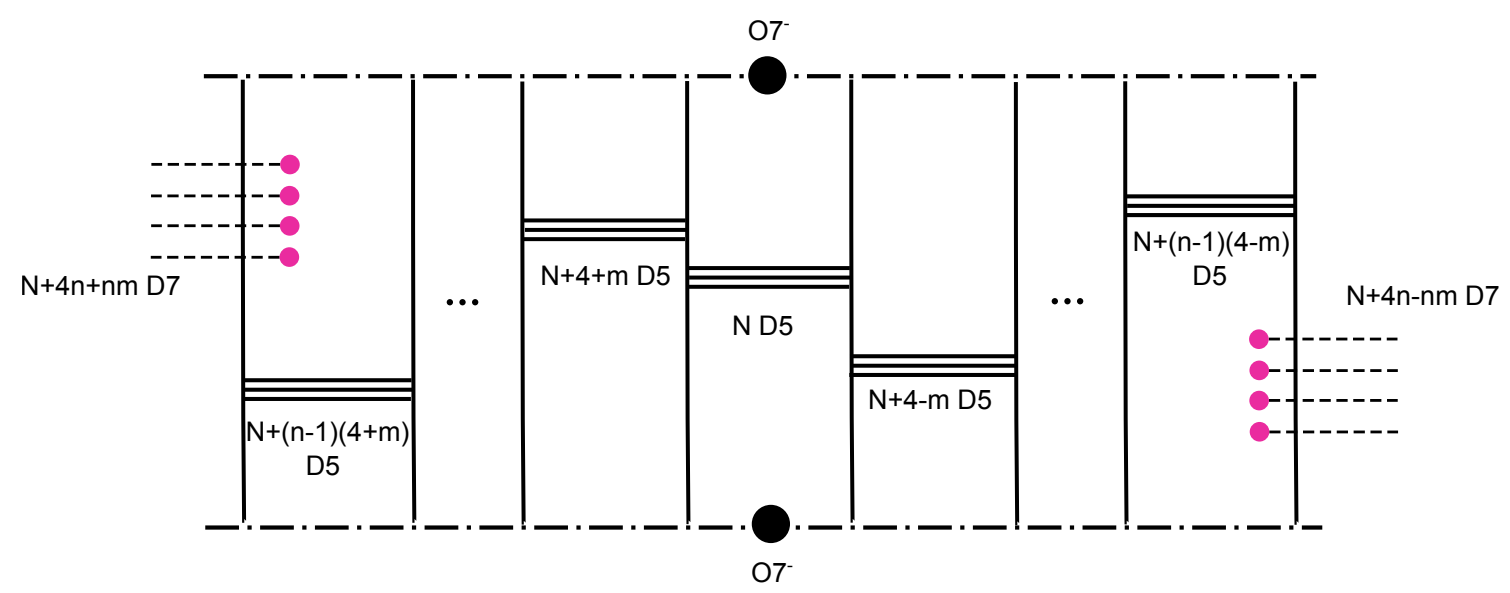

Figure 23. Type IIB brane configuration after performing a T-duality to figure 20 .

the D8-branes. Depending on the Young diagram, various fundamental hypermultiplets are introduced to some middle gauge nodes in the $6 \mathrm{~d}$ quiver. Hence, after a Higgsing corresponds to the Young diagram $[n, \cdots, n, n-1, \cdots, n-1, \cdots, 2, \cdots, 2,1, \cdots, 1]$, we obtain the following $6 \mathrm{~d}$ quiver theory at low energies

$$
\begin{array}{ccc}
{\left[k_{n}\right]} & {\left[k_{n-1}\right]} & {\left[k_{1}\right]} \\
\mid & \mid & \\
\mid & & \\
6 d \mathrm{Sp}(N) & -\mathrm{SU}\left(2 N+8-k_{n}\right)-\cdots-\mathrm{SU}\left(2 N+8(n-1)-\sum_{l=1}^{n-1} l k_{l+1}\right) .
\end{array}
$$

Type IIA brane configuration is depicted in figure 22 .

We will consider a circle compactification of the $6 \mathrm{~d}$ quivers of (4.1) and (4.2), and see the $5 \mathrm{~d}$ descriptions of the theories as well as the $5 \mathrm{~d}$ dualities.

\subsubsection{5d SU quviers}

We first focus on an $S^{1}$ compactification of the canonical 6d quiver theory (4.1). The Tduality along the $S^{1}$ gives us a 5-brane configuration with two $O 7^{-}$-planes as in figure 23 . After the T-duality, we have the distribution ambiguity discussed in section 3.1. The number of D5-branes in the middle column is always $N$, which corresponds to the first gauge node of the $6 \mathrm{~d}$ quiver 4.1. The $N+8$ color D5 branes originated from the 2 nd gauge 


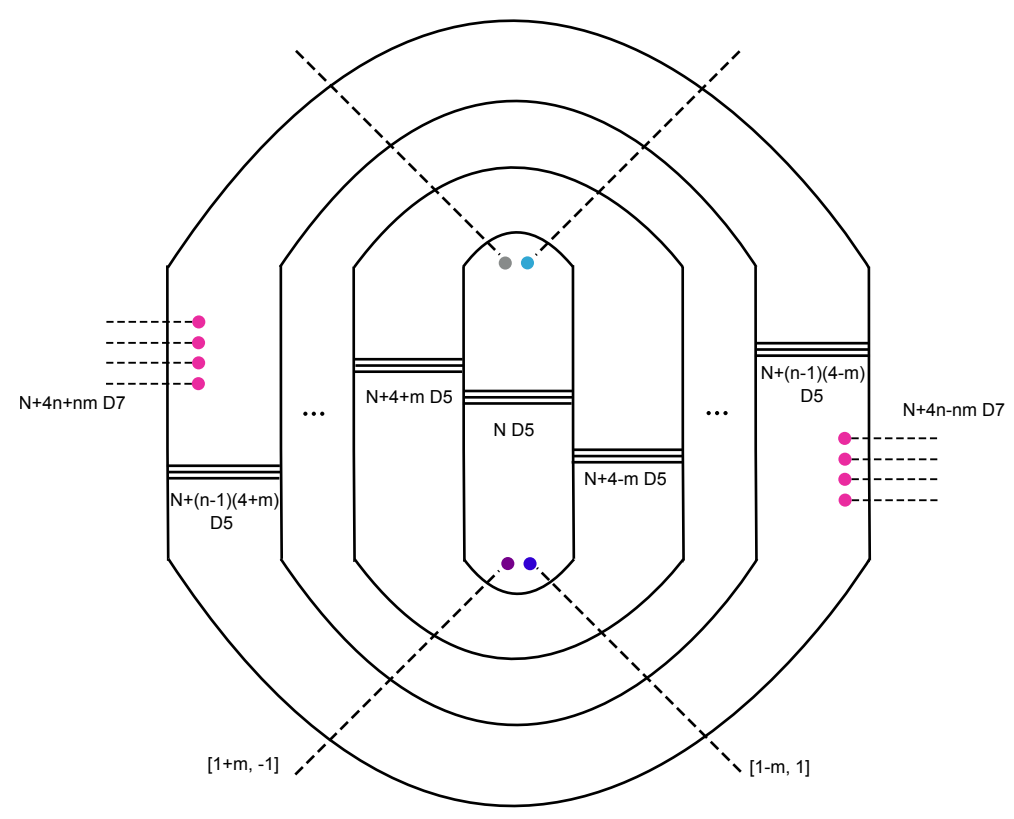

Figure 24. The 5-brane web diagram after splitting two $O 7^{-}$-planes compared to figure 23. Note that the slope of the lines is schematically depicted and does not represent the corresponding 5-brane charge precisely.

node in $6 \mathrm{~d}$ quivers 4.1 are distributed into the next columns, which are left and right to the center, respectively. The number of the D5-branes in these columns can change by the distribution ambiguity. We label the ambiguity by a non-negative number $m$, and set the number of the D5-branes in the left column to the middle to be $N+4+m$ and the number of the D5-branes in the right column to the middle to be $N+4-m$.

The $N+8 i$ color $\mathrm{D} 5$ branes corresponding to the $(i+1)$-th gauge node in 6 d quivers 4.22 are distributed into the $i$-th left columns and $i$-th right columns from the center. Here, we need to care about the condition that the resulting 5-brane web diagram after pulling all the 7-branes outside has a $5 \mathrm{~d}$ gauge theory interpretation. In fact, this condition may determine the number of D5-branes in the column next to the column which is next to the middle one. By repeating this analysis, it turns out that there is only one degree of freedom for the ambiguity parameterized by $m$. The resulting distribution is depicted in figure 23 .

Next task is the resolution of the $O 7^{-}$-planes. As in section 2, we can consider either splitting both $\mathrm{O}^{-}$-planes or splitting only one of the two $O 7^{-}$-planes. We consider the former case first. The condition that the final 5-brane web after pulling out all the 7-branes should have a $5 \mathrm{~d}$ gauge theory interpretation also constrains the splitting type of the $\mathrm{O}^{-}$planes. The relative difference between the two splitting type is not important, and hence we fix the splitting type of the upper $O 7^{-}$-plane to be $\mathbf{B} \mathbf{C}$. Then, the splitting type of the lower $O 7^{-}$-plane is determined to be $\mathbf{X}_{[1+m,-1]} \mathbf{X}_{[1-m, 1]}$ in order that the resulting 5-brane web diagram after pulling all the 7-branes outside has a 5 d gauge theory interpretation. The resolution creates the $n 5$-brane loops as in figure 24 . Then, we move the branch cuts of $\mathbf{B}, \mathbf{C}$ and $\mathbf{X}_{[1+m,-1]} \mathbf{X}_{[1-m, 1]}$ so that some of the D5-branes and D7-branes cross the branch cuts 


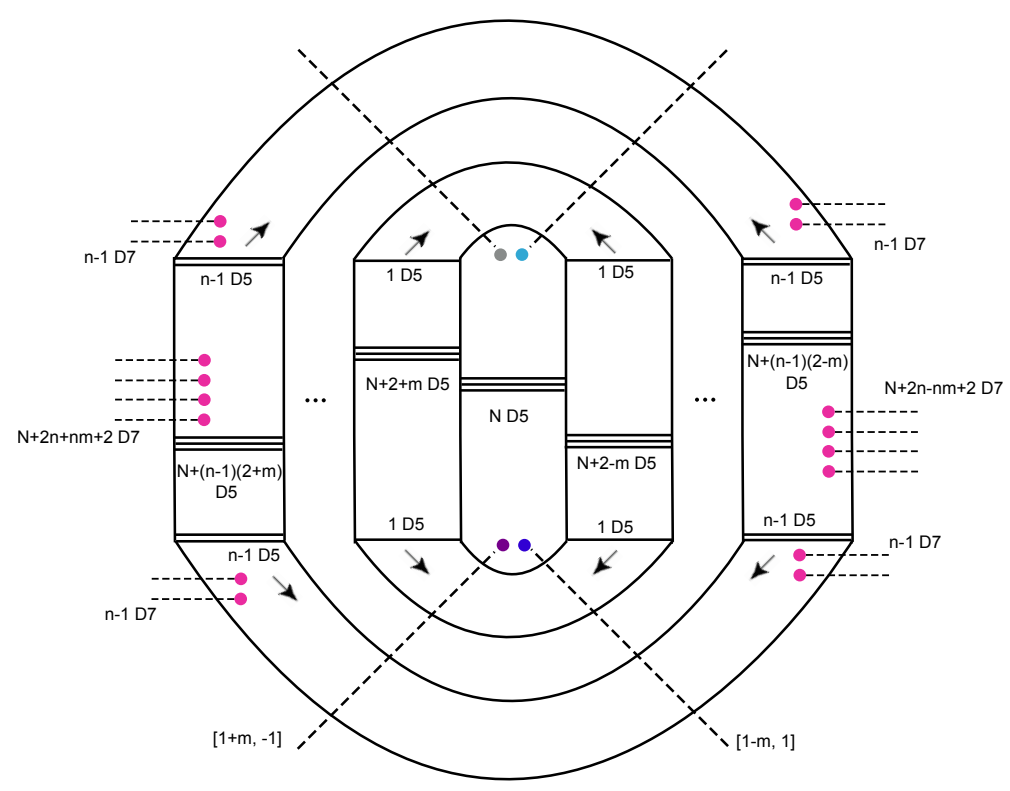

Figure 25. The motion of the branch cuts of $\mathbf{B}, \mathbf{C}$ and $\mathbf{X}_{[1+m,-1]} \mathbf{X}_{[1-m, 1]}$. Relatively, it can be realized by moving some amount of D5-branes and D7-branes. The D5-branes and the D7-branes that move are indicated by arrows in this figure.

as in figure 25. In the upper part of the diagram, D7-branes and D5-branes cross the branch cuts of the $\mathbf{B}$ and $\mathbf{C}$ 7-branes in a counterclockwise and clockwise direction respectively. Then the D7-branes become [0,1] 7-branes, and the D5-branes become NS5-branes. On the other hand, in the lower part of the diagram, D7-branes and D5-branes cross the branch cuts of the $\mathbf{X}_{[1+m,-1]} \mathbf{X}_{[1-m, 1]}$ 7-branes in a counterclockwise and clockwise direction respectively. Then, the D7-branes and the D5-branes become $[m,-1]$-brane and $(m,-1)$ 7-brane respectively. After crossing the branch cuts, the 5 -brane loops are divided by $2 n$ vertical lines as in figure 26 . This is the origin of $5 \mathrm{~d}$ quiver theories with $2 n-1$ gauge nodes.

After pulling out all the 7-branes, the 5-brane web diagram becomes the one in figure 27 . The $5 \mathrm{~d}$ gauge theory realized by the 5 -brane web is then

$$
\begin{gathered}
{\left[L_{1}\right]} \\
\quad \mid \\
\mathrm{SU}\left(N_{1}\right)-\mathrm{SU}\left(N_{2}\right)-\cdots-\mathrm{SU}\left(N_{n-1}\right)-\mathrm{SU}(2 N+2 n)-\mathrm{SU}\left(M_{n-1}\right)-\cdots-\mathrm{SU}\left(M_{2}\right)-\mathrm{SU}\left(M_{1}\right) \\
\mid
\end{gathered}
$$

where

$$
\begin{aligned}
N_{l} & =N+2 n+(n-l) m, \quad \text { for } \quad 1 \leq l \leq n-1, \\
M_{l} & =N+2 n-(n-l) m, \quad \text { for } \quad 1 \leq l \leq n-1, \\
L_{1} & =N+2 n+2+n m, \\
R_{1} & =N+2 n+2-n m .
\end{aligned}
$$

The parameters are constrained such that the rank of each gauge group or the number of the flavors should be greater than zero at least. 


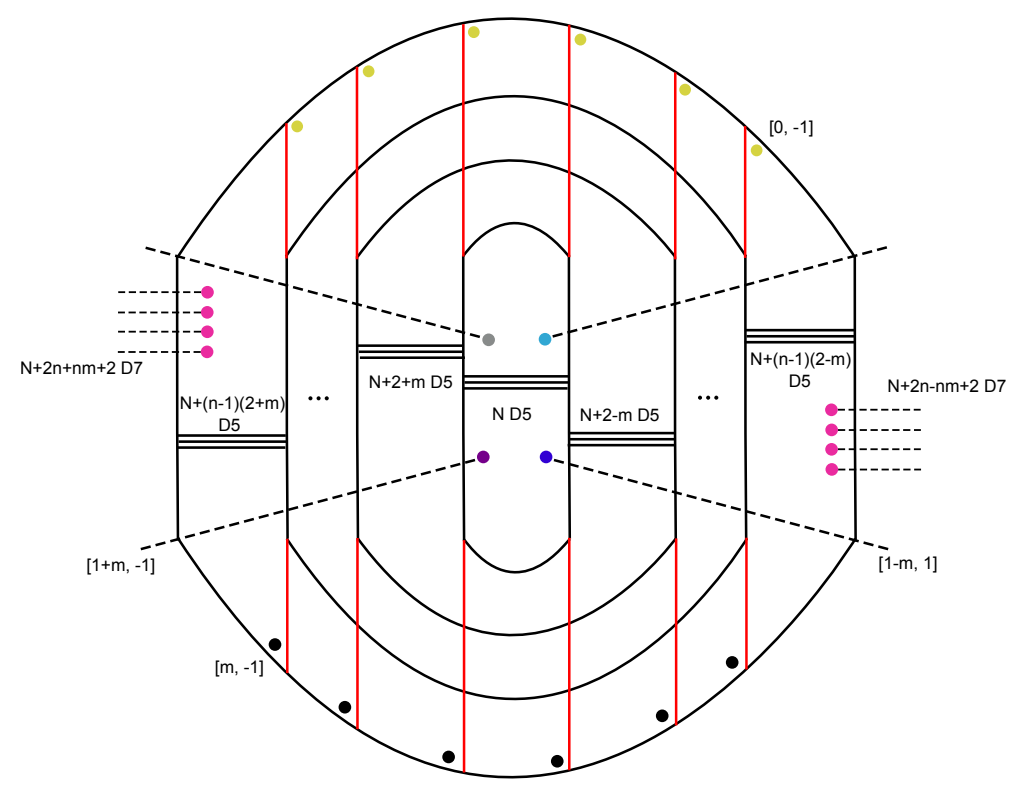

Figure 26. The schematic diagram after moving the branch cuts of $\mathbf{B}, \mathbf{C}$ and $\mathbf{X}_{[1+m,-1]} \mathbf{X}_{[1-m, 1]}$ 7-branes from figure 24 . The red lines arise from D5-branes which cross the branch cuts of $\mathbf{B}, \mathbf{C}$ and $\mathbf{X}_{[1+m,-1]} \mathbf{X}_{[1-m, 1]}$ 7-branes. The [0,1] 7-branes denoted by the yellow circles in the upper part of the diagram arise from D7-branes which cross the branch cuts of $\mathbf{B}, \mathbf{C} 7$-branes. On the other hand, the $[m,-1]$ 7-branes denoted by the black circles in the lower part of the diagram arise from D7-branes which cross the branch cuts of $\mathbf{X}_{[1+m,-1]} \mathbf{X}_{[1-m, 1]}$ 7-branes.

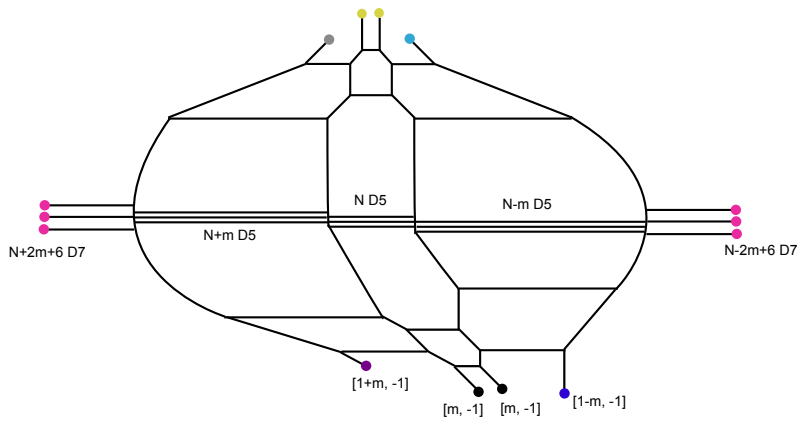

Figure 27. The 5-brane web diagram after pulling out all the 7-branes outside of the 5-brane loops from the one in figure 25. For simplicity, we write a web diagram of $n=2$.

The number of the Coulomb branch moduli can be easily counted and it is $(2 n-$ 1) $(N+2 n-1)$ which is the sum of the number of the tensor multiplets and the number of the vector multiplets in the Cartan subalgebra of the 6d quiver theory (4.1). The global symmetry analysis by 7 -branes should recover $\mathrm{SU}(2 N+8 n)$ since the very first 5 -brane web gives $2 N+8 n$ D7-branes on top of each other. ${ }^{7}$

The different choice of $m$ in (4.3) gives a different-looking 5 d gauge theory. However, we claim that they are dual to each other, and it is the distribution duality.

\footnotetext{
${ }^{7}$ Note that a D7-brane is mutually local to an $O 7^{-}$-plane. D7-branes do not change its change when it crosses the branch cut of an $O 7^{-}$-plane.
} 


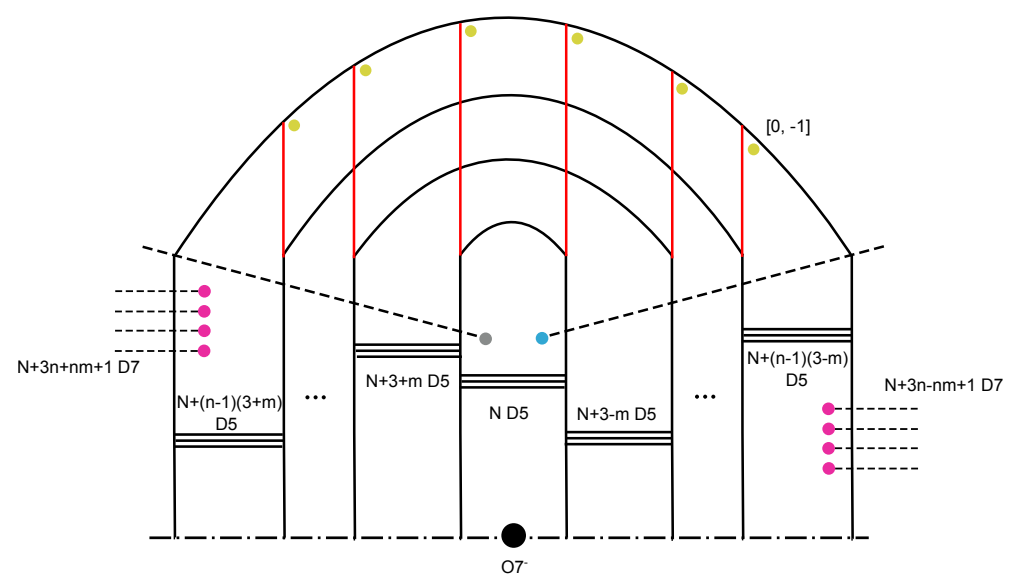

Figure 28. The schematic diagram after moving the branch cuts of $\mathbf{B}, \mathbf{C}$ and $\mathbf{X}_{[1+m,-1]} \mathbf{X}_{[1-m, 1]}$ 7-branes in the case when one resolves one of the $\mathrm{O}^{-}$-planes in figure 23. The motion corresponds to the upper ones depicted in figure 25 .

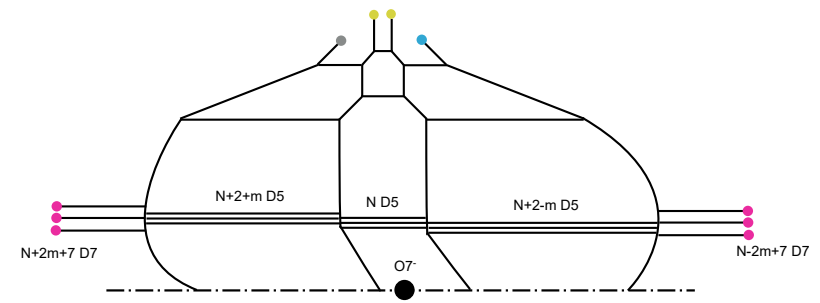

Figure 29. The 5-brane web diagram realizing the $5 \mathrm{~d} \mathrm{Sp}(N+2)-\mathrm{SU}(2 N+8)-[2 N+14]$. We only write the web diagram for the case with $n=2$ for simplicity. This web diagram can be obtained by pulling all the 7 -branes outside the 5 -brane loops from the configuration depicted in figure 28 with $n=2$.

\subsection{2 $5 \mathrm{~d} \mathrm{Sp}-\prod \mathrm{SU}$ quivers}

We then consider the case of splitting only one of the two $O 7^{-}$-planes. The quantum resolution splits the $\mathrm{O}^{-}$-plane into $\mathbf{B}$ and $\mathbf{C}$ 7-branes. The analysis is essentially the same as in section 4.1.1. About the motion of the branch cuts of 7-branes, we can only consider the one corresponding to the upper transformations in figure 25, the resulting 5 -brane configuration is depicted in 28 .

After pulling out all the 7-branes, the final 5-brane web diagram is given by figure 29 . Note that in this case the distribution ambiguity does not matter. Due to the presence of the $O 7^{-}$-plane, the column which originally has $N+k(4+m)$ D5-branes is connected to the column which originally has $N+k(4-m)$ D5-branes for $1 \leq k \leq n-1$. Therefore, the resulting $5 \mathrm{~d}$ theory is

$$
\mathrm{Sp}(N+n)-\mathrm{SU}(2 N+2 n+4)-\mathrm{SU}(2 N+2 n+8)-\cdots-\mathrm{SU}(2 N+6 n-4)-[2 N+6 n+2] .
$$

Due to the process of obtaining (4.8), the 7-brane analysis should give the $\mathrm{SU}(2 N+8 n)$ flavor symmetry. The number of the Coulomb branch moduli is again $(2 n-1)(N+2 n-1)$, 
which is consistent with the sum of the number of the tensor multiplets and the number of the vector multiplets in the Cartan subalgebra of the $6 \mathrm{~d}$ quiver theory (4.1).

\subsubsection{5d dualities}

From the same $6 \mathrm{~d}$ quiver theory of (4.1), we have obtained two types of $5 \mathrm{~d}$ quiver theories. One type has only $S U$ gauge nodes and the other type has one $S p$ gauge node with other $S U$ gauge nodes. Those two theories are described by essentially the same 5-brane web diagrams and we claim that they are dual to each other. This is the generalization of the claim in section 2.3 .

As in section 2.4, it is possible to generate further dual $5 \mathrm{~d}$ theories by acting the $\mathrm{SL}(2, \mathbb{Z})$ duality on (4.3). For example, let us consider a case where $m=0$, which yields,

$$
[N+2 n+2]-\mathrm{SU}(N+2 n)-\cdots-\mathrm{SU}(N+2 n)-\cdots-\mathrm{SU}(N+2 n)-[N+2 n+2],
$$

where it has $2 n-1$ gauge nodes. The S-duality or the 90 degrees rotation gives

$$
[2 n+2]-\mathrm{SU}(2 n)-\cdots-\mathrm{SU}(2 n)-\cdots-\mathrm{SU}(2 n)-[2 n+2],
$$

where it has $N+2 n-1$ gauge nodes. It is in fact more interesting to see the TST-duality or the 45 degrees rotation. The resulting theory is

$$
\begin{gathered}
{[3]} \\
\mid[1] \\
\mathrm{SU}(2)-\mathrm{SU}(3)-\cdots-\mathrm{SU}(N+2 n)-\mathrm{SU}(N+2 n)- \\
{[1]} \\
\cdots-\mathrm{SU}(N+2 n)-\mathrm{SU}(N+2 n)-\cdots-\mathrm{SU}(3)-\mathrm{SU}(2),
\end{gathered}
$$

where it has $2 n-3 \mathrm{SU}(N+2 n)$ gauge nodes. In particular, when $n=2$, the 5 d quiver (4.11) has a peculiar form

$$
\begin{array}{cc}
{[3]} & {[2]} \\
\mid & {[3]} \\
\mathrm{SU}(2) & -\mathrm{SU}(3)-\cdots-\mathrm{SU}(N+2 n)-\cdots-\mathrm{SU}(3)-\mathrm{SU}(2),
\end{array}
$$

which can be regarded as gauging $\mathrm{SU}(N+2 n)$ in the $\mathrm{SU}(N+2 n+2)$ flavor symmetry of two the $T_{N+2 n}$ Tao theories which we will discuss in detail in section 6 .

As in section 2, it is possible to decouple some flavors from the $5 \mathrm{~d}$ theories whose UV completion is the $6 \mathrm{~d}$ SCFT. After decoupling some flavors, the $5 \mathrm{~d}$ theory has a $5 \mathrm{~d}$ UV fixed point. Suppose we have dual $5 \mathrm{~d}$ theories whose UV completion is the same 6d SCFT, decoupling the same flavors in the both theories leads to another dual $5 \mathrm{~d}$ theories whose UV completion is a same 5d SCFT. Working on explicit examples is quite straightforward and can be done in parallel to the analysis in section 2.3 and 2.4.

\subsubsection{Higgsed cases}

Let us then move on to a circle compactification of the Higgsed 6d quiver theories (4.2). After performing the T-duality along the $S^{1}$, we again obtain a brane configuration with 


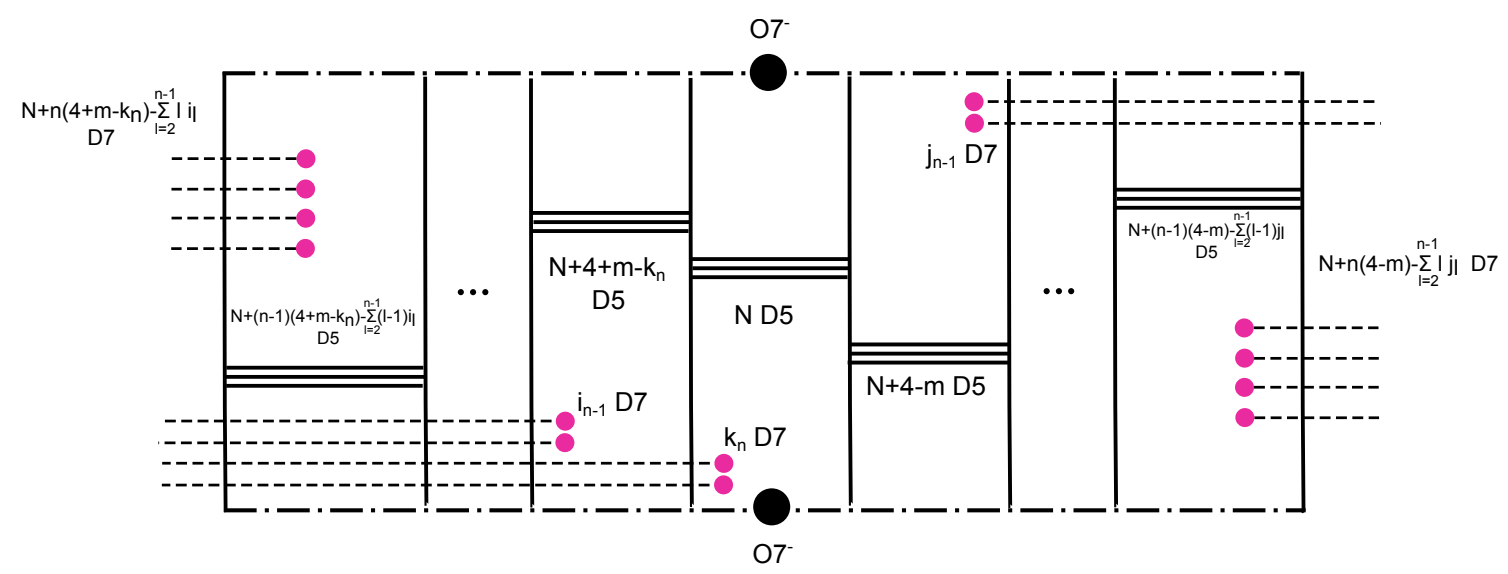

Figure 30. Type IIB brane configuration after performing a T-duality to figure 22 . We defined non-negative numbers $i_{l}, j_{l}$ such that they satisfy $i_{l}+j_{l}=k_{l}$ for $l=1, \cdots, n-1$.

5-branes and two $O 7^{-}$-planes. The difference from the cases in section 4.1.2 is that we now have D7-branes in various columns, depending on the number of the fundamental hypermultiplets attached to some gauge nodes in the $6 \mathrm{~d}$ quiver theory (4.2). In this case, there is another ambiguity of distributing D7-branes in addition to the distribution of D5branes which we saw in section 4.1.1. Due to this distribution ambiguity of D7-branes, one can allocate $k_{n-l}$ D7-branes for $1 \leq i \leq n-1$, which originate from the $k_{n-l}$ D8-branes in the Type IIA brane configuration in figure 22, to the $i$-th left column and the $i$-th right column from the center column. Again, the number of D7-branes in the middle column is fixed to be $k_{n}$. We choose the branch cuts of D7-branes in the columns left from the center extend in the left direction, and the branch cuts of D7-branes in the columns right from the center extend in the right direction. Furthermore, we also assume that the branch cuts of $k_{n}$ D7-branes extend in the left direction.

The requirement that the final 5 -brane web diagram admits a $5 \mathrm{~d}$ gauge theory interpretation constrains the number of D5-branes in each column except for the center. The number of D5-branes in the middle column is again always $N$. Compared to the case in section 4.1.2, we have the branch cuts of D7-branes in the columns that extend in either left or right direction. The branch cuts also affect the number of D5-barnes in each column which gives a web diagram admitting a 5 d gauge theory description at the final stage after pulling all the 7 -branes outside. It turns out that the 5 -brane configuration depicted in figure 30 yields a 5 -brane web which admits a $5 \mathrm{~d}$ gauge theory interpretation.

In order to go from the brane configuration in figure 30 to a 5 -brane web yielding a $5 \mathrm{~d}$ gauge theory, we take two steps as in section 4.1.1 and 4.1.2. The first step is we resolve either two or one of the $O 7^{-}$-planes. When we split two $O 7^{-}$-planes, we fix the splitting type of the upper $\mathrm{O7}^{-}$-plane into $\mathbf{B}$ and $\mathbf{C} 7$-branes, and the splitting type of the lower $O 7^{-}$-plane is chosen to be $\mathbf{X}_{[1+m,-1]} \mathbf{X}_{[1-m, 1]}$ so that the final 5-brane web admits a $5 \mathrm{~d}$ gauge theory interpretation. When we split only one of the two $O 7^{-}$-planes, the splitting type may be generically $\mathbf{B}$ and $\mathbf{C}$ 7-branes. The second step is moving the branch cuts of 7-branes which arise by the quantum resolution of two or one $\mathrm{O}^{-}-$-plane. After the second 


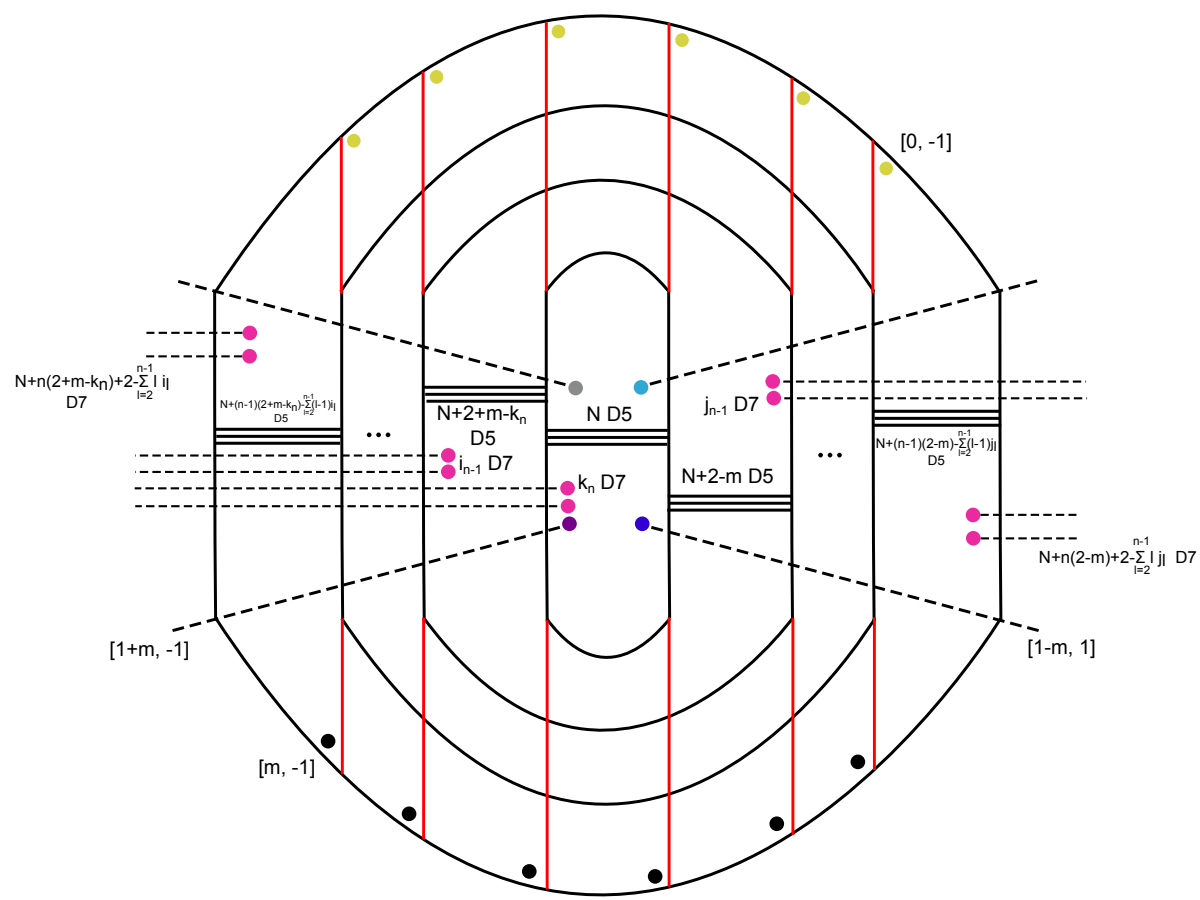

Figure 31. Type IIB brane configuration after the two steps from the one in figure 30 when two $\mathrm{O}^{-}$-planes are resolved.

step, the 5-brane loops are divided by vertical lines and the structure eventually leads to a $5 \mathrm{~d}$ quiver theory. The procedure is completely parallel to the one in section 4.1.1 and 4.1.2.

When we split the two $\mathrm{O}^{-}$-planes, we obtain the 5-brane configuration in figure 31 after the two steps. After pulling all the 7-branes from figure 31, the final 5-brane web configuration gives rise to a 5 d quiver theory with $S U$ gauge nodes with various flavors attached to each node. The explicit expression is

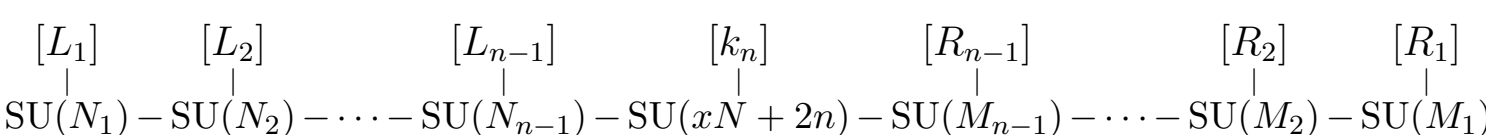

where

$$
\begin{array}{ll}
N_{p}=N+2 n+(n-p)\left(m-k_{n}\right)-\sum_{l=p+1}^{n-1}(l-p) i_{l} & \text { for } \quad 1 \leq p \leq n-1, \\
M_{p}=N+2 n-(n-p) m-\sum_{l=p+1}^{n-1}(l-p) j_{l} & \text { for } \quad 1 \leq p \leq n-1, \\
L_{p}=i_{p}, & \text { for } \quad 2 \leq p \leq n-1, \\
L_{1}=N+2 n+n\left(m-k_{n}\right)-\sum_{l=2}^{n-1} l i_{l}+2, &
\end{array}
$$




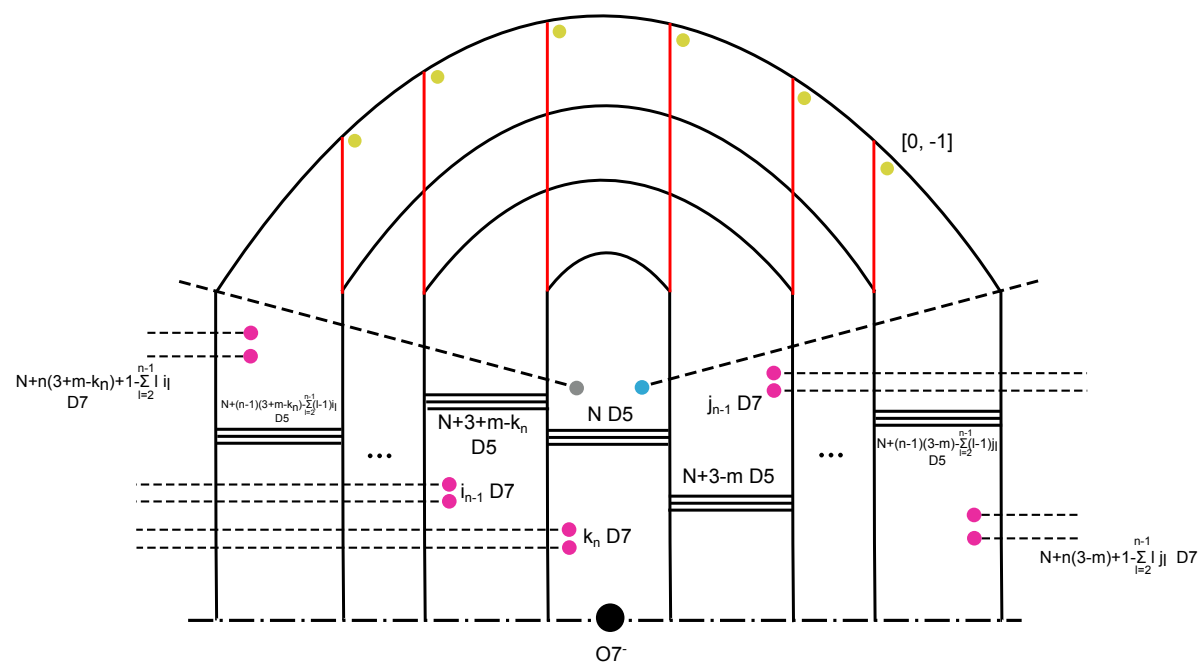

Figure 32. Type IIB brane configuration after the two steps from the one in figure 30 when one $O 7^{-}$-plane is resolved.

$$
\begin{aligned}
& R_{p}=j_{p}, \quad \text { for } \quad 2 \leq p \leq n-1, \\
& R_{1}=N+2 n-n m-\sum_{l=2}^{n-1} l j_{l}+2
\end{aligned}
$$

where $\left(i_{l}+j_{l}\right)=k_{l}$ for $l=1, \cdots, n-1$, and the rank of each gauge group as well as the number of the flavors should be larger than zero at least. When we split only one of the $O 7^{-}$-planes, we obtain the 5-brane configuration in figure 32 after the two steps. After pulling all the 7-branes from figure 32, the final 5-brane web configuration gives rise to a 5 d quiver theory with one $S p$ gauge node and other $S U$ gage nodes with various flavors attached to each node. The explicit expression is

$$
\begin{array}{cccc}
{\left[k_{n}\right]} & {\left[k_{n-1}\right]} & {\left[k_{n-2}\right]} & {\left[k_{1}-2 n+2\right]} \\
\mid & \mid & \mid & \mid \\
\mathrm{Sp}(N+n)-\mathrm{SU}\left(N_{1}\right)-\operatorname{SU}\left(N_{2}\right)-\cdots & \mathrm{SU}\left(N_{n-1}\right),
\end{array}
$$

where

$$
N_{p}=2 N+2 n+4 p-\sum_{l=n-p}^{n-1}(l-n+p+1) k_{l+1}, \quad \text { for } \quad 1 \leq p \leq n-1 .
$$

In this case, the distribution ambiguity of D5-branes and D7-branes does not matter since $i$-th left column from the center and the $i$-th right column from the center are the same column due to the orientifold action.

Since the two types of the theories have the same UV completion as the 6d SCFT, they are dual to each other, which is a further generalization of the claim in section 4.1.3. Furthermore, the 5d $S U$ quiver theory (4.13) has parameters associated to the distribution of D5-branes and D7-branes. Again, all the combinations descend from the same $6 \mathrm{~d}$ theory, we argue that they are dual to each other. The 90 degrees or 45 degrees rotation of the $5 \mathrm{~d}$ theories also give various $5 \mathrm{~d}$ dual theories. 


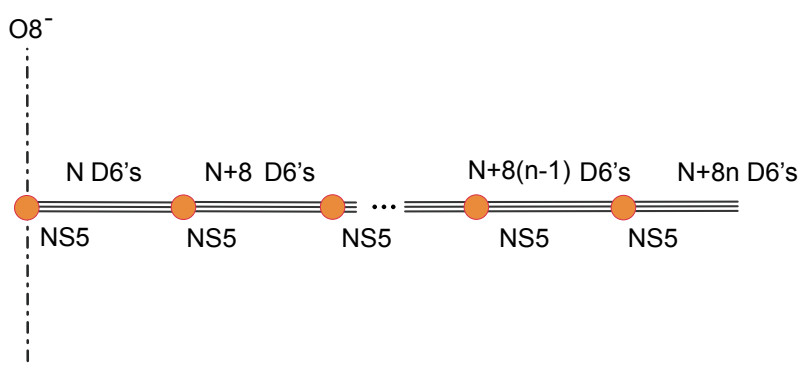

Figure 33. Type IIA brane configuration for the 6d linear quiver theory (4.22).

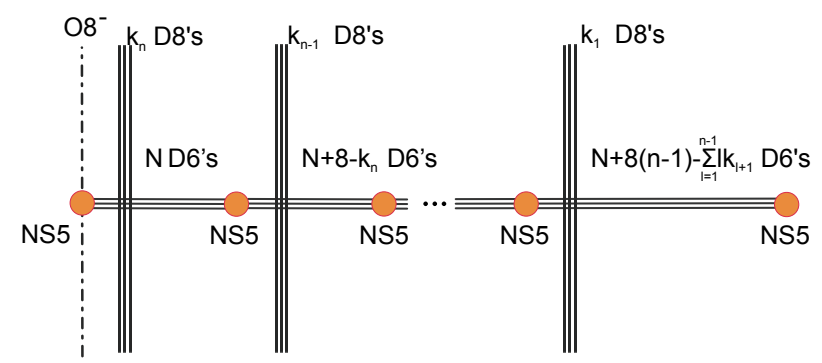

Figure 34. Type IIA brane configuration for the 6d linear quiver theory (4.23).

It is also possible to decouple some flavors from the $5 \mathrm{~d}$ theories. Then, each $5 \mathrm{~d}$ theory will have a5d UV fixed point. Decoupling exactly the same flavors from the dual $5 \mathrm{~d}$ theories should give another dualities between $5 \mathrm{~d}$ theories which has the same UV completion as a $5 \mathrm{~d}$ SCFT.

\subsection{6d SU quivers with an antisymmetric hypermultiplet}

In this subsection, we consider $6 \mathrm{~d} S U$ quiver gauge theories with the gauge node at the edge having one hypermultiplet in the antisymmetric tensor representation, which is a generalization of what we studied in section 3. We consider the generalization analogous to what we did in section 4.1. The simple generalization is the $6 \mathrm{~d}$ linear quiver

$$
6 d[1]_{A}-\mathrm{SU}(N)-\mathrm{SU}(N+8)-\cdots-\mathrm{SU}(N+8(n-1))-[N+8 n],
$$

The type IIA brane configuration is depicted in figure 33. Compared to figure 20, we have an extra NS5-brane on top of $\mathrm{O}^{-}$-plane. The global symmetry is generically $\mathrm{SU}(N+$ $8 n) \times \mathrm{U}(1)$.

We can again further generalize this quiver by Higgsing which is induced exactly by the same mechanism discussed in section 4.1. By considering the Higgsing specified by the Young diagram $[n, \cdots, n, n-1, \cdots, n-1, \cdots, 2, \cdots, 2,1, \cdots, 1]$ where the number of $l$ is $k_{l}$ with a condition $\sum_{l=1}^{n} l k_{l}=2 N+8 n$, we obtain the 6 d quiver gauge theory

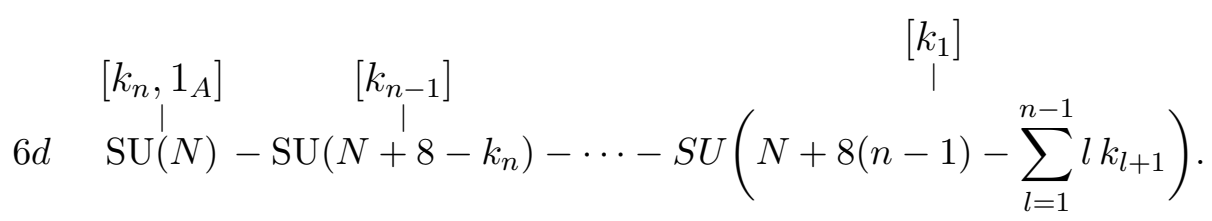




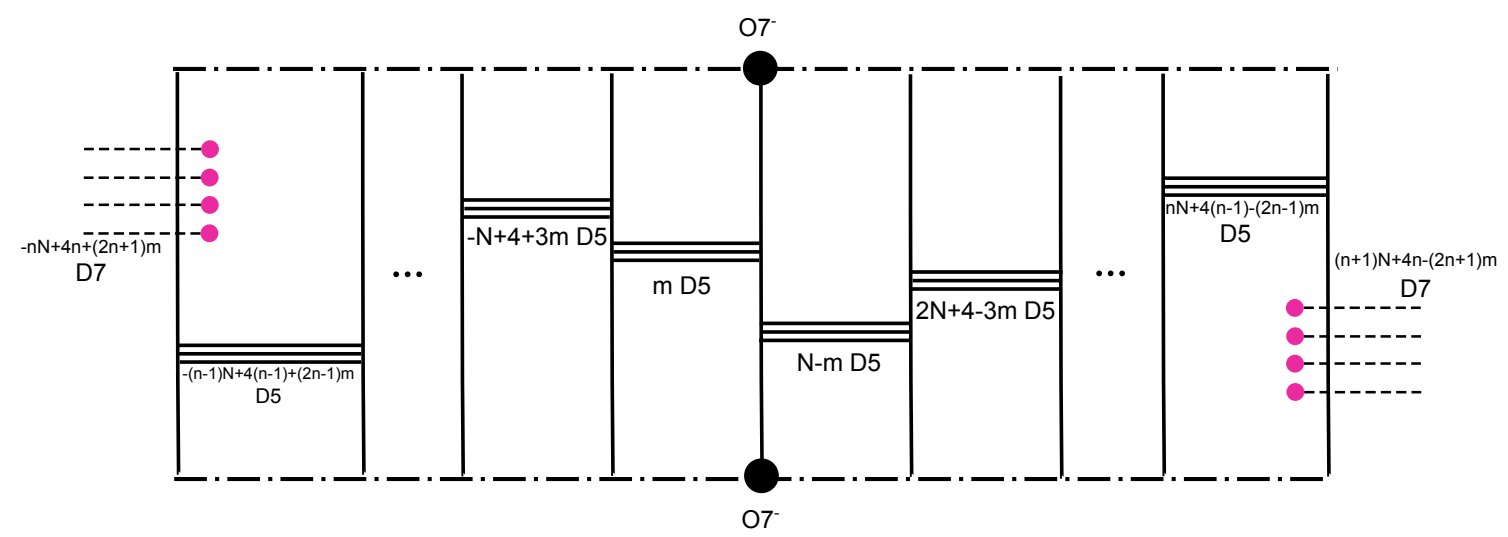

Figure 35. Type IIB brane configuration after performing a T-duality to figure 33 .

The brane setup for this theory is given in figure 34. We will study these $6 \mathrm{~d}$ quivers compactified on $S^{1}$ and consider their 5 d descriptions as well as the 5 d dualities.

\subsubsection{5d SU quivers}

First, we start with the 6 d quiver gauge theory (4.22) on $S^{1}$, T-duality along the $S^{1}$ gives type IIB brane configuration. Analogous to the brane setup discussed in section 4.1.1, the distribution of the D5-branes should be considered. There is no center column in this case and the $N+8(i-1)$ color D5 branes originated from the $i$-th gauge node in 6d quivers (4.22) are distributed into $i$-th left column and $i$-th right column from the center. By imposing the condition that we should obtain a 5 d gauge theory interpretation in the end, it turns out that the distribution ambiguity is again labeled by the single non-negative integer $m$. The resulting distribution is depicted in figure 35 .

Next, we consider the resolution of the two $\mathrm{O}^{-}$-planes attached to 5-branes. The charges of the split 7-branes are determined by the non-negative integer $m$ introduced above due to the condition that one of such 7-branes should be attached to the 5 -brane to which originally $O 7^{-}$-plane was attached. If we fix the splitting type of the upper $O 7^{-}$plane to be $\mathbf{B}, \mathbf{C}$. Then, the splitting type of the lower $O 7^{-}$-plane is determined to be $\mathbf{X}_{[N-2 m-1,1]}, \mathbf{X}_{[N-2 m+1,1]}$. The resolution of the $O 7^{-}$-planes create $n 5$-brane loops as in figure 36. Then, we move the branch cut of these four 7-branes in such a way that some of the D5-branes and D7-branes go across the cut as dictated in the arrow 36. Contrary to the case in figure 25, the number of D5-branes at upper left and lower right is one more than the ones at upper right and lower left. Apart from this small asymmetry, the procedure is quite parallel to section 4.1.1.

After this motion, we obtain the diagram in figure 37. The 5-branes depicted as red lines are the ones coming from the D5-branes which went across the 7-brane monodromy cut. These 5-branes becomes part of the walls splitting each gauge node. Moreover, it turns out that the newly generated 5-brane loop gives extra color D5-branes for each column due to the tuned distribution parametrized by $m$ mentioned previously. Then, from figure 37 , we see that $2 n$ color branes are added to the two columns at the center since all the $n$ 5-branes loops contribute as color D5-branes to these. When we move to the next gauge 


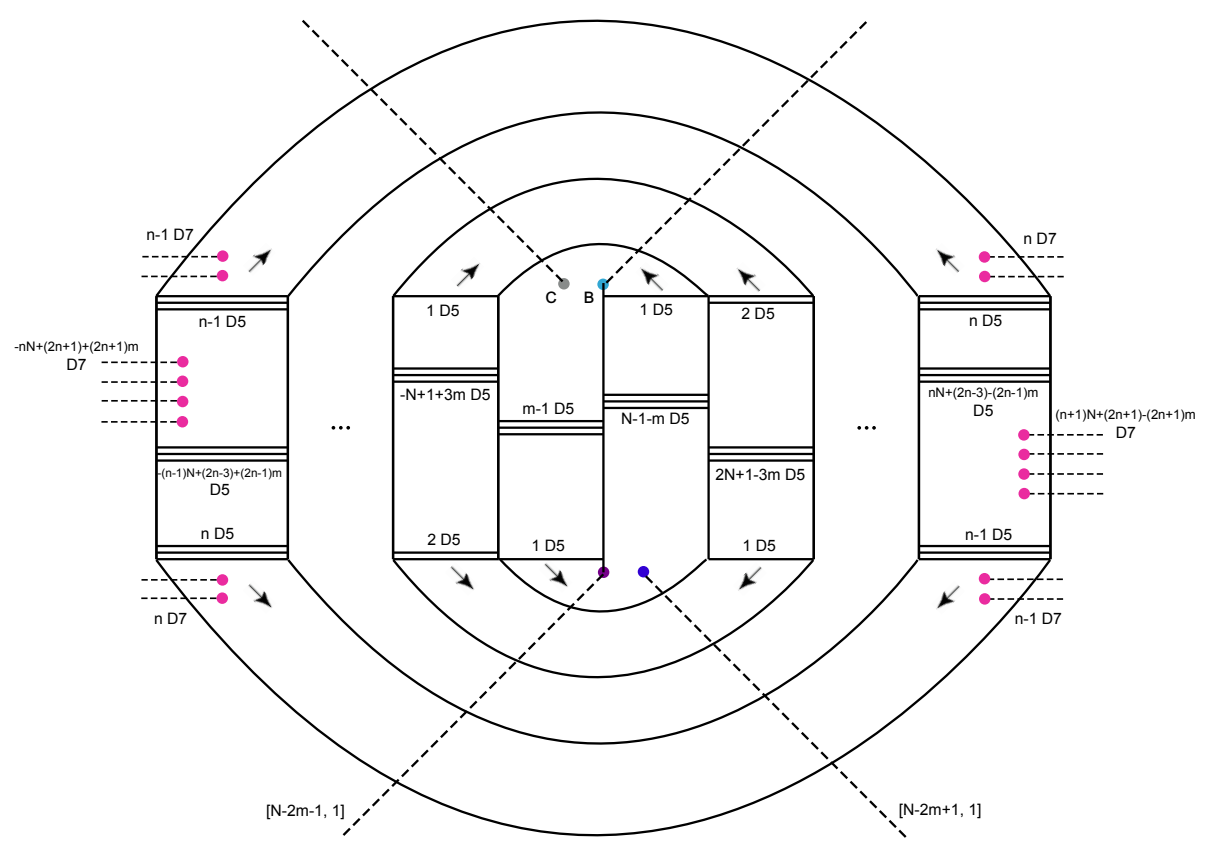

Figure 36. The diagram after resolving two $O 7^{-}$planes in figure 35 . The branch cuts of $\mathbf{B}, \mathbf{C}$ and $\mathbf{X}_{[N+2 m-1,1]} \mathbf{X}_{[N+2 m+1,1]}$ are moved in such a way that the D5-branes and the D7-branes indicated by the arrows go across the cut.

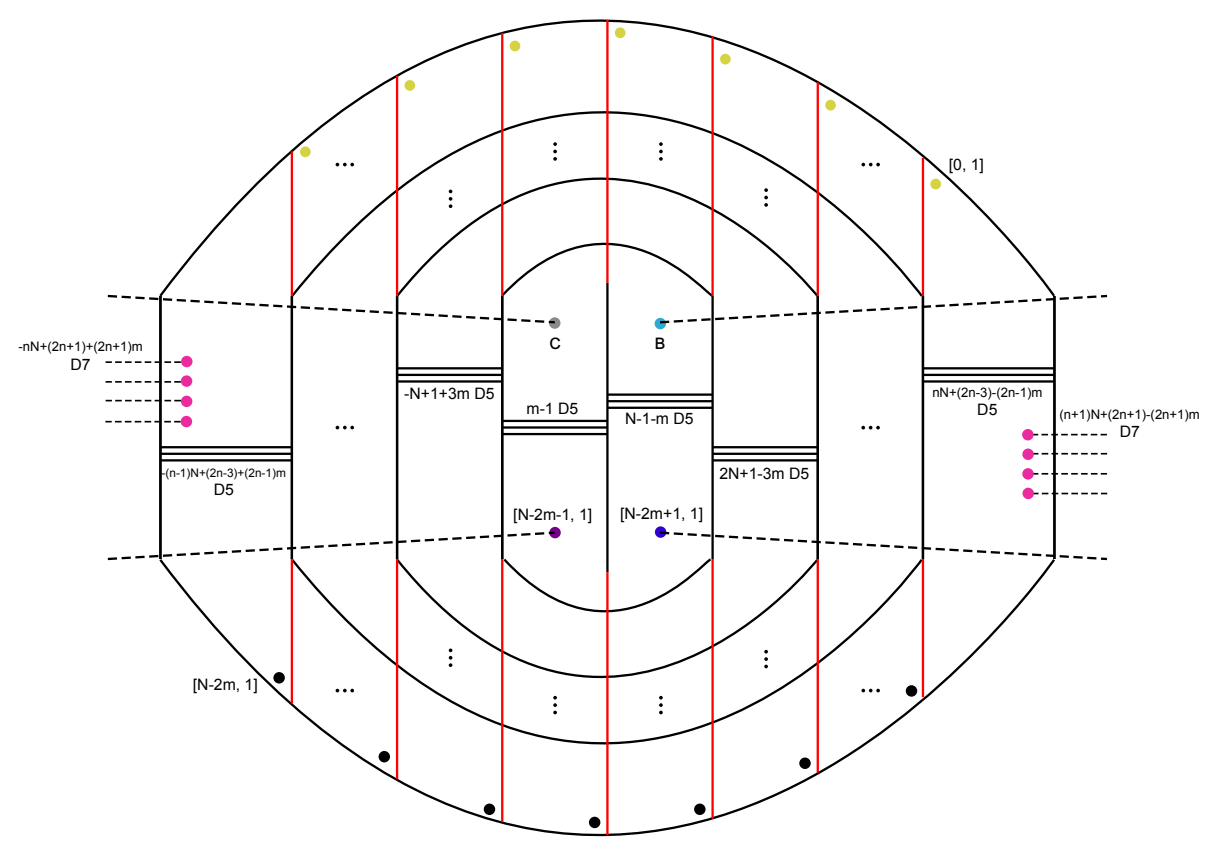

Figure 37. Diagram after the motion in figure 36. 


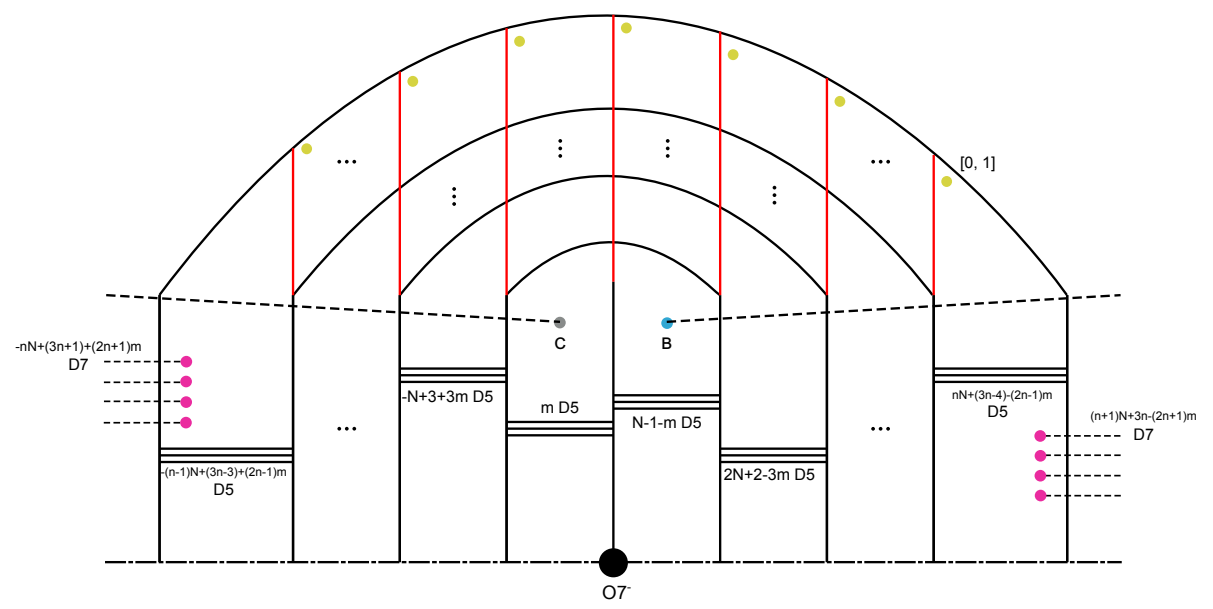

Figure 38. The diagram for the case where only one of the two $O 7^{-}$-planes is resolved.

node, the extra color D5-branes reduce by two and there are only two additional color D5 branes at the columns at the both edges.

Therefore, after moving all the 7-branes outside, we interpret this diagram as the following $5 \mathrm{~d}$ quiver gauge theory:

$$
\left[N_{0}\right]-\mathrm{SU}\left(N_{1}\right)-\mathrm{SU}\left(N_{2}\right)-\cdots-\mathrm{SU}\left(N_{2 n}\right)-\left[N_{2 n+1}\right]
$$

where

$$
\begin{aligned}
N_{0} & =-n N+(2 n+1)(m+1), \\
N_{\ell} & =2 n-1+(-n+\ell) N+(2 n-2 \ell+1) m, \quad \ell=1, \cdots, 2 n, \\
N_{2 n+1} & =(n+1) N-(2 n+1)(m-1) .
\end{aligned}
$$

The parameters are constrained such that the rank of each gauge group or the number of the flavors should be positive. In summary, we see that the 6 d quiver theory (4.22) on $S^{1}$ is described by the $5 \mathrm{~d}$ quiver gauge theory (4.24).

\subsubsection{5d SU quivers with an antisymmetric hypermultiplet}

Here, we consider the case of resolving only one of the two $\mathrm{O}^{-}$-planes in the diagram in figure 35. After we resolve the upper $\mathrm{O}^{-}$-plane into $\mathbf{B}$ and $\mathbf{C} 7$-branes, we consider the same procedure, namely we move the branch cuts of the $\mathbf{B}$ and $\mathbf{C} 7$-branes so that some of the D7-branes and D5-branes cross them. The explicit motion of the branch cuts or equivalently the motion of D7-branes and D5-branes is exactly the same one that we performed for the upper part of the diagram in figure 36, while we keep the lower half part as it is. After moving the branch cuts, it is straightforward to see that we obtain the diagram depicted in figure 38. In this case, the distribution ambiguity does not matter as the $i$-th left column from the center is actually the same column as the $i$-th right column from the center by the orientifold action. In other words, we have $n S U$ gauge nodes. 
After moving all the 7-branes outside, we obtain a 5-brane web diagram. It is possible to can read off from the diagram that this theory is the following $5 \mathrm{~d}$ quiver gauge theory:

$$
[1]_{A}-\mathrm{SU}(N+2 n-1)-\mathrm{SU}(N+2 n+3)-\cdots-\mathrm{SU}(N+6 n-5)-[N+6 n+1] .
$$

Note that we have an anti-symmetric hypermultiplet at the left end node as a 5-brane is attached to the lower unresolved $O 7^{-}$-plane [39].

\subsubsection{5d dualities}

In section 4.2.1 and in section 4.2.2, we have discussed two different types of $5 \mathrm{~d}$ description for the $6 \mathrm{~d}$ theory (4.22), which are $5 \mathrm{~d}$ quivers (4.24) and (4.26). Since their type IIB diagrams come from the identical type IIA diagram for the $6 \mathrm{~d}$ theory, we claim that these two types of $5 \mathrm{~d}$ theory are dual to each other, which means that they have the identical 6d UV fixed point.

When we combine mass deformation and S-duality as discussed in section 3.6, we will be able to obtain various dual $5 \mathrm{~d}$ theories. Furthermore, by considering the flavor decoupling limit, all these dualities can be also reduced to the dualities for another set of theories which have an identical 5d UV fixed point. Classifying all these dual 5d theories would be interesting future problem.

\subsubsection{Higgsed cases}

Now, we go on to the $6 \mathrm{~d}$ theory (4.23), which is obtained by Higgsing (4.22). Diagrammatic derivation of the $5 \mathrm{~d}$ description is quite parallel to what we did in section 4.2 .1 and in section 4.2.2. The different point is that some of flavor D7-branes exist at internal columns. The effect of such D7-branes are again quite parallel to what we discussed in section 4.1.4. Here, we summarize the resulting $5 \mathrm{~d}$ description.

When we resolve two $\mathrm{O}^{-}$planes, we obtain the $5 \mathrm{~d}$ quivers

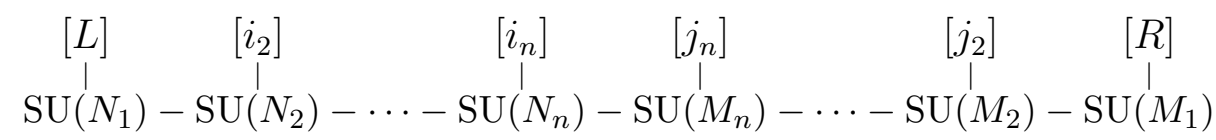

where

$$
\begin{aligned}
L & =-n N+(2 n+1)(m+1)-\sum_{l=2}^{n} l i_{l}, \\
N_{p} & =2 n-1+(-n+p) N+(2 n-2 p+1) m-\sum_{l=p+1}^{n}(l-p) i_{l}, \quad p=1, \cdots, n, \\
M_{p} & =2 n-1+(n-p+1) N-(2 n-2 p+1) m-\sum_{l=p+1}^{n}(l-p) j_{l}, \quad p=1, \cdots, n, \\
R & =(n+1) N-(2 n+1)(m-1)-\sum_{l=2}^{n} l j_{l} .
\end{aligned}
$$

where $i_{l}+j_{l}=k_{l}$. 


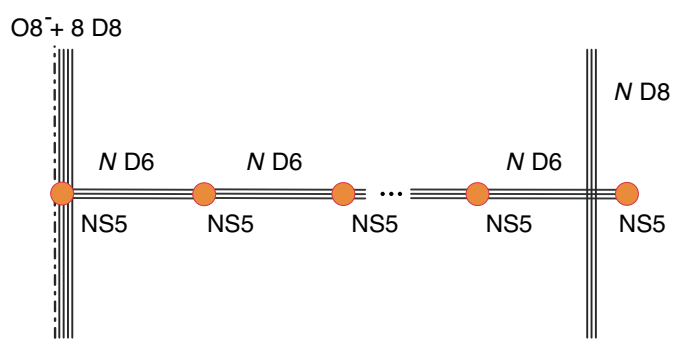

Figure 39. $6 \mathrm{~d}$ brane configuration for the quiver $\left[1_{A}, 8\right]-\mathrm{SU}(N)-\mathrm{SU}(N)-\cdots-\mathrm{SU}(N)-[N]$ which has an antisymmetric hypermultiplet and eight fundamental flavors of the leftmost gauge group of the quiver also coupled to tensor multiplets.

When we resolve only one out of the two $\mathrm{O}^{-}$planes, we obtain the 5 d quivers

$$
\begin{array}{ccccc}
{\left[k_{n}, 1_{A}\right]} & {\left[k_{n-1}\right]} & {\left[k_{n-2}\right]} & {\left[k_{2}\right]} & {\left[k_{1}-2 n+1\right]} \\
\mid & \mid & \mid & \mid & \mid \\
\mathrm{SU}\left(N_{1}\right) & -\mathrm{SU}\left(N_{2}\right)-\mathrm{SU}\left(N_{3}\right)-\cdots-\mathrm{SU}\left(N_{n-1}\right)- & \mathrm{SU}\left(N_{n}\right)
\end{array}
$$

where

$$
N_{s}=N+2 n-5+4 s-\sum_{l=1}^{s-1} l k_{l+1+n-s} .
$$

We claim that these two types of the theories have the same 6d UV fixed point. Also, the $5 \mathrm{~d}$ SU quiver theory (4.27) with any possible value for $m, i_{l}$, and $j_{l}$ give the set of dual theories. S-duality of these $5 \mathrm{~d}$ theories will also give various dual theories. Flavor decoupling limit will give other set of dual theories which have the same 5d UV fixed point.

\section{Special cases: the $6 \mathrm{~d}$ quiver gauge theories of $\mathrm{Or}^{-}+8 \mathrm{D} 8^{-}$'s configura- tion}

In this section, we consider the 6d SCFT configurations in tensor branch which are composed of an $\mathrm{O}^{-}$plane together with eight D8 branes, leading to indefinite sequence of quiver type made of D6 branes stretched between two NS5 branes. These cases can be, in principle, obtained through the Higgsing of general $6 \mathrm{~d}$ brane configuration discussed in the previous section. It is however non-trivial to get $5 \mathrm{~d}$ description following the procedure described in the previous sections. It is partial because, to have Lagrangian description in 5d, many D7 branes are converted to other 7-branes resulting in lack of flavor 7-branes. On the other hand, we discuss below that one can still have an Lagrangian description in the S-dual frame or by implementing the $\mathrm{SL}(2, \mathbb{Z})$ transformation on the $(p, q)$ plane. These special cases also give intriguing dual pictures depending the resolution of the $O 7^{-}$plane into a pair of 7-brane. 


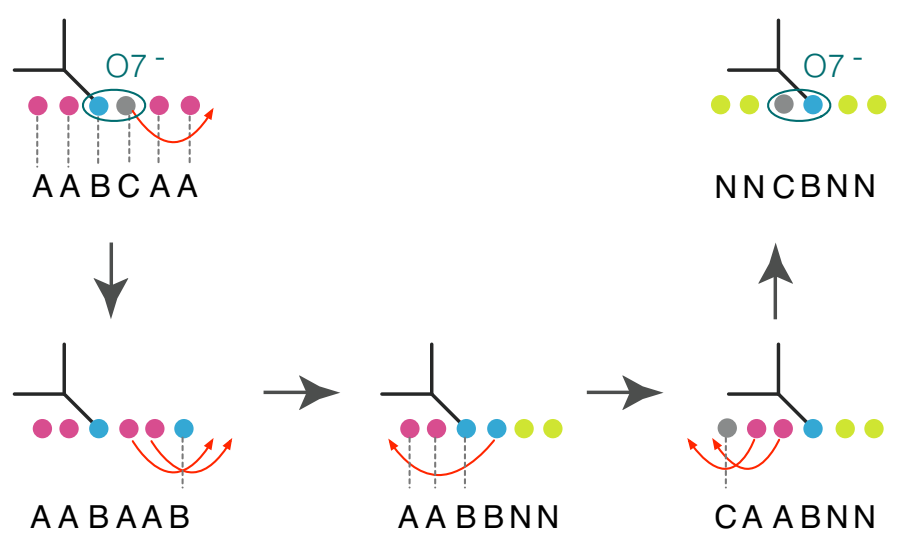

Figure 40. (i) $\mathrm{An} \mathrm{SL}(2, \mathbb{Z})$ invariant 7-brane combination where $\mathbf{B}$ 7-brane is attached to 5-brane. A sequence of monodromy analysis makes $\mathbf{A}^{\mathbf{2}} \mathbf{B} \mathbf{C A}^{2}$ into $\mathbf{N}^{2} \mathbf{C B N}^{2}$ which will be converted into $\mathbf{A}^{\mathbf{2}} \mathbf{B C A}^{2}$ again via an S-dual action.

\section{$5.16 \mathrm{~d}\left[1_{A}, 8\right]-\mathrm{SU}(N)-\mathrm{SU}(N)-\cdots-[N]$ quiver}

We first consider the case where an $\mathrm{O}^{-}$with eight D8 branes and a NS5 brane being on top of the $\mathrm{O}^{-}$plane,

$$
6 \mathrm{~d}\left[1_{A}, 8\right]-\underbrace{\mathrm{SU}(N)-\mathrm{SU}(N)-\cdots-\mathrm{SU}(N)}_{n \text { nodes }}-[N] .
$$

The corresponding $6 \mathrm{~d}$ brane configuration is given in figure 39 .

$\mathrm{SL}(2, \mathbb{Z})$ invariant 7 -brane combinations. We will discuss various dualities along the reduction of the $6 \mathrm{~d}$ theories to $5 \mathrm{~d}$ theories by analyzing the 7 -brane monodromies with or without the $O 7^{-}$planes. In order to see the dual picture, it is useful to consider combinations of 7 -branes which are invariant under the $\operatorname{SL}(2, \mathbb{Z})$ transformation on the $(p, q)$ 5-brane web plane. An $07^{-}$plane and four D7 branes would be an obvious example among many $\mathrm{SL}(2, \mathbb{Z})$ invariant 7-brane combinations, as the total monodromy of them is proportional to minus identity matrix. It follows immediately that a pair of 7 -branes as a resolution of the $O 7^{-}$plane, together with four $\mathrm{D} 7$ branes, is thus $\operatorname{SL}(2, \mathbb{Z})$ invariant. For instance, $(\mathbf{B}, \mathbf{C})$ or $(\mathbf{N}, \mathbf{X}) 7$-branes with four D7 branes (four A $\mathbf{A}$-branes). We will show a few distinctive examples of such combinations involving 7-branes from different resolutions of the $O 7^{-}$plane. In particular, the cases where one of the 7-branes is attached to a 5brane of the same charge will give rise to fruitful dualities among the resulting $5 \mathrm{~d}$ quiver gauge theories. We consider such combinations frequently appearing through T-duality when reducing a $6 \mathrm{~d}$ theory to $5 \mathrm{~d}$.

As discussed earlier, when going from $6 \mathrm{~d}$ to $5 \mathrm{~d}$, one can distribute D7 branes and D5 branes with a suitable Wilson line, which enables us to allocate any number of D7 branes close to $\mathrm{O}^{-}$planes. Regarding resolution of $\mathrm{O}^{-}$planes, we restrict ourselves to two distinctive resolutions of $O 7^{-}$planes, which is either $(\mathbf{B}, \mathbf{C})$ or $\left(\mathbf{N}, \mathbf{X}_{[\mathbf{2}, \mathbf{1}]}\right)$ 7-brane pair.

(i). Consider the 7-brane configuration $\mathbf{A}^{\mathbf{2}} \mathbf{B C A} \mathbf{A}^{2}$ where $\mathbf{B}$ is attached to a $(1,-1)$ 5-brane as depicted in figure 40. It is straightforward to show that it can be expressed 


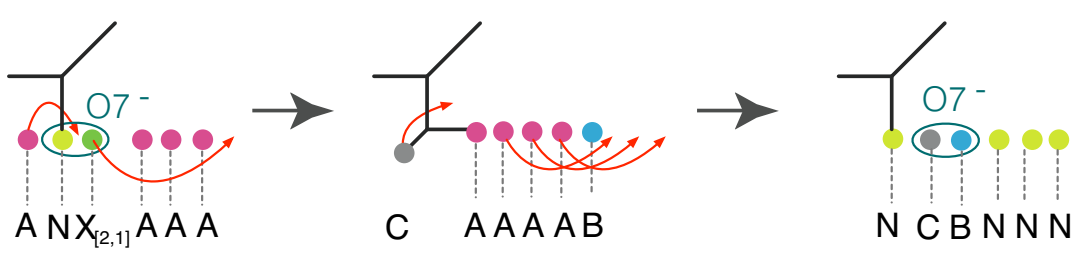

Figure 41. (ii) An $\operatorname{SL}(2, \mathbb{Z})$ invariant 7-brane combination where the 7-brane $\mathbf{N}$ is attached to $(0,1)$ 5-brane. A sequence of monodromy analysis makes $\mathbf{A N} \mathbf{X}_{[\mathbf{2}, \mathbf{1}]} \mathbf{A}^{\mathbf{3}}$ into $\mathbf{N C B N}^{\mathbf{3}}$.

as the $\mathbf{N}^{2} \mathbf{C B N}^{2}$ 7-brane configuration where $\mathbf{B}$ is still attached to the 5 -brane ${ }^{8}$ (See figure 40). By performing an S-duality transformation $(p, q) \rightarrow(-q, p)$, one sees that $\mathbf{A}^{\mathbf{2}} \mathbf{B C A}^{\mathbf{2}}$ is an S-dual invariant combination

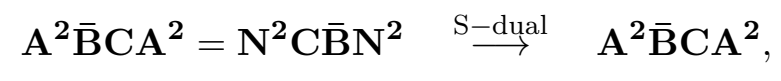

where we used a bar " - " to denote the 7-brane is attached to a 5-brane of the same charge.

(ii). Another example is $\mathbf{A N X} \mathbf{X}_{[\mathbf{2}, \mathbf{1}]} \mathbf{A}^{\mathbf{3}}$ with $\mathbf{N}$ 7-brane being attached to $(0,1)$ 5-brane. See figure 41. Again a little monodromy calculation ${ }^{9}$ leads that $\mathbf{A N} \mathbf{X}_{[\mathbf{2 , 1}]} \mathbf{A}^{\mathbf{3}}$ is an $\mathrm{SL}(2, \mathbb{Z})$ invariant 7-brane combination

$$
\mathbf{A} \overline{\mathbf{N}} \mathbf{X}_{[2,1]} \mathbf{A}^{3}=\overline{\mathbf{N}} \mathrm{CBN}^{3} \stackrel{\text { S-dual }}{\longrightarrow} \overline{\mathrm{ABCA}^{3}}
$$

as $\mathbf{N} \mathbf{X}_{[\mathbf{2 , 1}]}$ and $\mathbf{B C}$ are related by a successive application $T$-action of the $\mathrm{SL}(2, \mathbb{Z})$ transformation. Notice that before taking an S-duality, the $\mathbf{N} 7$-brane is attached to $(0,1) 5$-brane, but after the S-duality and a manipulation of monodromies as well as the Hanany-Witten transition, the A 7-brane is attached to a D5 brane. This procedure plays an important role in showing a dual description of 5 d quiver theories which we discuss below.

(iii). When no 5-brane is attached to 7 -branes, it is easier for one to see $\operatorname{SL}(2, \mathbb{Z})$ invariance. For instance, consider $\mathbf{A}^{\mathbf{2}} \mathbf{O} \mathbf{7}^{-} \mathbf{A}^{\mathbf{2}}$ or $\mathbf{A}^{\mathbf{2}} \mathbf{B C} \mathbf{A}^{\mathbf{2}}$. (See also figure 42.) Using the monodromy, one finds that $\mathbf{A}^{\mathbf{2}} \mathbf{B C} \mathbf{A}^{\mathbf{2}}$ is S-dual invariant

$$
\mathrm{A}^{2} \mathrm{BCA}^{2}=\mathrm{CA}^{2} \mathbf{A}^{2} \mathrm{~B}=\mathrm{N}^{2} \mathrm{CBN}^{2} \stackrel{\mathrm{S}-\text { dual }}{\longrightarrow} \mathrm{A}^{2} \mathbf{B C A}^{2} .
$$

${ }^{8}$ Using the 7-brane monodromies, one finds that

$$
\mathbf{C A}=\mathbf{A N}=\mathbf{N C}, \quad \mathbf{N A}=\mathbf{A B}=\mathbf{B N}, \quad \text { or } \quad \mathbf{N}^{2} \mathbf{C}=\mathbf{C A}^{2}=\mathbf{A}^{2} \mathbf{B}=\mathbf{B N}^{2},
$$

which yield

$$
\mathrm{A}^{2} \overline{\mathrm{BCA}}{ }^{2}=\mathrm{A}^{2} \overline{\mathrm{B}} \mathrm{A}^{2} \mathrm{~B}=\mathrm{A}^{2} \overline{\mathrm{B}} \mathrm{BN}{ }^{2}=\mathrm{CA}^{2} \overline{\mathrm{B}} \mathrm{N}^{2}=\mathrm{N}^{2} \mathrm{C}_{\overline{\mathrm{B}}}{ }^{2}
$$

where the 7-brane $\overline{\mathbf{B}}$ is attached to $(1,-1)$ 5-brane. A pictorial version of this monodromies is given in figure 40 .

${ }^{9}$ The relevant monodromy relation for this case is

$$
\mathbf{X}_{[2,1]} \mathbf{A}=\mathbf{A C}, \quad \mathbf{C A}=\mathbf{A N}=\mathbf{N C},
$$

yielding

$$
\operatorname{ANX}_{[2,1]} A^{3}=\mathrm{CA}^{3} \mathrm{~B}=\mathrm{NCBN}^{3}
$$




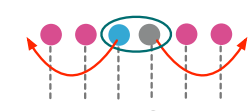

A A B C A A

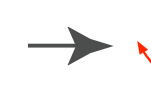

C A A A A B

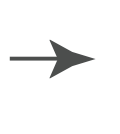

\section{NNCBNN}

Figure 42. (iii) An SL(2, $\mathbb{Z})$ invariant 7-brane combination where no 7-brane is attached to 5-brane. A sequence of monodromy analysis makes $\mathbf{A}^{2} \mathbf{B} \mathbf{C A}^{2}$ into $\mathbf{N}^{2} \mathbf{C B N}^{2}$.
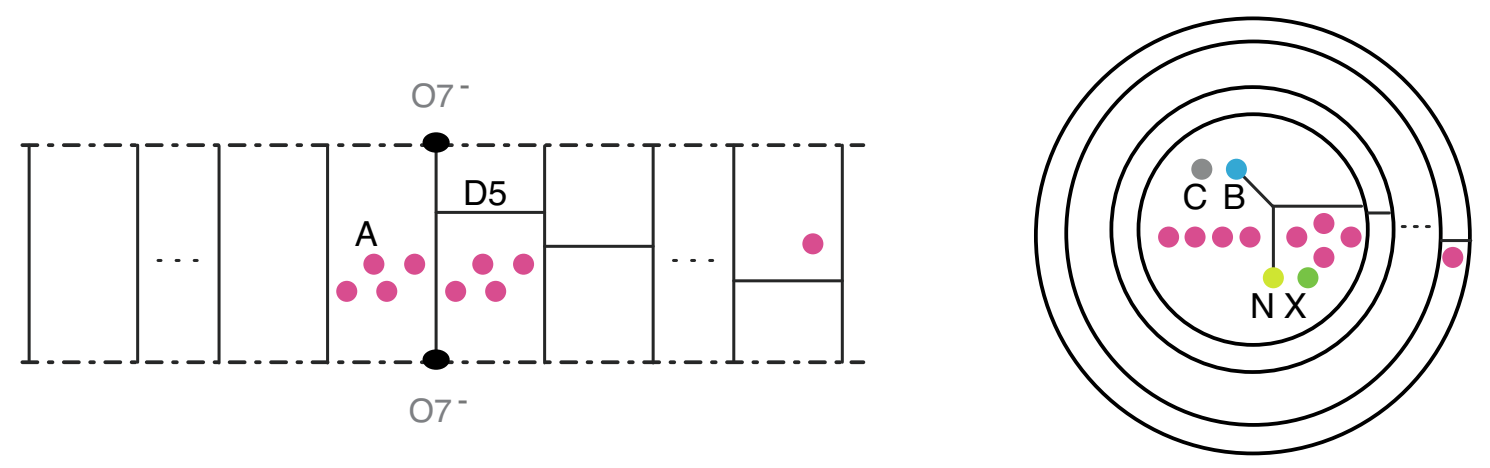

Figure 43. Left: $5 \mathrm{~d}$ brane configuration for the quiver $\left[1_{A}, 8\right]-\mathrm{SU}(1)-\mathrm{SU}(1)-\cdots-\mathrm{SU}(1)-[1]$. Right: splitting the $\mathrm{O}^{-}$plane gives 5 -brane loops which are denoted as circular loops.

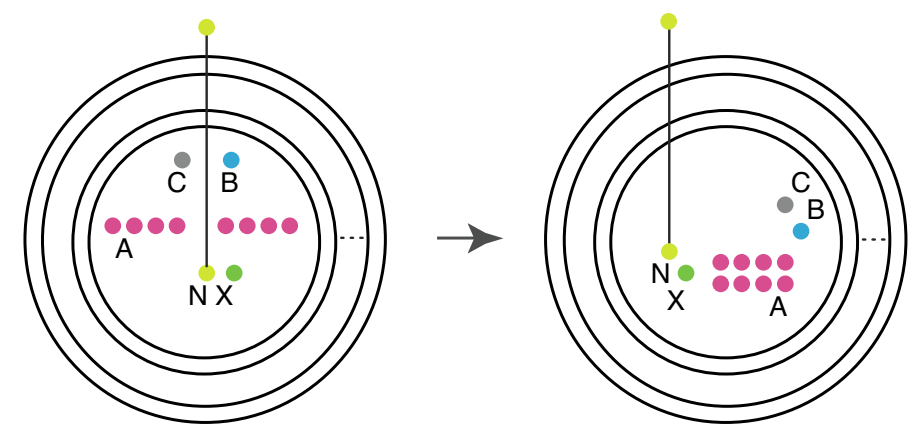

Figure 44. $6 \mathrm{~d}\left[1_{A}, 8\right]-\mathrm{SU}(1)-\mathrm{SU}(1)-\cdots-\mathrm{SU}(1)-[1]$ giving $5 \mathrm{~d} \operatorname{Sp}(n)$ with $N_{f}=8$ and $N_{a}=1$. From the figure on the right of figure 43, one moves $\mathbf{B} 7$-brane along the direction of the charge $(1,-1)$ so that the $\mathbf{B}$ brane comes a freely floating 7 -brane. This procedure gives a configuration on the left here. Using the 7-brane monodromy, one can put all the 7-brane on one side like the figure on the right.

With all these $\mathrm{SL}(2, \mathbb{Z})$ invariant 7 -brane combinations, (i), (ii), and (iii), we study special brane configurations which yield various $5 \mathrm{~d}$ quiver theories.

$6 \mathrm{~d}\left[1_{A}, 8\right]-\mathrm{SU}(1)-\mathrm{SU}(1)-\cdots-[1]$ quiver and 5d Sp theory with $N_{f}=8$ and $\boldsymbol{N}_{\boldsymbol{a}}=\mathbf{1}$. For the $N=1$ case with $n$ quiver nodes, we claim that the $6 \mathrm{~d}$ brane configuration gives rise to $5 \mathrm{~d} \operatorname{Sp}(n)$ gauge theory with $N_{f}=8$ and $N_{a}=1$ hypermultiplets:

$6 \mathrm{~d}\left[1_{A}, 8\right]-\underbrace{\mathrm{SU}(1)-\cdots-\mathrm{SU}(1)}_{n \text { nodes }}-[1] \Rightarrow 5 \mathrm{~d} \operatorname{Sp}(n)$ theory with $N_{f}=8 \& N_{a}=1$ 
A circle compactification followed by T-duality makes an $O 8^{-}$into two $O 7^{-}$'s separated maximally along the T-dual circle. NS5 brane stuck on the $08^{-}$is now a brane junction of NS5 brane connecting two $O 7^{-}$s as well as a D5 brane. Because of this junction, when resolving the $\mathrm{O7}^{-}$planes into two pairs of two 7-branes, each $\mathrm{O7}^{-}$is resolved differently, for instance, $([-1,1]$ and $[1,1]) 7$-branes for an $O 7^{-}$plane, and $([0,1]$ and $[2,1]) 7$-branes for the other. See figure 43. From 7-brane monodromies for this configuration, one finds that it leads to the configuration for $5 \mathrm{~d} \operatorname{Sp}(n)$ gauge theory with $N_{f}=8$ and $N_{a}=1$ flavors, which describes a circle compactification of $6 \mathrm{~d}$ higher rank E-string theory. We note that the web diagram for higher rank E-string theory of massless antisymmetric hypermultiplet was already discussed in [30] which is made out of eight D7 branes, and its flavor decoupling limit was also discussed in [41] as a limit taking masses of D7 branes to infinite. Notice however that we now have nine D7 branes in the original setup, and as a consequence of 7 brane monodromy, one of $\mathrm{D} 7$ branes is now converted to a $[0,1]$-brane. Hence the remaining D7 branes account eight flavors, but as we have the $[0,1] 7$-brane which does not exit for the web configuration for the massless antisymmetric hypermultiplet, this 7-brane is thus associated with a non-zero mass of antisymmetric hypermultiplet. See figure 44. Taking the flavor decoupling limit for the fundamental flavors, one would find $5 \mathrm{~d}$ web configuration for $\operatorname{Sp}(n)$ gauge theory with $N_{f} \leq 7$ and $N_{a}=1$ flavors, given in [39]. By pulling out all the 7-branes, it is not so difficult for one to find that it makes a Tao web diagram.

$N=2$ and $n$ nodes: duality between $5 d \operatorname{SU}(2 n+1)$ with $N_{a}=2$ and $\operatorname{Sp}(n) \times$ $\mathbf{S p}(\boldsymbol{n})$. Using an aforementioned $\operatorname{SL}(2, \mathbb{Z})$ invariant combination with $(\mathbf{B}, \mathbf{C})$ 7-branes (a resolution of the $O 7^{-}$plane) and four D7 brane, one finds that the $N=2$ case of $n$ quiver nodes

$$
6 \mathrm{~d}\left[1_{A}, 8\right]-\underbrace{\mathrm{SU}(2)-\cdots-\mathrm{SU}(2)}_{n \text { nodes }}-[2]
$$

gives rise to two $5 \mathrm{~d}$ theories. Firstly, reducing it to $5 \mathrm{~d}$, we can take the S-duality in a $\mathrm{SL}(2, \mathbb{Z})$ invariant way that the 7 -branes are rearranging themselves to respect the original 7-brane structure. As this case can be understood as the case when $N$ is even, which we will discuss in what follows. We state that the resulting $5 \mathrm{~d}$ theories. This case leads to $5 \mathrm{~d}$ $\mathrm{SU}(2 n+1)$ gauge theory with two antisymmetric hypermultiplets

$$
5 \mathrm{~d}\left[1_{A}, 4\right]-\mathrm{SU}(2 n+1)-\left[1_{A}, 4\right] .
$$

On the other hand, a different resolution of the $O 7^{-}$plane, e.g., $O 7^{-} \rightarrow \mathbf{N}, \mathbf{X}_{[\mathbf{2}, \mathbf{1}]}$, yields the quiver of $\operatorname{Sp}(n) \times \operatorname{Sp}(n)$ theory only with fundamental hypermultiplets

$$
5 \mathrm{~d}[4]-\operatorname{Sp}(n)-\operatorname{Sp}(n)-[4] \text {. }
$$

This suggests that these two $5 \mathrm{~d}$ theories are dual to each other in the sense that they have the same UV fixed points.

$\boldsymbol{N}=$ even: duality between Sp quiver and SU quiver. For $N=2 m$ and $n$ quiver nodes, $6 \mathrm{~d}$ theory is

$$
6 \mathrm{~d}\left[1_{A}, 8\right]-\underbrace{\mathrm{SU}(2 m)-\cdots-\mathrm{SU}(2 m)}_{n \text { nodes }}-[2 m] .
$$




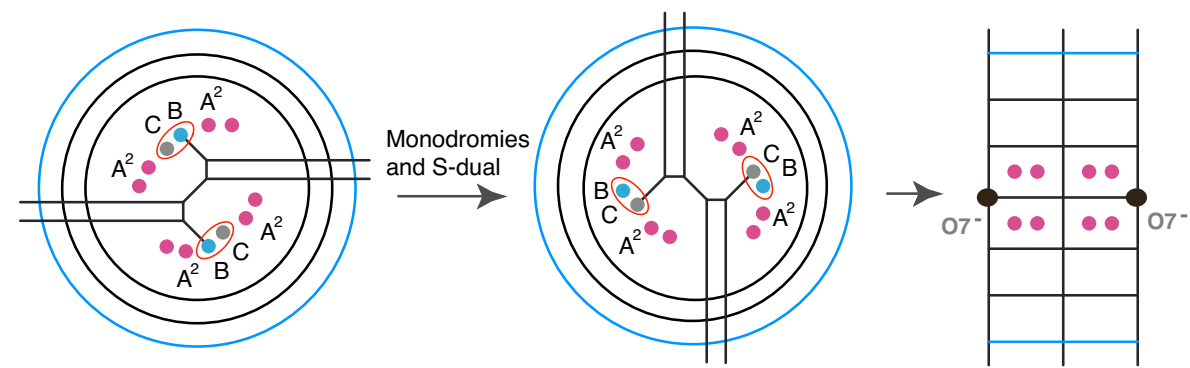

Figure 45. A T-dual version of $6 \mathrm{~d}\left[1_{A}, 8\right]-\mathrm{SU}(4)-\mathrm{SU}(4)-\mathrm{SU}(4)-[4]$ yielding $5 \mathrm{~d}\left[1_{A}, 4\right]-$ $\mathrm{SU}(7)-\mathrm{SU}(7)-\left[1_{A}, 4\right]$ implementing S-dual invariant 7-brane combinations.

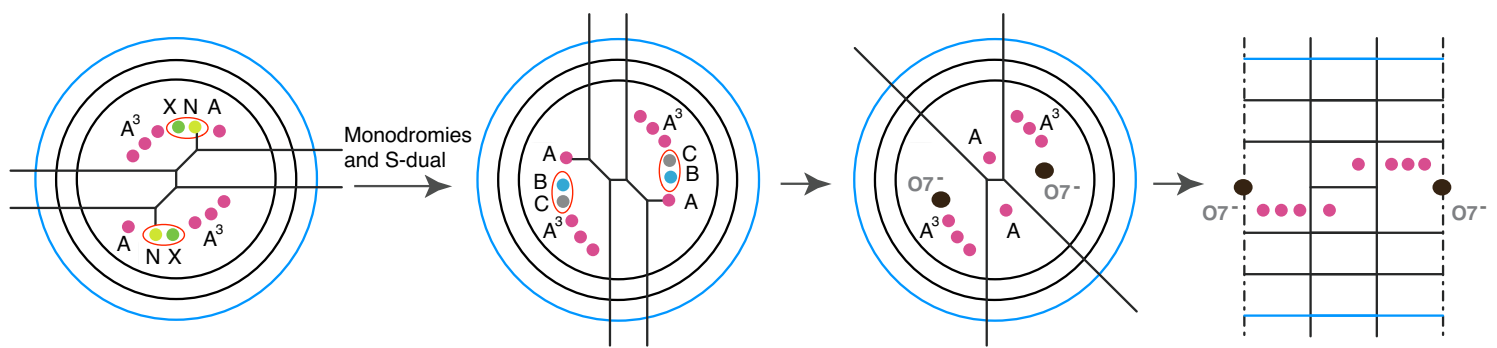

Figure 46. A T-dual version of $6 \mathrm{~d}\left[1_{A}, 8\right]-\mathrm{SU}(4)-\mathrm{SU}(4)-\mathrm{SU}(4)-[4]$ yielding $5 \mathrm{~d}[3]-\mathrm{Sp}(3)-$ $(\mathrm{SU}(7)-[2])-\mathrm{Sp}(3)-[3]$ implementing S-dual invariant 7-brane combinations.

Again, one uses $\mathrm{SL}(2, \mathbb{Z})$ invariant 7-brane combinations introduced earlier and S-duality to obtain the following $5 \mathrm{~d}$ theories:

$$
\begin{aligned}
& 5 \mathrm{~d}\left[1_{A}, 4\right]-\underbrace{\mathrm{SU}(2 n+1)-\mathrm{SU}(2 n+1)-\cdots-\mathrm{SU}(2 n+1)}_{m \text { nodes }}-\left[1_{A}, 4\right] \\
& 5 \mathrm{~d}[3]-\underbrace{\mathrm{Sp}(n)-\mathrm{SU}(2 n+1)-\mathrm{SU}(2 n+1)-\cdots-\mathrm{SU}(2 n+1)-\mathrm{SU}(2 n+1)-\mathrm{Sp}(n)}_{m+1 \text { nodes }}-[3] .
\end{aligned}
$$

The first case is realized when we split the $O 7^{-}$into $\mathbf{B}$ and $\mathbf{C} 7$-branes. The second case is realized when we split the $O 7^{-}$into $\mathbf{N}$ and $\mathbf{X}_{[\mathbf{2}, \mathbf{1}]} 7$-branes.

As a representative example, the brane configuration for $6 \mathrm{~d}\left[1_{A}, 8\right]-\mathrm{SU}(4)-\mathrm{SU}(4)-$ $\mathrm{SU}(4)-[4](m=2$ and $n=3)$ yielding

$$
5 \mathrm{~d}\left[1_{A}, 4\right]-\mathrm{SU}(7)-\mathrm{SU}(7)-\left[1_{A}, 4\right]
$$

is depicted in figure 45. As there are eight D7 branes, one can relocate and arrange D7 branes with suitable Wilson lines, such that four D7 branes are located close to each the $O 7^{-}$plane so to make an $\mathrm{SL}(2, \mathbb{Z})$ invariant 7 -brane combination including the resolution of the $\mathrm{O}^{-}$plane into the $\mathbf{B}, \mathbf{C}$ 7-branes. Performing an S-duality and also taking a weak coupling limit to obtain the $\mathrm{O}^{-}$plane out of two suitable 7 -branes, one finds that the 


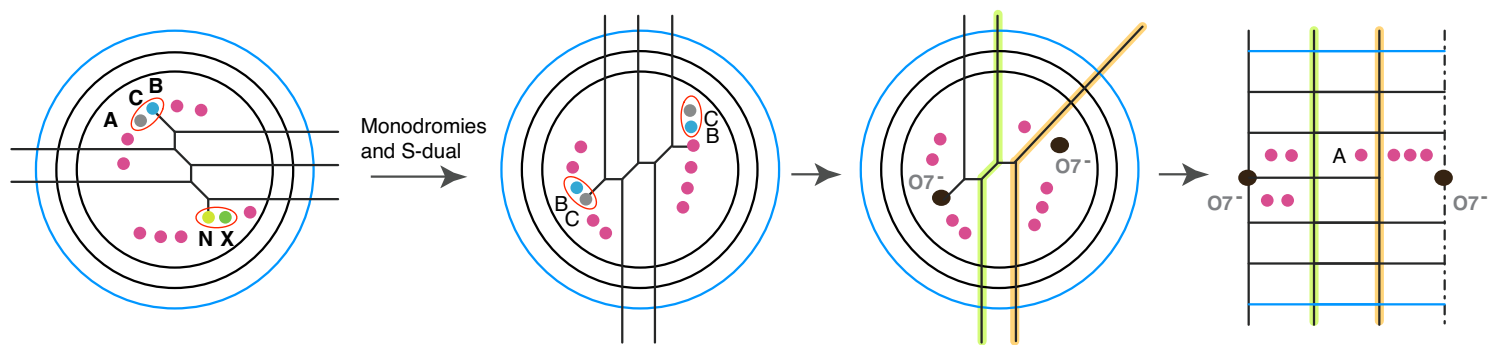

Figure 47. A T-dual version of $6 \mathrm{~d}\left[1_{A}, 8\right]-\mathrm{SU}(5)-\mathrm{SU}(5)-\mathrm{SU}(5)-[5]$ yielding $5 \mathrm{~d}\left[1_{A}, 4\right]-$ $\mathrm{SU}(7)-(\mathrm{SU}(7)-[1])-\mathrm{Sp}(3)-[3]$ implementing S-dual invariant 7-brane combinations.

resulting web configuration gives rise to the quiver gauge theory with an antisymmetric hypermultiplet at the edge gauge node. In a different way of resolving the $O 7^{-}$planes, $6 \mathrm{~d}$ brane configuration yields a seemingly different $5 \mathrm{~d}$ quiver theory

$$
\begin{gathered}
{[2]} \\
5 \mathrm{~d}[3]-\mathrm{Sp}(3)-\mathrm{SU}(7)-\mathrm{Sp}(3)-[3]
\end{gathered}
$$

is also depicted in figure 46. Like the previous case, one has another $\operatorname{SL}(2, \mathbf{Z})$ invariant 7-brane combination except for a different resolution of the $O 7^{-}$plane, $O 7^{-} \rightarrow \mathbf{N}, \mathbf{X}_{[\mathbf{2}, \mathbf{1}]}$. When performing an S-duality, the web configuration becomes completely different from the previous one as a D7 brane is being attached to a D5 brane which makes a floating 7-brane pair which can be converted into an $\mathrm{O}^{-}$plane in the weak coupling limit. One can further move this D7 brane across 5-brane junction, and as the Hanany-Witten transition, one has freely floating D7 branes, which play the role of the fundamental flavors. The resulting configuration yields a quiver theory made out of $S p$ and $S U$ gauge groups. See figure 46. Therefore, we claim that two different quiver theories have the same $6 \mathrm{~d}$ origin at UV and thus they are two dual description at IR. We note also that taking the flavor decoupling limit, this duality would still hold for less flavor cases.

$\boldsymbol{N}=$ odd: Sp - SU quiver. In a similar fashion, one can obtain $5 \mathrm{~d}$ theory for $N=$ $2 m+1$. In this case, the resolution of each $O 7^{-}$brane is different, one does get dual description, rather one finds the resulting $5 \mathrm{~d}$ theory is a hybrid of the $N=2 \mathrm{~m}$ cases:

$$
5 \mathrm{~d}[3]-\underbrace{\mathrm{Sp}(n)-\mathrm{SU}(2 n+1)-\mathrm{SU}(2 n+1)-\cdots-\mathrm{SU}(2 n+1)}_{m+1 \text { nodes }}-\left[1_{A}, 4\right] .
$$

As an example, $5 \mathrm{~d}$ brane configuration for $6 \mathrm{~d}\left[1_{A}, 8\right]-\mathrm{SU}(5)-\mathrm{SU}(5)-\mathrm{SU}(5)-[5]$ quiver theory is depicted in figure 47 , yielding

$$
5 \mathrm{~d}\left[1_{A}, 4\right]-\mathrm{SU}(7)-\mathrm{SU}^{\mid}(7)-\mathrm{Sp}(3)-[3] .
$$




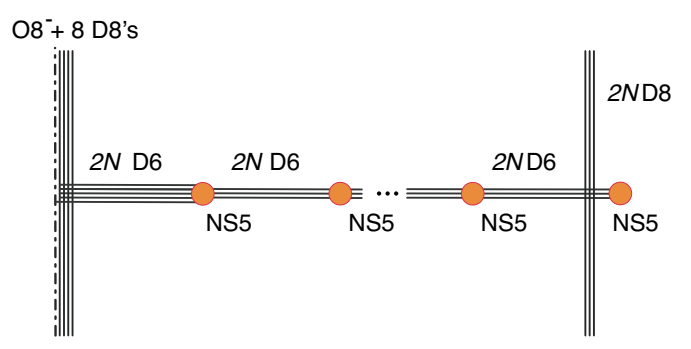

Figure 48. 6d brane configuration for the $[8]-\mathrm{Sp}(N)-\mathrm{SU}(2 N)-\mathrm{SU}(2 N)-\cdots-\mathrm{SU}(2 N)-[2 N]$ quiver theory.

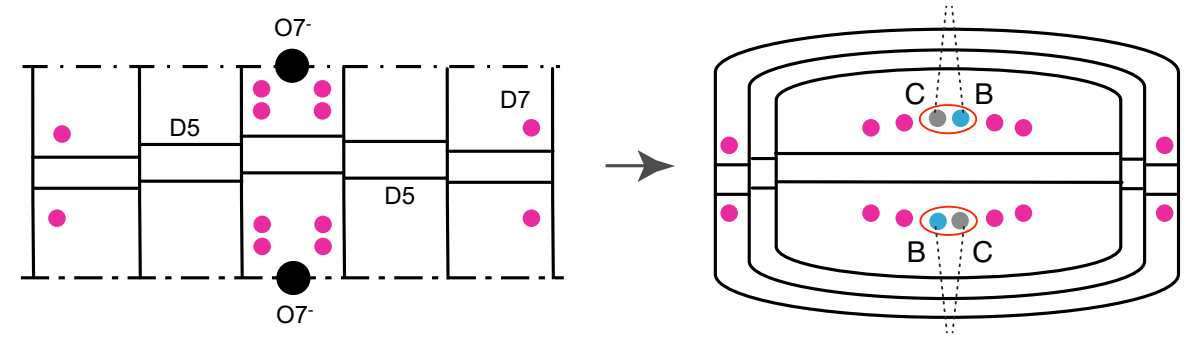

Figure 49. T-dual version of $6 \mathrm{~d}$ brane configuration for the $[8]-\mathrm{Sp}(2)-\mathrm{SU}(4)-\mathrm{SU}(4)-[4]$ quiver theory.

\section{$5.26 \mathrm{~d}[8]-\mathrm{Sp}(N)-\mathrm{SU}(2 N)-\mathrm{SU}(2 N)-\cdots-\mathrm{SU}(2 N)-[2 N]$ quiver}

Next we consider another special case:

$$
6 \mathrm{~d}[8]-\underbrace{\mathrm{Sp}(N)-\mathrm{SU}(2 N)-\mathrm{SU}(2 N)-\cdots-\mathrm{SU}(2 N)}_{n \text { nodes }}-[2 N]
$$

whose brane configuration is given in figure 48, consisting of an $\mathrm{O}^{-}$plane, and D8 branes, D6 branes and NS5 branes. We claim that from a circle compactification and T-dual, one obtains the $5 \mathrm{~d}$ theory given by

$$
5 \mathrm{~d}[4]-\underbrace{\mathrm{Sp}(n)-\mathrm{SU}(2 n)-\cdots-\mathrm{SU}(2 n)-\mathrm{Sp}(n)}_{N+1 \text { nodes }}-[4] .
$$

As an example, consider

$$
6 \mathrm{~d}[8]-\mathrm{Sp}(2)-\mathrm{SU}(4)-\mathrm{SU}(4)-[4]
$$

Reducing it to $5 \mathrm{~d}$, one gets a brane configuration of $07^{-}$planes, D7 branes and D5 branes given in the web configuration on the left of figure 49. By resolving two $\mathrm{O7}^{-}$planes, one obtains the configuration on the right of figure 49. As discussed in the beginning of this section, using the $\mathrm{SL}(2, \mathbb{Z})$ invariant 7-brane combination (5.8), one sees that S-duality leads to

$$
5 \mathrm{~d}[4]-\mathrm{Sp}(3)-\mathrm{SU}(6)-\mathrm{Sp}(3)-[4] \text {. }
$$

The procedure is depicted in figure 50 . 

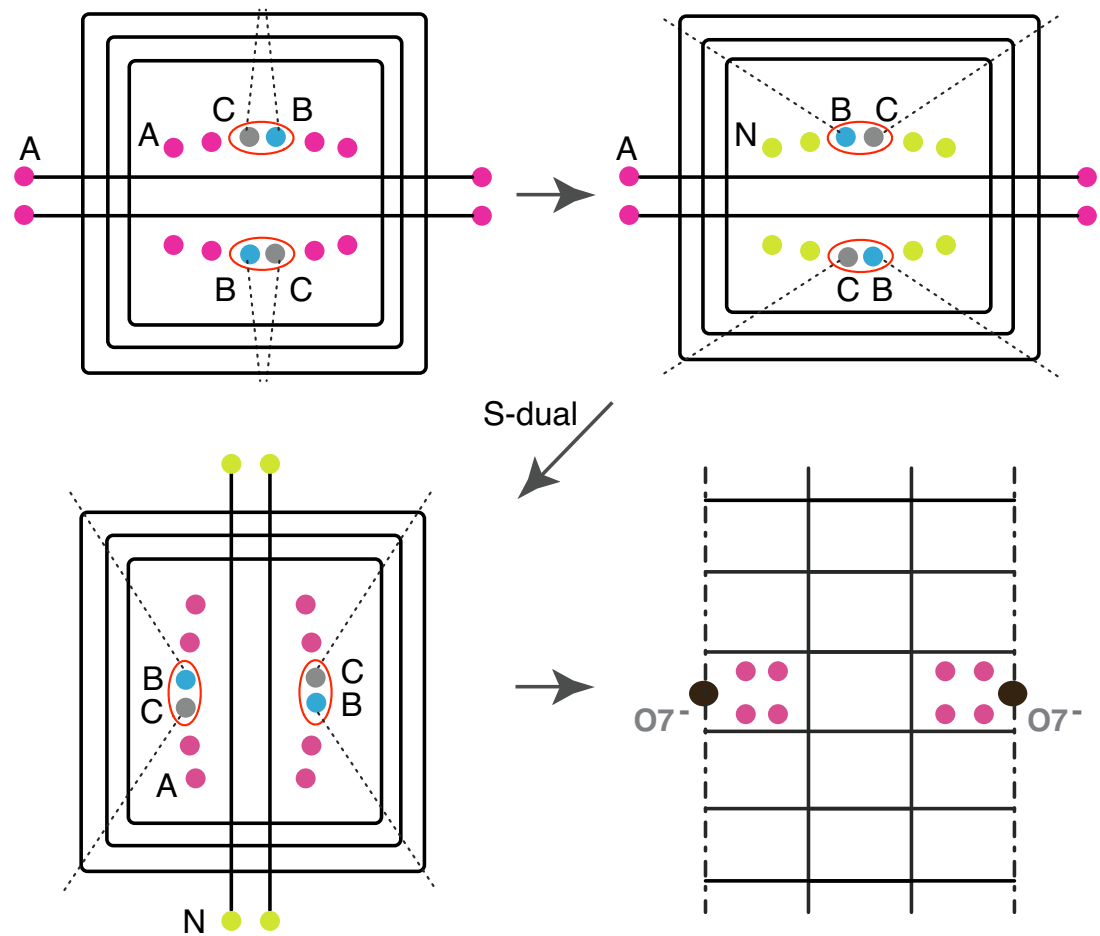

Figure 50. The first figure is a simplified version of the right of figure 49 for $6 \mathrm{~d}[8]-\mathrm{Sp}(2)-\mathrm{SU}(4)-$ $\mathrm{SU}(4)-\mathrm{SU}(4)-[4]$ quiver. The 7-branes are distributed and combined to form an S-dual invariant 7-brane combination. The second figure is the rearrangement of these 7-branes via monodromies explained in section 5.1. The third one is the result of S-dual action of the second one. The last one is the corresponding brane configuration showing $5 \mathrm{~d}[4]-\mathrm{Sp}(3)-\mathrm{SU}(6)-\mathrm{Sp}(3)-[4]$.

\section{$5.36 \mathrm{~d}[8+m]-\mathrm{Sp}(N)_{\mathrm{T}}-\mathrm{SU}(2 N-m)_{\mathrm{T}}-\mathrm{SU}(2 N-2 m)_{\mathrm{T}}-\mathrm{SU}(2 N-3 m)_{\mathrm{T}}-\ldots$ quiver}

As a generalization of section 5.2 , we consider $m>0$ :

$$
6 d \quad[8+m]-\mathrm{Sp}(N)_{\mathrm{T}}-\mathrm{SU}(2 N-m)_{\mathrm{T}}-\mathrm{SU}(2 N-2 m)_{\mathrm{T}}-\mathrm{SU}(2 N-3 m)_{\mathrm{T}}-\ldots
$$

\section{$6 \quad 6 d$ description of $5 \mathrm{~d} T_{N}$ Tao theory}

There is another important class of $5 \mathrm{~d}$ theories obtained by Tao diagrams. It has been known that the $5 \mathrm{~d} T_{N}$ theory can be realized by a 5 -brane web diagram [42]. By turning on certain mass deformations, the $5 \mathrm{~d} T_{N}$ theory flows to a $5 \mathrm{~d}$ linear quiver theory $[N]-$ $\mathrm{SU}(N-1)-\mathrm{SU}(N-2)-\cdots-\mathrm{SU}(2)-\mathrm{SU}(1)$ [43-46]. The last $\mathrm{SU}(1)$ node can be understood as two flavors coupled to the $\mathrm{SU}(2)$ gauge node, and hence the quiver is equivalently written as $[N]-\mathrm{SU}(N-1)-\mathrm{SU}(N-2)-\cdots-\mathrm{SU}(2)-[2][32,45,46]$. It is possible to construct a Tao web diagram by adding flavors to the two end nodes of the quiver [30]. An example of a Tao web diagram arising by adding flavors to the $5 \mathrm{~d} T_{5}$ theory is depicted in figure 51 . The $5 \mathrm{~d}$ quiver theory realized by the Tao diagram is then

$$
[N+2]-\mathrm{SU}(N-1)-\mathrm{SU}(N-2)-\cdots-\mathrm{SU}(3)-\mathrm{SU}(2)-[3] .
$$




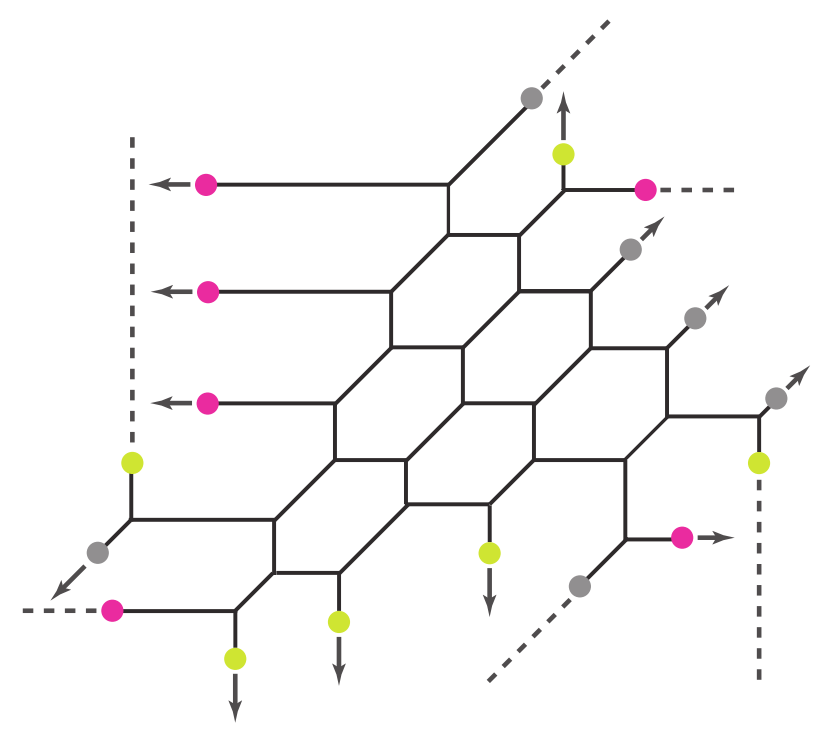

Figure 51. The Tao web diagram which arises by adding flavors at the end nodes of the 5 d quiver corresponding to the $5 \mathrm{~d} T_{5}$ theory.

We will call the $5 \mathrm{~d}$ quiver theory as $5 \mathrm{~d} T_{N}$ Tao theory. Since the $5 \mathrm{~d} T_{N}$ Tao theory is realized by the Tao diagram, the theory is expected to have a $6 \mathrm{~d}$ UV completion. In this section, we propose a $6 \mathrm{~d}$ description of the $5 \mathrm{~d} T_{N}$ Tao theory (6.1) realized by the $T_{N}$ Tao diagram by examining the global symmetry as well as the number of the Coulomb branch moduli.

\subsection{Global symmetry from 7-branes}

The global symmetry of a $5 \mathrm{~d}$ theory realized by a 5 -brane web can be understood from the symmetry on 7-branes attached to external 5-branes in the web diagram [37]. Namely, 7-branes can determine the global symmetry realized at the UV fixed point. In order to see the flavor symmetry, we pull all the 7-branes inside the 5-brane loops and try to put 7-branes on top of each other. The symmetry realized on 7-branes gives the non-Abelian part of the flavor symmetry of the $5 \mathrm{~d}$ theory. Abelian part can be recovered by counting the number of parameters of the theory since the number of the parameters of the $5 \mathrm{~d}$ theory should agree with the rank of the total global symmetry. The method was applied to the Tao diagram realizing the $5 \mathrm{~d} \mathrm{SU}(n)$ gauge theory with $N_{f}=2 n+4$ flavors in [27], and the global symmetry was determined to be $\mathrm{SO}(4 n+8)$. We repeat the same procedure to determine the global symmetry of the $5 \mathrm{~d} T_{N}$ Tao theory.

We first pull all the 7 -branes inside the 5 -brane loops. Since $[p, q] 7$-branes mutually commute with $(p, q) 5$-branes, it is possible to collect the 7-branes into three chambers, the top-left one (1st chamber), the bottom-left one (2nd chamber) and the bottom-right one (3rd chamber) as in figure 52. The 7-branes in each chamber are schematically summarized as

$$
\mathbf{A N C A}^{N-2}\left\|\mathbf{N C A N}^{N-2}\right\| \mathbf{C A N C}^{N-2} \|
$$

where $\mathbf{A}, \mathbf{C}$ and $\mathbf{N}$ represent $[1,0],[1,1]$ and $[0,1]$ 7-branes respectively. We will also express $[1,-1]$-brane by $\mathbf{B}$. In this expression of (6.2), the branch cuts are assumed 


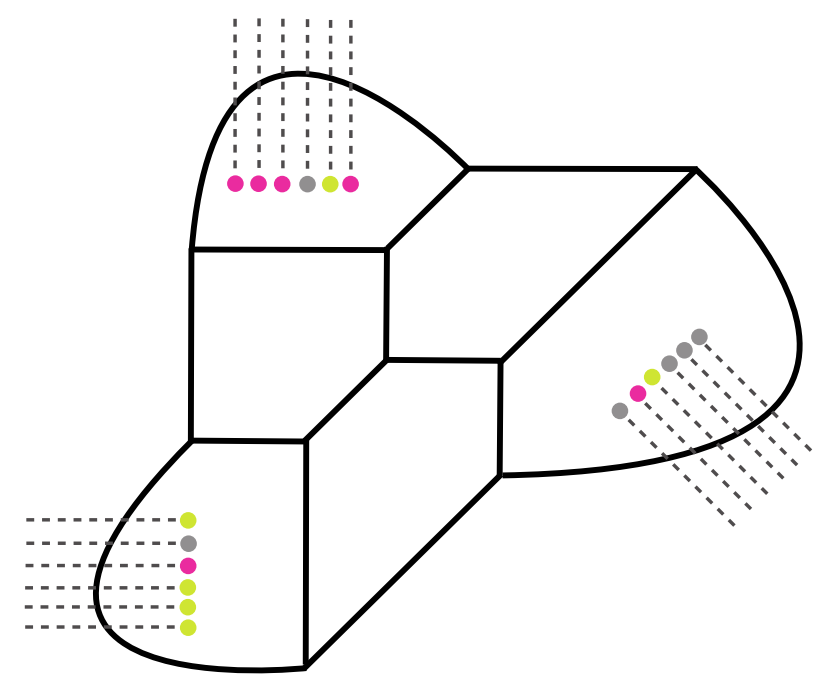

Figure 52. The 5-branes web after pulling all the 7-branes inside the 5-brane loops. We write an example of the $5 \mathrm{~d} T_{5}$ Tao theory as an example.

to extend in the lower direction, and the charge of a 7-brane change by the monodromy matrix

$$
\left(\begin{array}{cc}
1-p q & p^{2} \\
-q^{2} & 1+p q
\end{array}\right)
$$

when the 7-brane crosses the branch cut of a $[p, q] 7$-brane counterclockwise. Also, the first, the second and the third partition lines in (6.2) represent $(1,0) 5$-branes, $(0,1) 5$-branes and $(1,1) 5$-branes respectively. Hence, 7 -branes with the same charge as the 5-brane corresponding to the partition line can crosse its line. Therefore, we can move 7 -branes in (6.2), and obtain

$$
\mathbf{C}^{N-2} \mathbf{A N C}\left\|\mathbf{A}^{N-2} \mathbf{N C A N} \mathbf{N}^{N-2}\right\| \mathbf{C A N} \|
$$

By rearrangement of 7-branes inside each chamber, the 7-brane configuration becomes

$$
\mathbf{X}_{[2,1]} \mathbf{A}^{N}\left\|\mathbf{A}^{2 N-2} \mathbf{B}\right\| \mathbf{N}^{2} \mathbf{X}_{[2,1]} \|
$$

By moving 7-branes between the chambers with the rearrangemnt in the second chamber, we finally obtain

$$
\mathbf{X}_{[2,1]}\left\|\mathbf{A}^{3 N} \mathbf{B}\right\| \mathbf{X}_{[2,1]} \|
$$

The presence of $3 \mathrm{~N}$ A-branes on top of each other means that the non-Abelian part of the global symmetry of the $5 \mathrm{~d} T_{N}$ Tao theory is $\mathrm{SU}(3 N)$ symmetry.

It is possible to count the number of the parameters of the $5 \mathrm{~d} T_{N}$ Tao theory. The number of mass parameters of the fundamental hypermultiplets is $N+5$. The number of mass parameters of the bi-fundamental multiplets is $N-3$. Also, the number of the gauge couplings or the mass parameters for the instantons is $N-2$. The total number is $3 N$. Therefore, the full global symmetry of the $5 \mathrm{~d}$ theory is $\mathrm{SU}(3 N) \times \mathrm{U}(1)_{I}$. As expected, we have the $\mathrm{U}(1)_{I}$ symmetry associated to the Kaluza-Klein modes from the $S^{1}$ compactification of a $6 \mathrm{~d}$ theory. 
The dimension of the Coulomb branch moduli space can be determined easily by counting the rank of the gauge groups, and it is $\frac{(N-1)(N-2)}{2}$.

\section{2 $5 \mathrm{~d} T_{4}, T_{5}$ and $T_{6}$ Tao theories}

Let us move on to a candidate for a $6 \mathrm{~d}$ description of the $5 \mathrm{~d} T_{N}$ Tao theory. We first focus on the $5 \mathrm{~d} T_{4}, T_{5}, T_{6}$ theories, which will be basic examples for the general $5 \mathrm{~d} T_{N}$ Tao theory.

In fact, the $5 \mathrm{~d} T_{4}$ Tao theory is a very special case in the examples considered in section 3. The $6 \mathrm{~d}$ description in the tensor branch is

$$
[1]_{A}-\mathrm{SU}(3)-[11]
$$

We also have one tensor multiplet corresponding to the number of the gauge nodes. The theory has one tensor multiplet and two vector multiplets in the Cartan subalgebra, and hence the sum of them reproduces the correct number of the 5 d vector multiplets in the Cartan subalgebra of the $5 \mathrm{~d} T_{4}$ theory.

Let us then determine the flavor symmetry of the $6 \mathrm{~d}$ theory (6.7). It is important to note that the anti-symmetric representation of the $\mathrm{SU}(3)$ is equivalent to the antifundamental representation of the $\mathrm{SU}(3)$. Although a $6 \mathrm{~d}$ hypermultiplet contains a $6 \mathrm{~d}$ Weyl spinor, the complex conjugation of the $6 \mathrm{~d}$ Weyl spinor does not change its chirality. Therefore, the hypermultiplet in the anti-symmetric representation of $\mathrm{SU}(3)$ is equivalent to a hypermultiplet in the fundamental representation of SU(3). Namely, the $6 \mathrm{~d}$ theory (6.7) is equivalent to a $6 \mathrm{~d} \mathrm{SU}(3)$ gauge theory with 12 flavors with one tensor multiplet coupled. From this point of view, we have at least an SU(12) flavor symmetry.

There can be a potential U(1) flavor symmetry that may come from the overall part of $\mathrm{U}(12)$. However, we argue that the $\mathrm{U}(1)$ symmetry is broken by the anomaly $\mathrm{U}(1)_{\text {global }}-$ $\mathrm{SU}(3)_{\text {gauge }}^{3}$, and does not appear as a global symmetry of the $6 \mathrm{~d}$ theory. Since the thirdorder Casimir invariant for $\mathrm{SU}(3)$ is non-trivial, it is impossible to cancel the anomaly if there is only one representation. Therefore, the $6 \mathrm{~d}$ theory does not have an Abelian symmetry.

After the circle compactification, the KK mode provides a $\mathrm{U}(1)_{I}$ symmetry. Hence, the total flavor symmetry is $\mathrm{SU}(12) \times \mathrm{U}(1)_{I}$, which agrees with the flavor symmetry of the $5 \mathrm{~d} T_{4}$ Tao theory.

Let us then consider the $5 \mathrm{~d} T_{5}$ Tao theory. In this case, it is not included in the examples we have discussed so far. This means that it may not admit a brane configuration in Type IIA string theory. However, it has been conjectured that all the 6d SCFTs may be realized by F-theory compactifications $[1,3-5]$. Then it is possible to find a candidate for its 6d description in the $6 \mathrm{~d}$ SCFTs classified by the F-theory compactifications, by comparing the global symmetry as well as the dimension of the Coulomb branch moduli space.

In order to realize a 6d SCFT from F-theory, we consider an F-theory compactification on a Calabi-Yau threefold which is given by an elliptically fibration over a non-compact complex two dimensional Kähler manifold $B$. In the base $B$, there is a bunch of $\mathbb{P}^{1}$ 's. Each size of the compact $\mathbb{P}^{1}$ corresponds to a vev of a scalar in a tensor multiplet. The elliptic fibration over the $\mathbb{P}^{1}$ can be singular, meaning that 7 -branes wrap the $\mathbb{P}^{1}$ and yielding a 
gauge symmetry. When all the compact $\mathbb{P}^{1}$ 's vanish simultaneously, the $6 \mathrm{~d}$ theory is at the superconformal fixed point. This in fact constrains the shape of the sequence of the $\mathbb{P}^{1}$ 's significantly $[1,5]$.

As for the $5 \mathrm{~d} T_{5}$ Tao theory, the dimension of the Coulomb branch moduli space is 6 . Therefore, the sum of the number of the tensor multiplets and the number of the vector multiplets in the Cartan subalgebra of the corresponding $6 \mathrm{~d}$ theory must be 6 . Since the expected $6 \mathrm{~d}$ flavor symmetry is $\mathrm{SU}(15)$, we need one non-compact $\mathbb{P}^{110}$ with a singular elliptic fiber giving the $\mathrm{SU}(15)$ algebra on it. We denote the non-compact 2-cycle by $C_{\mathrm{SU}(15)}$. Due to the strong constraint of the shape of the base geometry, only pairwise intersections are allowed. Furthermore, the non-trivial gauge algebra on the adjacent $\mathbb{P}^{1}$ should be either $S U$ or $S p[5]$.

Let us then consider the case where one compact $\mathbb{P}^{1}$ is attached to $C_{\mathrm{SU}(15)}$. The theory has one tensor multiplet. Hence, the rank of the $S U$ or $S p$ gauge group should be 5 . Then, the $6 \mathrm{~d}$ anomaly cancellation condition with the 15 flavors gives us a unique possibility

$$
\left[\frac{1}{2}\right]_{\Lambda^{3}}-\mathrm{SU}(6)-[15]
$$

where $\left[\frac{1}{2}\right]_{\Lambda^{3}}$ stands for one half-hypermultiplet in the rank three anti-symmetric representation. This $6 \mathrm{~d}$ theory on $S^{1}$ reproduces the correct number of the $5 \mathrm{~d}$ vector multiplets in the Cartan subalgebra of the $5 \mathrm{~d} T_{5}$ Tao theory as well as the SU(15) flavor symmetry. The potential U(1) flavor symmetry which may come from the overall part of the U(15) is again broken by the anomaly $\mathrm{U}(1)-\mathrm{SU}(6)^{3}$.

When one increases the number of compact $\mathbb{P}^{1}$ 's attached to $C_{\mathrm{SU}(15)}$, then one cannot find a candidate for a non-trivial gauge group on the compact $\mathbb{P}^{1}$ 's that satisfies the $6 \mathrm{~d}$ gauge anomaly cancellation condition with the 15 flavors. If we increase the number of the compact $\mathbb{P}^{1}$ 's, the total rank of the gauge groups should be less than or equal to 4 . Then, there is no possible choice for the gauge group on the compact $\mathbb{P}^{1}$ next to $C_{\mathrm{SU}(12)}$ by the $6 \mathrm{~d}$ anomaly cancellation condition. If there is no gauge symmetry on the compact $\mathbb{P}^{1}$ next to $C_{\mathrm{SU}(15)}$, then the 15 flavors on $C_{\mathrm{SU}(15)}$ may give a $\mathrm{U}(15)$ flavor symmetry at least. ${ }^{11}$ Therefore, it contradicts the flavor symmetry of the $5 \mathrm{~d} T_{5}$ Tao theory.

Hence, we propose that the $6 \mathrm{~d}$ description of the $5 \mathrm{~d} T_{5}$ Tao theory is given by the $6 \mathrm{~d}$ quiver of (6.8).

The last case is the $5 \mathrm{~d} T_{6}$ Tao theory. This is also a very special case in the examples considered in section 4.2. The $6 \mathrm{~d}$ description in the tensor branch is

$$
\mathrm{SU}(1)-\mathrm{SU}(9)-[17]
$$

Note that there is no hypermultiplet in the anti-symmetric representation for the $\mathrm{SU}(1)$. Furthermore, the SU(1) does not a dynamical vector multiplet. Therefore, the $6 \mathrm{~d}$ theory is effectively described by the $\mathrm{SU}(9)$ gauge theory with 18 hypermultiplets in the fundamental

\footnotetext{
${ }^{10}$ We call non-compact $\mathbb{P}^{1}$ as a $\mathbb{P}^{1}$ whose size is taken to be infinity.

${ }^{11}$ For example, the E-string theory on the tensor branch consists of 8 hypermultiplets and one tensor multiplet, The flavor symmetry is $\mathrm{SO}(16)$ which is further enhanced to $E_{8} \supset \mathrm{SO}(16)$.
} 
representation with two tensor multiplets coupled. Therefore, after the circle compactification, it gives $2+8=105 \mathrm{~d}$ vector multiplets in the Cartan subalgebras, which reproduces the correct number for the $5 \mathrm{~d} T_{6}$ Tao theory. Furthermore, the flavor symmetry is $\mathrm{SU}(18) \times$ $\mathrm{U}(1)_{I}$, which also agrees with the flavor symmetry of the $5 \mathrm{~d} T_{6}$ Tao theory. The potential $\mathrm{U}(1)$ symmetry of the overall part of $\mathrm{U}(18)$ is again broken by the anomaly $\mathrm{U}(1)-\mathrm{SU}(9)^{3}$.

\subsection{Generalization}

We move on to the analysis for a $6 \mathrm{~d}$ description of the $5 \mathrm{~d} T_{N}$ Tao theory. Given the $6 \mathrm{~d}$ description of the $5 \mathrm{~d} T_{4}, T_{5}, T_{6}$ Tao theory, there is a very natural generalization to a general $N$.

The $6 \mathrm{~d}$ description of the $5 \mathrm{~d} T_{4}$ Tao theory is described by the $6 \mathrm{~d} \mathrm{SU}(3)$ gauge theory with 12 flavors, and it has the SU(12) flavor symmetry. We then gauge the SU(12) flavor symmetry. Due to the $6 \mathrm{~d}$ anomaly cancellation condition, the $\mathrm{SU}(12)$ gauge node needs to have 12 more flavors. Namely, a 6d anomaly free quiver theory by gauging the SU(12) flavor symmetry is

$$
\mathrm{SU}(3)-\mathrm{SU}(12)-[21]
$$

It has two tensor multiplets and 13 vector multiplets in the Cartan subalgebra. The total number is 15, and this number in fact agrees with the number of the Coulomb branch moduli of the $5 \mathrm{~d} T_{7}$ theory. Furthermore, the flavor symmetry of the $6 \mathrm{~d}$ theory (6.10) after the $S^{1}$ compactification is $\mathrm{SU}(21) \times \mathrm{U}(1)_{I}$, which also agrees with the global symmetry of the $5 \mathrm{~d} T_{7}$ Tao theory. In this case, there can be potential $\mathrm{U}(1) \times \mathrm{U}(1)$ flavor symmetries. One may be associated to the one bi-fundamental hypermultiplet between $\mathrm{SU}(3)$ and $\mathrm{SU}(12)$, and the other may be associated to the 21 fundamental hypermultiplets. However, any linear combination of the two U(1)'s has non-zero anomaly either from $\mathrm{U}(1)-\mathrm{SU}(3)^{3}$ or $\mathrm{U}(1)-\mathrm{SU}(12)^{3}$. Therefore, the $6 \mathrm{~d}$ theory (6.10) is a natural candidate for the $5 \mathrm{~d} T_{7}$ theory.

It is easy to generalize this consideration by gauging the $\mathrm{SU}(21)$ flavor symmetry. The general quiver theory by the successive gauging is

$$
\mathrm{SU}(3)-\mathrm{SU}(12)-\mathrm{SU}(21)-\cdots-[9 N+3] .
$$

It has $N$ tensor multiplets and $\frac{N(9 N-5)}{2}$ vector multiplets in the Cartan subalgebra. The total number is $\frac{3 N(3 N-1)}{2}$, which exactly agrees with the number of the Coulomb branch moduli of the $5 \mathrm{~d} T_{3 N+1}$ Tao theory. The flavor symmetry after the $S^{1}$ compactification is $\mathrm{SU}(9 M+3) \times \mathrm{U}(1)_{I}$, which also agrees with that of the $5 \mathrm{~d} T_{3 N+1}$ Tao theory. The potential $\mathrm{U}(1)^{N}$ flavor symmetries are all broken by the [global - gauge ${ }^{3}$ ] anomalies. Hence, the $6 \mathrm{~d}$ quiver theory (6.11) is a candidate for the $6 \mathrm{~d}$ description of the $5 \mathrm{~d} T_{3 N+1}$ Tao theory with $N \geq 1$.

It is interesting to note that the rank of the gauge group of the $6 \mathrm{~d}$ quiver (6.11) increases by 9 . Hence, this $6 \mathrm{~d}$ theory is not realized by a brane configuration with an $O 8^{-}$-plane in Type IIA string theory. However, it may admit a F-theory realization, and a possible configuration may be

$$
\begin{array}{ccccc}
\mathfrak{s u}(3) & \mathfrak{s u}(12) & \cdots & \mathfrak{s u}(9 N-6) & {[\mathfrak{s u}(9 N+3)]} \\
1 & 2 & \cdots & 2
\end{array}
$$


Each column represents a $\mathbb{P}^{1}$ and the last one stands for a non-compact $\mathbb{P}^{1}$ in the base $B$. The upper row indicates the singular type of the elliptic fibration over the $\mathbb{P}^{1}$. The lower row gives the self-intersection number of each compact $\mathbb{P}^{1}$ inside $B$.

In fact, the proposed $6 \mathrm{~d}$ descriptions of the $5 \mathrm{~d} T_{5}$ and $T_{6}$ Tao theories also admit the same generalization. By the successive gauging of the flavor symmetries of (6.8), one arrives at

$$
\left[\frac{1}{2}\right]_{\Lambda^{3}}-\mathrm{SU}(6)-\mathrm{SU}(15)-\cdots-\mathrm{SU}(9 N-3)-[9 N+6] .
$$

The 6d quiver theory has $N$ tensor multiplets and $\frac{N(9 N+1)}{2}$ vector multiplets in the Cartan subalgebra. The sum of the numbers give $\frac{3 N(3 N+1)}{2}$, which is exactly the dimension of the Coulomb branch moduli space of the $5 \mathrm{~d} T_{3 N+2}$ Tao theory with $N \geq 1$. The potential $\mathrm{U}(1)^{N}$ global symmetries are all broken by the [global - gauge ${ }^{3}$ ] anomaly of some gauge group. Hence, the flavor symmetry after the circle compactification is $\mathrm{SU}(9 N+6) \times \mathrm{U}(1)_{I}$. and it agrees with that of the $5 \mathrm{~d} T_{3 N+2}$ Tao theory. Therefore, the $6 \mathrm{~d}$ quiver theory (6.12) is a natural candidate of the $6 \mathrm{~d}$ description of the $5 \mathrm{~d} T_{3 N+2}$ Tao theory.

Again, the 6d quiver theory (6.12) does not have a Type IIA bran realization, but it may have a F-theory realization. The possible configuration is

$$
\begin{array}{ccccc}
\mathfrak{s u}(6) & \mathfrak{s u}(15) & \cdots & \mathfrak{s u}(9 N-3) & {[\mathfrak{s u}(9 N+6)]} \\
1 & 2 & \cdots & 2
\end{array}
$$

where the first $\mathbb{P}^{1}$ can support the half-hypermultiplet in the rank three anti-symmetric representation [5].

Let us finally turn to the generalization of the $5 \mathrm{~d} T_{6}$ theory by gauging the flavor symmetry. The $6 \mathrm{~d}$ theory is a quiver theory described by

$$
\mathrm{SU}(0)-\mathrm{SU}(9)-\mathrm{SU}(18)-\cdots-\mathrm{SU}(9 N)-[9 N+9] .
$$

The first SU(0) indicates an additional tensor multiplet without any gauge dynamics. The $6 \mathrm{~d}$ theory has $N+1$ tensor multiplets and $\frac{N(9 N+7)}{2}$. The sum of the numbers is $\frac{(3 N+2)(3 N+1)}{2}$, which agrees with the number of the Coulomb branch moduli of the $5 \mathrm{~d} T_{3 N+3}$ theory. Due to the [global - gauge ${ }^{3}$ ] anomalies, the potential $\mathrm{U}(1)^{N}$ global symmetries are all broken. Hence, the flavor symmetry after the $S^{1}$ compactification is $\mathrm{SU}(9 N+9) \times \mathrm{U}(1)_{I}$, and it correctly reproduces the global symmetry of the $5 \mathrm{~d} T_{3 N+3}$ Tao theory. The possible F-theory realization of the $6 \mathrm{~d}$ theory is

$$
\begin{array}{lclcc}
\emptyset & \mathfrak{s u}(9) & \cdots & \mathfrak{s u}(9 N) & {[\mathfrak{s u}(9 N+9)]} \\
1 & 2 & \cdots & 2
\end{array}
$$

where the first $\mathbb{P}^{1}$ does not support a gauge algebra.

So far we have considered the $5 \mathrm{~d} T_{3 N+3}$ Tao theory with $N \geq 1$. It is interesting to think of the case where $N=0$. This case admits a Type IIA brane configuration that was considered in section 5 . The $6 \mathrm{~d}$ quiver description is

$$
\mathrm{SU}(1)-[9] .
$$


In fact, this theory has been shown to be equivalent to the rank 1 E-string theory in section 5. This is perfect agreement with the formal substitution of $N=0$ into the $5 \mathrm{~d}$ $T_{3 N+3}$ Tao theory since the $5 \mathrm{~d} T_{3}$ Tao theory is nothing but the rank 1 E-string theory [30].

\section{Discussions}

We have started with $6 \mathrm{~d} \mathcal{N}=(1,0)$ superconformal field theories whose Type IIA brane representations are made of NS5-branes, D6-branes, D8-branes and a single O8 $^{-}$-plane. On their tensor branch, they are in general written as a linear quiver diagram with various matter hypermultiplets. After a circle compactification and T-duality, we have found a very rich group of $5 \mathrm{~d} \mathcal{N}=1$ gauge theories that are dual to each other. This diversity of the $5 \mathrm{~d}$ theories for a given $6 \mathrm{~d}$ theory originates from the choice in the resolution of two $O 7^{-}$planes and in the distribution of D5 and D7-branes as well as a part of the $\operatorname{SL}(2, \mathbb{Z})$ duality. A decoupling of the same flavors from the dual 5 d theories leads to another duality between $5 \mathrm{~d}$ gauge theories which would have the identical $5 \mathrm{~d}$ superconformal field theory.

While we provided the argument for the duality between $5 \mathrm{~d}$ gauge theories by starting with the brane picture of their $6 \mathrm{~d}$ mother theory, one can have different class of $6 \mathrm{~d}$ superconformal field theories whose brane picture is not in the class considered here. There are also some $6 \mathrm{~d}$ superconformal field theories with no obvious brane picture. It would be interesting to consider their $5 \mathrm{~d}$ counterparts and the corresponding $5 \mathrm{~d}$ dualities.

\section{Acknowledgments}

We thank Kang-Sin Choi, Hee-Cheol Kim, Seok Kim, Xiao Liu, Soo-Jong Rey, Ashoke Sen and Piljin Yi for useful discussions. We especially thank Masato Taki for early collaboration and discussions. We are thankful to the Workshop on Geometric Correspondences of Gauge Theories at SISSA. S.K. is grateful to "APCTP focus program on holography and its application" (2015) where a part of work is done. The work of H.H. is supported by the grant FPA2012-32828 from the MINECO, the REA grant agreement PCIG10-GA-2011304023 from the People Programme of FP7 (Marie Curie Action), the ERC Advanced Grant SPLE under contract ERC-2012-ADG-20120216-320421 and the grant SEV-20120249 of the "Centro de Excelencia Severo Ochoa" Programme. The work of HH is also supported in part by Perimeter Institute for Theoretical Physics where a part of the work was done. Research at Perimeter Institute is supported by the Government of Canada through Industry Canada and by the Province of Ontario through Ministry of Economic Development \& Innovation. The work of K.L. is supported in part by the National Research Foundation of Korea (NRF) Grants No. 2006-0093850.

\section{A A brief introduction of Tao diagram}

A Tao diagram is a generalization of $5 \mathrm{~d}(p, q)$ web diagram with the critical number of flavors that gives the UV completion as $6 \mathrm{~d}$ theory. A typical example is $5 \mathrm{~d} \mathrm{SU}(2)$ superconformal theory of $N_{f}=8$ flavors, which is understood as a circle compactification of $6 \mathrm{~d}$ 


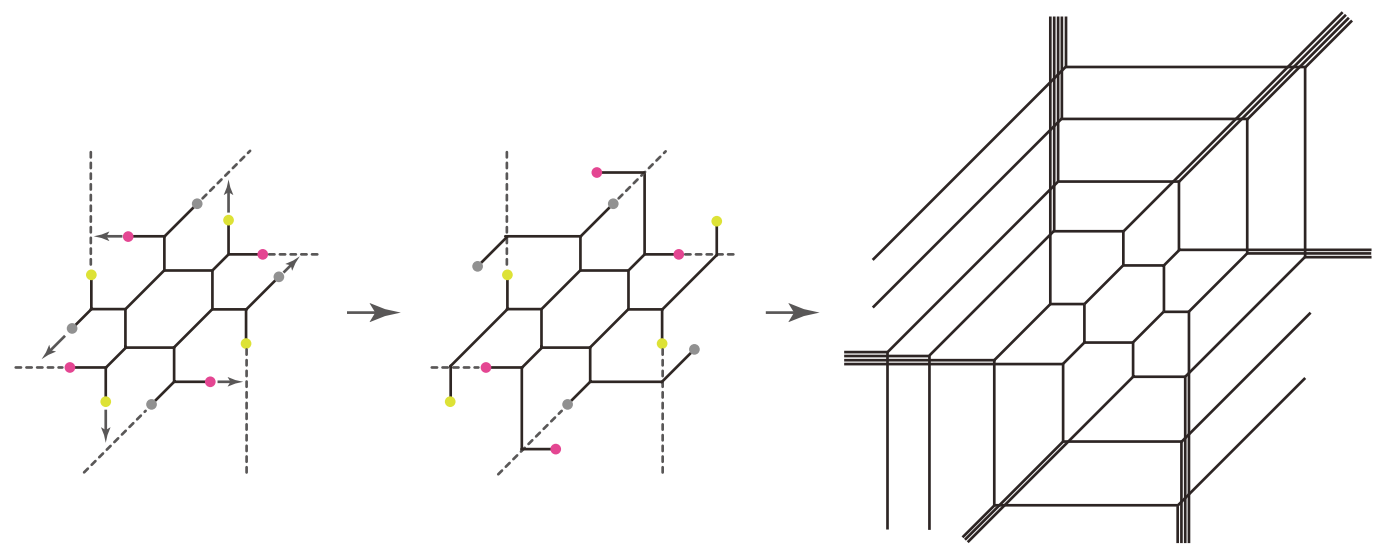

Figure 53. An example of Tao diagram for $5 \mathrm{~d} \mathrm{SU}(2)$ theory of $N_{f}=8$. There are twelve 7 -branes of various charges which are the end point of 5-branes of the same charge. The colored dots denote 7-branes and different colors means different charges. Middle: six branch cuts are chosen such that other remaining 7-branes are supposed to cross as being pulled to infinity. Right: the charges of 7-branes are altered as they crossed the branch cut. A repeated process generates an infinite spiral web. Right: the last step is to pull out 7-branes associated with the branch cuts. In so doing, the Hanany-Witten transition creates 5-branes along the direction that the 7-branes are pulled out such that the newly created 5-branes are bound by one 7-brane. The resulting diagram makes a Tao diagram which possesses a constant period giving rise to KK mode, and infinite web diagram corresponding to infinite KK spectrum so that the Tao diagram describes a circle compactification of $6 \mathrm{~d}$ SCFT.

E-string theory. Note that the theories of $N_{f} \leq 7$ have the 5d UV fixed point [47] while the $N_{f}=8$ case has $6 \mathrm{~d}$ UV fixed point. The corresponding $(p, q)$ web configuration has different features than the cases with $N_{f} \leq 7$. Salient features are that the web diagram form as periodic web configuration in a spiral whose period is identified as the instanton factor (squared) of the theory which is in turns identified as the KK mode of the circle compactified $6 \mathrm{~d}$ theory. Infinite spirals correspond to an infinite KK spectrum coming from the compactification on a circle. The shape resembles a Taoism symbol which is why it is named to be Tao diagram. Suitable 7-brane monodromies give various yet equivalent diagrams, one of which can be made out of $(1,0),(0,1)$ and $(1,1) 5$-brane charges, which enables one to compute the partition function via the topological vertex technique. As for $\mathrm{SU}(2)$ gauge theory of $N_{f}=8$ flavors, an explicit computation [30] was done and reproduced the result of the E-string elliptic genus computation [10]. This confirms that the Tao diagram indeed describes a circle compactification of the 6d E-string theory.

To see how infinite spiral shape arises, one first chooses some of the 7-brane branch cuts, and then pulls out other 7-branes along the geodesic direction given by the charge of 7-brane in the $(p, q)$ web plane. As 7-branes cross the branch cuts, the charges of the 7 -branes change according to the monodromy associated with the branch cut. For instance, see figure 53. When the number of flavors is critical, all the pulled-out 7-branes inevitably cross all the chosen branch cuts so that the charge of a pulled-out 7-brane becomes the same after one revolution making a spiral shape of a constant separation, or a constant period. The resulting diagram is a web diagram of infinite spiral. 
The constant period is expressed in terms of the instanton factor of the $5 \mathrm{~d}$ theory. It is expected for a circle compactification of a $6 \mathrm{~d}$ theory, and the constant period can be identified with the KK mode of the compactification. Hence, it possesses a compactified circle, more precisely, the radius of the T-dual circle.

For higher rank gauge theory or quiver theories, the configuration is basically same as shown in the Tao diagrams in the main text.

Tao web diagrams also give a computational tool using topological vertex methods.

Open Access. This article is distributed under the terms of the Creative Commons Attribution License (CC-BY 4.0), which permits any use, distribution and reproduction in any medium, provided the original author(s) and source are credited.

\section{References}

[1] J.J. Heckman, D.R. Morrison and C. Vafa, On the classification of 6D SCFTs and generalized ADE orbifolds, JHEP 05 (2014) 028 [Erratum ibid. 06 (2015) 017] [arXiv: 1312.5746] [INSPIRE].

[2] D. Gaiotto and A. Tomasiello, Holography for $(1,0)$ theories in six dimensions, JHEP 12 (2014) 003 [arXiv: 1404.0711] [INSPIRE].

[3] M. Del Zotto, J.J. Heckman, A. Tomasiello and C. Vafa, 6d conformal matter, JHEP 02 (2015) 054 [arXiv: 1407.6359] [INSPIRE].

[4] J.J. Heckman, More on the matter of 6D SCFTs, Phys. Lett. B 747 (2015) 73 [arXiv: 1408.0006] [INSPIRE].

[5] J.J. Heckman, D.R. Morrison, T. Rudelius and C. Vafa, Atomic classification of 6D SCFTs, Fortsch. Phys. 63 (2015) 468 [arXiv:1502.05405] [INSPIRE].

[6] L. Bhardwaj, Classification of $6 d N=(1,0)$ gauge theories, JHEP 11 (2015) 002 [arXiv: 1502.06594] [INSPIRE].

[7] B. Haghighat, A. Iqbal, C. Kozçaz, G. Lockhart and C. Vafa, M-strings, Commun. Math. Phys. 334 (2015) 779 [arXiv:1305.6322] [InSPIRE].

[8] B. Haghighat, C. Kozcaz, G. Lockhart and C. Vafa, Orbifolds of M-strings, Phys. Rev. D 89 (2014) 046003 [arXiv: 1310.1185] [INSPIRE].

[9] B. Haghighat, G. Lockhart and C. Vafa, Fusing E-strings to heterotic strings: $E+E \rightarrow H$, Phys. Rev. D 90 (2014) 126012 [arXiv: 1406.0850] [inSPIRE].

[10] J. Kim, S. Kim, K. Lee, J. Park and C. Vafa, Elliptic genus of E-strings, JHEP 09 (2017) 098 [arXiv: 1411.2324] [INSPIRE].

[11] B. Haghighat, A. Klemm, G. Lockhart and C. Vafa, Strings of minimal 6d SCFTs, Fortsch. Phys. 63 (2015) 294 [arXiv: 1412.3152] [INSPIRE].

[12] A. Gadde, B. Haghighat, J. Kim, S. Kim, G. Lockhart and C. Vafa, 6d string chains, JHEP 02 (2018) 143 [arXiv: 1504.04614] [INSPIRE].

[13] B. Haghighat, S. Murthy, C. Vafa and S. Vandoren, F-theory, spinning black holes and multi-string branches, JHEP 01 (2016) 009 [arXiv: 1509.00455] [INSPIRE].

[14] K. Ohmori, H. Shimizu and Y. Tachikawa, Anomaly polynomial of E-string theories, JHEP 08 (2014) 002 [arXiv: 1404.3887] [INSPIRE]. 
[15] K. Ohmori, H. Shimizu, Y. Tachikawa and K. Yonekura, Anomaly polynomial of general $6 d$ SCFTs, PTEP 2014 (2014) 103B07 [arXiv: 1408.5572] [INSPIRE].

[16] K. Intriligator, $6 d, N=(1,0)$ Coulomb branch anomaly matching, JHEP 10 (2014) 162 [arXiv: 1408.6745] [INSPIRE].

[17] J.J. Heckman, D.R. Morrison, T. Rudelius and C. Vafa, Geometry of 6D RG flows, JHEP 09 (2015) 052 [arXiv: 1505.00009] [INSPIRE].

[18] J.J. Heckman and T. Rudelius, Evidence for C-theorems in 6D SCFTs, JHEP 09 (2015) 218 [arXiv: 1506.06753] [INSPIRE].

[19] C. Cordova, T.T. Dumitrescu and K. Intriligator, Anomalies, renormalization group flows and the a-theorem in six-dimensional $(1,0)$ theories, JHEP 10 (2016) 080 [arXiv: 1506.03807] [INSPIRE].

[20] N. Bobev, M. Bullimore and H.-C. Kim, Supersymmetric Casimir energy and the anomaly polynomial, JHEP 09 (2015) 142 [arXiv:1507.08553] [INSPIRE].

[21] K. Ohmori, H. Shimizu, Y. Tachikawa and K. Yonekura, $6 d N=(1,0)$ theories on $T^{2}$ and class $S$ theories: part I, JHEP 07 (2015) 014 [arXiv: 1503.06217] [INSPIRE].

[22] M. Del Zotto, C. Vafa and D. Xie, Geometric engineering, mirror symmetry and $6 d_{(1,0)} \rightarrow 4 d_{(N=2)}$, JHEP 11 (2015) 123 [arXiv:1504.08348] [INSPIRE].

[23] K. Ohmori, H. Shimizu, Y. Tachikawa and K. Yonekura, $6 d N=(1,0)$ theories on $S^{1} / T^{2}$ and class $S$ theories: part II, JHEP 12 (2015) 131 [arXiv:1508.00915] [INSPIRE].

[24] D. Gaiotto and S.S. Razamat, $N=1$ theories of class $S_{k}$, JHEP 07 (2015) 073 [arXiv: 1503.05159] [INSPIRE].

[25] S. Franco, H. Hayashi and A. Uranga, Charting class $S_{k}$ territory, Phys. Rev. D 92 (2015) 045004 [arXiv: 1504.05988] [INSPIRE].

[26] A. Hanany and K. Maruyoshi, Chiral theories of class S, JHEP 12 (2015) 080 [arXiv: 1505.05053] [INSPIRE].

[27] H. Hayashi, S.-S. Kim, K. Lee, M. Taki and F. Yagi, A new $5 d$ description of $6 d$ D-type minimal conformal matter, JHEP 08 (2015) 097 [arXiv:1505.04439] [INSPIRE].

[28] I. Brunner and A. Karch, Branes at orbifolds versus Hanany Witten in six-dimensions, JHEP 03 (1998) 003 [hep-th/9712143] [INSPIRE].

[29] A. Hanany and A. Zaffaroni, Branes and six-dimensional supersymmetric theories, Nucl. Phys. B 529 (1998) 180 [hep-th/9712145] [INSPIRE].

[30] S.-S. Kim, M. Taki and F. Yagi, Tao probing the end of the world, PTEP 2015 (2015) 083B02 [arXiv: 1504.03672] [INSPIRE].

[31] K. Yonekura, Instanton operators and symmetry enhancement in 5 d supersymmetric quiver gauge theories, JHEP 07 (2015) 167 [arXiv:1505.04743] [INSPIRE].

[32] Y. Tachikawa, Instanton operators and symmetry enhancement in 5d supersymmetric gauge theories, PTEP 2015 (2015) 043B06 [arXiv:1501.01031] [INSPIRE].

[33] G. Zafrir, Instanton operators and symmetry enhancement in $5 d$ supersymmetric USp, SO and exceptional gauge theories, JHEP 07 (2015) 087 [arXiv: 1503.08136] [INSPIRE].

[34] D. Gaiotto and H.-C. Kim, Duality walls and defects in $5 d N=1$ theories, JHEP 01 (2017) 019 [arXiv: 1506.03871] [INSPIRE]. 
[35] A. Sen, F theory and orientifolds, Nucl. Phys. B 475 (1996) 562 [hep-th/9605150] [INSPIRE].

[36] G. Zafrir, Brane webs, 5d gauge theories and 6d $N=(1,0)$ SCFT's, JHEP 12 (2015) 157 [arXiv: 1509.02016] [INSPIRE].

[37] O. DeWolfe, A. Hanany, A. Iqbal and E. Katz, Five-branes, seven-branes and five-dimensional $E_{n}$ field theories, JHEP 03 (1999) 006 [hep-th/9902179] [INSPIRE].

[38] K.A. Intriligator, D.R. Morrison and N. Seiberg, Five-dimensional supersymmetric gauge theories and degenerations of Calabi-Yau spaces, Nucl. Phys. B 497 (1997) 56 [hep-th/9702198] [INSPIRE].

[39] O. Bergman and G. Zafrir, 5d fixed points from brane webs and O7-planes, JHEP 12 (2015) 163 [arXiv: 1507.03860] [INSPIRE].

[40] A. Hanany and E. Witten, Type IIB superstrings, BPS monopoles and three-dimensional gauge dynamics, Nucl. Phys. B 492 (1997) 152 [hep-th/9611230] [INSPIRE].

[41] S.-S. Kim and F. Yagi, $5 d E_{n}$ Seiberg-Witten curve via toric-like diagram, JHEP 06 (2015) 082 [arXiv: 1411.7903] [INSPIRE].

[42] F. Benini, S. Benvenuti and Y. Tachikawa, Webs of five-branes and $N=2$ superconformal field theories, JHEP 09 (2009) 052 [arXiv:0906.0359] [INSPIRE].

[43] H. Hayashi, H.-C. Kim and T. Nishinaka, Topological strings and $5 d T_{N}$ partition functions, JHEP 06 (2014) 014 [arXiv: 1310.3854] [INSPIRE].

[44] M. Aganagic, N. Haouzi and S. Shakirov, $A_{n}$-triality, arXiv:1403.3657 [INSPIRE].

[45] O. Bergman and G. Zafrir, Lifting 4d dualities to 5d, JHEP 04 (2015) 141 [arXiv: 1410.2806] [INSPIRE].

[46] H. Hayashi, Y. Tachikawa and K. Yonekura, Mass-deformed $T_{N}$ as a linear quiver, JHEP 02 (2015) 089 [arXiv: 1410.6868] [INSPIRE].

[47] N. Seiberg, Five-dimensional SUSY field theories, nontrivial fixed points and string dynamics, Phys. Lett. B 388 (1996) 753 [hep-th/9608111] [INSPIRE]. 